\title{
Structure formation and dynamics in molecularly thin smectic liquid crystal films
}

\author{
Dissertation \\ zur Erlangung des mathematisch-naturwissenschaftlichen Doktorgrades \\ "Doctor rerum naturalium" \\ der Georg-August-Universität Göttingen \\ im Promotionsprogramm ProPhys \\ der Georg-August University School of Science (GAUSS)
}

vorgelegt von

Benjamin Schulz

aus Karl-Marx-Stadt, Deutschland

Göttingen, 2013 
Betreuungsausschuss:

Prof. Dr. Jörg Enderlein, Drittes Physikalisches Institut, Universität Göttingen

Prof. Dr. Stephan Herminghaus, Dynamik komplexer Fluide, Max-Planck-Institut für Dynamik und Selbstorganisation, Göttingen

\section{Mitglieder der Prüfungskommission}

Referent : Prof. Dr. Jörg Enderlein, Drittes Physikalisches Institut, Georg-August-Universität Göttingen

Koreferent : Prof. Dr. Stephan Herminghaus, Dynamik komplexer Fluide, Max-Planck-Institut für Dynamik und Selbstorganisation, Göttingen

Weitere Mitglieder der Prüfungskommission:

PD Dr. Christian Bahr, Dynamik komplexer Fluide, Max-Planck-Institut für Dynamik und Selbstorganisation, Göttingen

Prof. Dr. Cynthia A. Volkert, Institut für Materialphysik, Georg-August-Universität Göttingen

Dr. Iwan Schaap, Drittes Physikalisches Institut, Georg-August-Universität Göttingen

Dr. Jean-Christophe Baret, Tröpfchen, Membranen und Grenzflächen, Max-Planck-Institut für Dynamik und Selbstorganisation, Göttingen

Tag der mündlichen Prüfung: 29.04.2013 


\section{Abstract}

This doctoral thesis presents a study of thin films of smectic liquid crystals. This class of material shows the property to form stable films of only a few molecules thickness both on substrates and freely suspended in air. This feature together with the anisotropy of the material offers the possibility to prepare and investigate samples that cannot be formed by other materials. As the dimensions of the films are reduced, the physical properties are expected to change with respect to the observed surface structures and the dynamics in the film.

For freely suspended films, the presented work analyses the translational dynamics in films with a thicknesses ranging from 22 down to only 2 smectic layers, using single molecule tracking. For all the studied compounds it was observed that thinner films show a faster diffusion. The measured effect is quantitatively larger than predicted from theory. However, molecular dynamics simulations support the finding of drastic changes for ultrathin films. Within the smectic A phase (i.e. not close to phase transitions), the temperature dependence is observed to show the classical Arrhenius behaviour. Contrary to that, in the vicinity of the phase transition to a smectic phase with in-plane ordering, the diffusion coefficient scales similarly to the one of a glass transition. Also heterogeneous free-standing film are studied concerning the molecular behaviour. It is shown that the molecules have a strong tendency to avoid crossing phase boundaries.

For liquid crystal films on substrates, this work presents thin films of $8 \mathrm{CB}$ on silicon wafers. Despite the numerous studies of this material-substrate combination, no controlled creation of films with a small number of layers was reported so far. Such homogeneous films are created and studied with respect their inner structure using atomic force microscopy and ellipsometry. The dynamics is analysed using single molecule tracking, showing a slowdown of the diffusion when going to thinner films. These homogeneous films can also be used to create droplets with a volume below $10^{-14}$ litres, which in turn can be used to study smectic layering on the surface with the main volume of the droplet in the nematic phase. It is also shown how the homogeneous films can be employed as a base material for the writing of stable structures on the nanoscale. 


\section{Kurzzusammenfassung}

Diese Doktorarbeit beschäftigt sich mit der Untersuchung von dünnen Filmen smektischer Flüssigkristalle. Diese Materialklasse besitzt die Eigenschaft, dünne Filme mit nur wenigen Molekülen Dicke sowohl auf Substrat als auch freistehend in Luft auszubilden. Zusammen mit der Anisotropie des Materials ermöglicht dies die Herstellung und Untersuchung von Proben, welche nicht aus anderen Materialien hergestellt werden können. Wenn man die Abmessungen der Filme reduziert, steht zu erwarten, dass sich die physikalischen Eigenschaften hinsichtlich der beobachteten Oberflächenstrukturen und der Dynamik innerhalb des Films ändern.

Für freistehende Filme untersucht die vorliegende Arbeit die Translationsdynamik in Filmen mit einer Dicke zwischen 22 und nur noch 2 smektischen Schichten mit Hilfe von Einzelmoleküldetektion. Für alle untersuchten Stoffe wurde hierbei eine schnellere Diffusion für dünnere Filme beobachtet. Der gemessene Effekt ist dabei größer als von der Theorie vorhergesagt. Jedoch unterstützen molekulardynamische Simulationen die gefundenen starken Änderungen für ultradünne Filme. Innerhalb der smektischen A Phase (d. h. nicht in der Nähe von Phasenübergängen), folgt die beobachtete Temperaturabhängigkeit der Diffusion dem klassischen Arrhenius-Verhalten. Im Gegensatz dazu skaliert in der Nähe von Phasenübergängen zu Phasen mit Positionsordnung innerhalb der Schichten der Diffusionskoeffizient ähnlich wie bei einem Glasübergang. Außerdem wurden heterogene Filme hinsichtlich ihres molekularen Verhaltens untersucht. Es wurde gezeigt, dass die Moleküle eine starke Tendenz haben, das Überqueren von Domänengrenzen zu vermeiden.

Für Flüssigkristallfilme auf Substrat untersucht die Arbeit dünne 8CB-Filme auch Siliziumwafern. Trotz der zahlreichen Studien dieser Material-Substrat-Kombination wurde bisher noch keine kontrollierte Herstellung von Filmen mit einer kleinen Anzahl smektischer Schichten beschrieben. Solche homogenen Filme wurden hergestellt und bezüglich ihrer inneren Struktur mit Hilfe von Rasterkraftmikroskopie und Ellipsometrie untersucht. Die Dynamik wurde mittels Einzelmoleküldetektion gemessen und eine Verlangsamung der Bewegung für dünnere Filme festgestellt. Die homogenen Filme können auch verwendet werden, um Tröpfchen mit Volumina geringer als $10^{-14}$ Liter herzustellen, welche wiederum verwendet werden können um die Ausbildung smektischer Schichten nahe der Oberfläche zu beobachten, während sich der Rest des Tropfens in der nematischen Phase befindet. Es wurde auch gezeigt, wie die homogenen Filme als Ausgangsmaterial für das Schreiben von stabilen Strukturen im Nanometermaßstab genutzt werden können. 


\section{Contents}

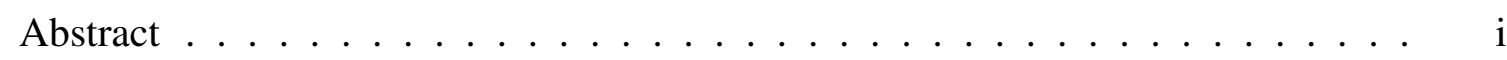

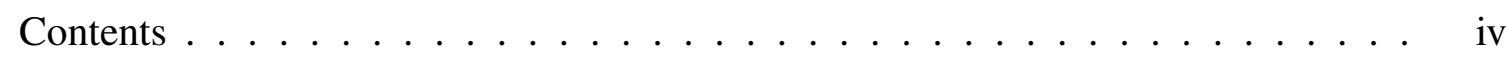

List of Figures . . . . . . . . . . . . . . . . . . . . . vii

List of Tables ........................... . . ix

\begin{tabular}{lll}
\hline & Introduction & 1
\end{tabular}

2 Liquid crystals 5

2.1 General aspects . . . . . . . . . . . . . . . . . . . . 5

2.2 Classification of liquid crystals $\ldots \ldots \ldots \ldots \ldots$

2.3 Deformation of liquid crystals $\ldots \ldots \ldots \ldots \ldots$. . . . . . . . . . . . . . . . . . . .

2.4 Phase transitions in liquid crystals . . . . . . . . . . . . . . . 12

$2.4 .1 \quad$ Maier-Saupe theory . . . . . . . . . . . . . . . . 13

2.4 .2 Ginzburg-Landau theory . . . . . . . . . . . . . . . 16

2.5 Thin smectic films . . . . . . . . . . . . . . . . . . . . . . . . . . . . . . . . . . 19

2.5 .1 Smectic films on substrates . . . . . . . . . . . . . . . . 19

2.5 .2 Free-standing smectic films . . . . . . . . . . . 21

3 Diffusion analysis using single molecule tracking 25

3.1 Fundamentals of diffusion . . . . . . . . . . . . . . 25

3.2 Diffusion in liquid crystals . . . . . . . . . . . . . . . . 27

$3.3 \quad$ Fluorescent probe molecules for diffusion analysis . . . . . . . . . . . . . . 29

3.3 .1 Fluorescence imaging . . . . . . . . . . . . . . 29

3.3 .2 Single molecule tracking . . . . . . . . . . . . . 31

4 Methods and experimental techniques $\quad 35$

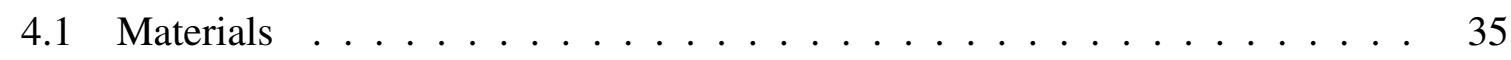

4.2 Sample preparation $\ldots \ldots \ldots \ldots \ldots \ldots$ 
4.3 Atomic force microscopy $\ldots \ldots \ldots \ldots \ldots \ldots$. . . . . . . . . . . . 41

4.4 Ellipsometry . . . . . . . . . . . . . . . . . . . . . 42

4.5 Single molecule setup . . . . . . . . . . . . . . . . . 44

5 Diffusion in free-standing liquid crystal films 49

5.1 Faster diffusion in thinner films . . . . . . . . . . . . . . . . 52

5.1.1 Experimental results for different compounds in the smectic A phase. 52

\begin{tabular}{|lll}
5.1 .2 & Theoretical considerations of the diffusion in thin free-standing films & 60
\end{tabular}

5.1 .3 Simulation of the self-diffusion in free-standing films . . . . . . . 63

5.2 Diffusion in proximity to phase transitions . . . . . . . . . . . . . 64

$5.2 .1 \quad$ Temperature dependence within the smectic A phase of 8CB . . . . . 64

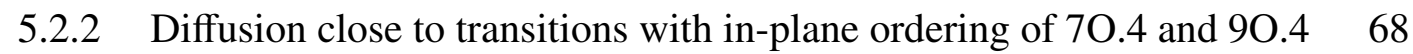

5.2 .3 First order transition from smectic A to smectic $\mathrm{C}$ of $\mathrm{C} 7$. . . . . . 74

5.3 Introduction of zero-shear viscosity in free-standing films . . . . . . . . . 76

5.4 Diffusion in heterogeneous free-standing films . . . . . . . . . . . . . 77

$5.4 .1 \quad$ Laterally inhomogeneous films . . . . . . . . . . . . . . . . . . . . . 78

5.4 .2 Axially inhomogeneous films . . . . . . . . . . . 80

6 Ultrathin films of 8CB on silicon substrates 85

6.1 Creation and properties of ultrathin smectic films on silicon substrates . . . . 85

$6.1 .1 \quad$ AFM measurements of the surface structure . . . . . . . . . . . 85

6.1 .2 Ellipsometry measurements of the film thickness . . . . . . . . . . 94

6.2 Spreading of femtolitre droplets . . . . . . . . . . . . . . 97

6.3 Single molecule diffusion in thin films on substrates . . . . . . . . . . . 100

6.4 Writing of soft structures on the nanoscale . . . . . . . . . . . . . . . 104

\begin{tabular}{lll}
\hline 7 & Summary and outlook & 115
\end{tabular}

\begin{tabular}{ll}
\hline Appendix & 119
\end{tabular}

\begin{tabular}{ll}
\hline References & 121
\end{tabular} 


\section{List of Figures}

$2.1 \quad$ Structure of the nematic and cholesteric phase . . . . . . . . . . 7

2.2 Schlieren textures . . . . . . . . . . . . . . . . . 8

2.3 Structure of the smectic A and smectic $\mathrm{C}$ phase . . . . . . . . . . . . 9

2.4 Structure of the smectic $\mathrm{F}$ and smectic $\mathrm{G}$ phase $\ldots \ldots \ldots$. . . . . . . . 10

2.5 Expected X-ray diffraction patterns for smectic phases $\ldots \ldots \ldots$

$2.6 \quad$ Fundamental deformations of liquid crystals . . . . . . . . . . . . . 11

$2.7 \quad$ Temperature dependence of the order parameter . . . . . . . . . . . . . 16

2.8 Focal conic domains and hemicylinders on solid substrates . . . . . . . . 20

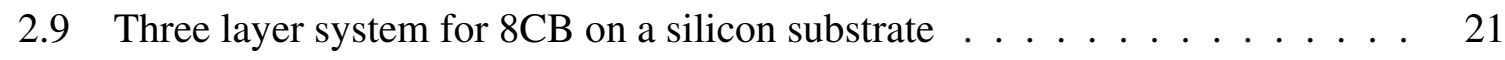

2.10 Sketch of a free-standing film . . . . . . . . . . . . . . 21

2.11 Free-standing films with hexatic phase in the outer regions and smectic phase in the middle . . . . . . . . . . . . . . . . 23

2.12 Layer-by-layer thinning of overheated free-standing films . . . . . . . . . . . 24

3.1 Jablonski diagram $\ldots \ldots \ldots \ldots \ldots \ldots \ldots$

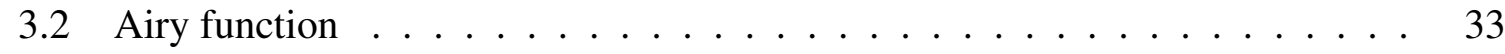

3.3 Statistical error in calculated mean squared displacements . . . . . . . . . . 34

4.1 Molecular structures of the employed mesogens $\ldots \ldots \ldots$. . . . . . . . . 36

4.2 Molecular structure of Nile red . . . . . . . . . . . . . . . . . . . . . . 38

4.3 Schematic top view and photo of the sample holder for the free-standing films 40

4.4 Schematic setup of an atomic force microscope . . . . . . . . . . . . . 42

4.5 Measurement concept of ellipsometry . . . . . . . . . . . . . . . . . . 43

4.6 Schematic representation of the single molecule microscope . . . . . . . 45

4.7 Photo of the single molecule microscope . . . . . . . . . . . . . . 46

4.8 Spectrum of the white light source . . . . . . . . . . . . . . . . 47

$4.9 \quad$ Examples of accepted and rejected trajectories $\ldots \ldots \ldots$. . . . . . . . . 47 
5.1 Determination of the film thickness $\ldots \ldots \ldots \ldots$. . . . . . . . . . 51

5.2 Comparison of different methods of calculating the diffusion coefficient . . . 52

5.3 Film thickness dependence of diffusion in thin free-standing films of $8 \mathrm{CB}$. . 54

5.4 Film thickness dependence of diffusion in thin free-standing films of pyrimi-

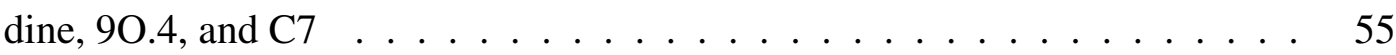

$5.5 \quad$ Film thickness dependence of the scaled diffusion coefficients $\ldots \ldots . .57$

5.6 Standard deviation of the diffusion coefficients of $8 \mathrm{CB} \ldots \ldots \ldots \ldots$

$\begin{array}{lll}5.7 & \text { Distribution of squared displacements for a film of two and ten layers of } 8 \mathrm{CB} & 59\end{array}$

5.8 Definition of the values for the calculation of the increase in diffusion . . . 60

5.9 Theoretical curve describing the increase at a perfectly slipping surface . . . 62

5.10 Simulated values for the film thickness dependence of the diffusion coefficients 64

5.11 Temperature dependence of diffusion for a seven layer film $\ldots \ldots \ldots 66$

5.12 Arrhenius plots for three to seven layers of $8 \mathrm{CB} \ldots \ldots \ldots \ldots \ldots$

5.13 Temperature dependence of the diffusion coefficient of in a 10 layer film of 70.4 68

5.14 Temperature dependence of the diffusion coefficient of in a 3 and a 6 layer film of $70.4 \ldots \ldots \ldots \ldots$

5.15 Extrapolated bulk values of the diffusion in $8 \mathrm{CB} \ldots \ldots \ldots \ldots$

5.16 Temperature dependence of the diffusion coefficient in 90.4 measured over 15

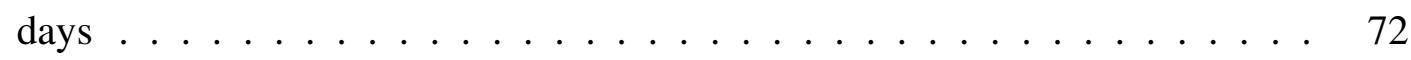

5.17 Arrhenius plot for the diffusion of $90.4 \ldots \ldots \ldots \ldots \ldots$

5.18 Change of the phase transition temperature of $90.4 \ldots \ldots \ldots \ldots$

5.19 Diffusion coefficients around a first order transition from smectic $\mathrm{C}$ to smectic $\mathrm{A} 75$

5.20 Temperature-dependence of the zero-shear viscosity in an 8CB film consisting of seven layers $\ldots \ldots \ldots \ldots \ldots$. . . . . . . . . . . . . 77

5.21 Image of a laterally inhomogeneous film in the fluorescence microscope . . . 78

5.22 Schematic cross section of a laterally inhomogeneous film $\ldots \ldots \ldots . .79$

5.23 Trajectories of two molecules diffusing only within the smectic A phase . . . 80

5.24 Diffusion coefficients and step length probabilities for a laterally inhomogeneous film . . . . . . . . . . . . . . . . . . . . . . 81

5.25 Layer dependent diffusion in a film close to a hexatic phase transition $\ldots . .81$

5.26 Examples for trajectories of slow and fast molecules in an equilibrium film. . 82

5.27 Step length distribution for an axially inhomogeneous film . . . . . . . 83

$6.1 \quad$ Examples for surface structures of $8 \mathrm{CB} \quad \ldots \ldots \ldots \ldots \ldots \ldots$

6.2 Process of creation of an additional layer $\ldots \ldots \ldots \ldots \ldots \ldots$ 
6.3 Examples for binarised images $\ldots \ldots \ldots$. . . . . . . . . . . . 89

6.4 Number of deposited layers depending on the 8CB concentration . . . . . . . 90

6.5 Cross-section through a porous film . . . . . . . . . . . . . . 91

6.6 Phase difference between different molecular layers . . . . . . . . . . . . . 92

6.7 Changes in the surface structure after 5 days $\ldots \ldots \ldots$. . . . . . . . . . . . . . . . . . 93

6.8 Ellipticity coefficients for completed layers $\ldots \ldots \ldots$. . . . . . . . 95

6.9 Calculated theoretical ellipticity coefficients . . . . . . . . . . . . . 96

6.10 Comparison of measured film thicknesses with AFM and ellipsometry . . . . 97

6.11 Spreading of smectic droplets . . . . . . . . . . . . . . . . . . . . . . 98

6.12 Cooling nematic droplets reveals smectic wetting . . . . . . . . . . . . 99

6.13 Layer-dependent phase transition temperature . . . . . . . . . . . . . 100

6.14 Comparison of silicon substrates with native oxide layer and $100 \mathrm{~nm}$ oxide. . 101

6.15 Diffusion in thin $8 \mathrm{CB}$ films on substrates . . . . . . . . . . . . . . 103

6.16 Distribution of diffusion coefficients for a 5 layer film . . . . . . . . . . . . 104

6.17 Filling of selected pores of a film . . . . . . . . . . . . . 105

6.18 Creation of an isolated structure of one layer thickness $\ldots . . . . . .106$

6.19 Writing of a single line with a width of around $100 \mathrm{~nm}$. . . . . . . . . 107

6.20 Writing of several layers on top of each other . . . . . . . . . . . . 108

6.21 Combined filling of pores and writing of a new layer . . . . . . . . . . . 109

6.22 Sketch for the suggested mechanism of AFM writing . . . . . . . . . . . 110

6.23 Examples for more than one smectic layer written during one scan . . . . . . 112

6.24 Variable phase properties of the first written layer . . . . . . . . . . . 113 



\section{List of Tables}

$4.1 \quad$ Phase transition temperatures of the used mesogens $\ldots \ldots \ldots . \ldots 37$

5.1 Scaling coefficients of the film thickness dependence of diffusion for 4 differ-

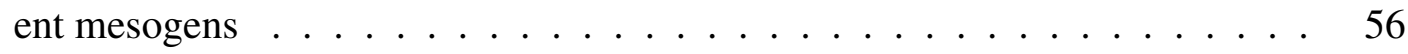

5.2 Decay constants of the Arrhenius fits for three to seven layers of 8CB . . . . 67

5.3 Diffusion coefficients $D_{\text {hex }}$ and $D_{\text {smA }}$ for a laterally inhomogeneous film . . . 80 



\section{Chapter 1}

\section{Introduction}

Measuring the properties of materials at surfaces and interfaces has evolved to an independent field of research in times where miniaturisation is considered as common sense. For liquid crystals - the material studied in this doctoral thesis - this particularly holds true because the molecular interactions influence the properties of the material over length scales of micrometres. The aim of the presented work is to analyse dynamic and structural properties of liquid crystals at either free or solid surfaces by reducing the film thickness to the experimental limits, thereby eliminating the bulk properties.

When studying thin films of only some nanometres thickness, the focus of the probed properties cannot be described in terms of continuum mechanics but rather in terms of the interactions at the molecular level, leading to properties that can be very different from those of the bulk material. Thin films of semiconductors, for example, show drastic changes in the conductivity of the material. When focusing on liquid materials, however, the creation of a stable thin film is not straightforward as the possibility to freely move the molecules results in an energy minimum in dewetting instead of forming a homogeneous film for most of the cases. Using smectic liquid crystal materials enables the experimentalist to create thin films of different kinds: The material forms stable films when freely suspended in air [1], on solid substrates [2], and on liquid surfaces [3]. In this thesis we will focus on the first two cases.

The class of materials called liquid crystals has the feature of, despite being a liquid material, exhibiting ordering properties with at least one preferred direction. Owing to the resulting anisotropic nature, this gives rise to the formation of self-organised structures. Also the dynamic behaviour inevitably has to differ from the one of isotropic liquids. By preparing such materials as thin films, one adds another constraint in form of a one-dimensional geometrical 
confinement, which in turn induces external stress to the system. This leads to a complex energy minimisation in the material.

Due to the tendency of liquid crystal molecules to orient along a common direction, the material shows elastic behaviour. Therefore liquid crystals can compensate external stresses. One of the consequences of this property is the possibility to form free-standing films. This term describes films that are stable without support and show a perfectly homogeneous film thickness over macroscopic areas. The film thickness can be reduced down to only two molecular layers, thereby creating homogeneous samples with aspect ratios of width to thickness of the order of one million. The movement of the molecules in the film is therefore quasi twodimensional. Although these films are known for long, the properties of the translational dynamics at the molecular level remain widely unexplored. The presented work addresses this open question and aims to analyse to which extent the reduced dimensions of the film influence the behaviour of the in-plane diffusion.

The studies of thin films of liquid crystals on various substrates are abundant. Even restricting ourselves to the case of the common liquid crystal compound $8 \mathrm{CB}$ - that is used also for our experiments on substrates - there is a multitude of different experimental methods applied to study mainly the structure formation. Studies were done using optical microscopy [4], atomic force microscopy [5], X-ray scattering [6], Raman scattering [7], or fluorescence polarised microscopy [8]. The self-organised structures formed on the surface of smectic liquid crystals are considered to have possible application for photonic patterning [9] or superhydrophobic surface [10]. However, the controlled creation of 8CB films with an arbitrary thickness in the range of only a few molecular layers was not achieved so far. The creation and analysis of such films is the second main topic of this work.

The thesis is subdivided into seven chapters. Chapter 2 introduces the fundamental properties of liquid crystals. The focus will be put on the one hand on the presentation of the different phases that are dealt with in the course of this work. On the other hand the ability of certain kinds of liquid crystals to form molecularly thin film will be presented. The particular properties that arise in such samples are explained.

This is followed by a chapter about the fundamentals of diffusion. In the case of liquid crystals, the shape anisotropy of the molecules also induces an anisotropy of the diffusion with different properties along the long axis of the molecules and perpendicular to it. This 
part also presents the method of single molecule tracking that was used for the analysis of the diffusion.

In chapter 4, the used materials and the experimental methods are presented. The employed liquid crystals are introduced with their structure and phase sequence. The used setups for atomic force microscopy, ellipsometry, and single molecule tracking are explained.

The study of the analysis of thin free-standing films is presented in chapter 5 . The first section deals with the behaviour observed upon reducing the thickness to the experimental limit of two layers. Hereafter, the focus is put on different kinds of phases transitions. Depending on the material, there is a variety of transitions possible that in turn induce distinct properties in the vicinity of the transition. The chapter also shows results on the molecular behaviour of individual molecules in inhomogeneous films.

The results on $8 \mathrm{CB}$ films on silicon substrates are presented in chapter 6. This includes the creation as well as the analysis of the structure and the dynamics present in the films. The thesis concludes with the introduction of a method for nanolithography of soft matter based on the thin $8 \mathrm{CB}$ films.

Finally, chapter 7 gives a summary of the presented results and shows the questions that the presented work has to leave open or that are raised by its results. 



\section{Chapter 2}

\section{Liquid crystals}

In this chapter the main properties of liquid crystals will be explained and the features that characterise different types of liquid crystals are described. After the definition of the different phases, the focus will be put on smectic liquid crystals and their ability to form ultrathin films with or without substrates, which is the main topic of this work. As a lot of experiments are conducted close to the phase transition between different phases, the basic theoretical description of phase transitions will be given. Finally the two fundamentally different ways of preparing liquid crystalline films with a thickness of only a few molecules will be shown.

\subsection{General aspects}

The term liquid crystals (LCs) summarises a group of materials that cannot be classified in terms of the three classical states of solid, liquid, and gas but combine properties of liquids and solids. On the one hand, a characteristic liquid feature exhibited by all LCs is that the material is flowing which means the easy adaptation of an arbitrary shape. On the other hand all LCs show higher ordering than an isotropic liquid which leads to properties like birefringence that traditionally is a typical feature of anisotropic solid materials.

Apart from this common feature, there are additional properties that are only shown by some of the materials and therefore give rise to further classifications. The most fundamental one is the distinction between lyotropic and thermotropic LCs. Lyotropics are solutions of amphiphilic molecules in a solvent in which the molecules self-organise in hierarchical structures like double-layers or micelles [11]. Here the parameters that govern the material properties are the concentration of the amphiphilic molecules and the temperature. Thermotropics on 
the contrary are pure materials that show ordering effects without additional solvent and that have the temperature as sole controle parameter. The molecules that constitute a thermotropic LC phase are called as mesogens. In this work, only thermotropic LCs are considered and henceforth would refer to them unless otherwise stated.

The reason for the ordering in thermotropic LCs is the shape anisotropy of the molecules themselves. There are different possibilities of shape anisotropy like the most common rodlike shape, disk shaped [12] (called discotic), or V-shaped molecules [13]. The driving force for the mesoscopic ordering of the molecules can be seen in the fact that the orientation of the molecules in the same direction minimises the average distance between the molecules and thus minimises the potential energy of the entire system. The direction along which the molecules orient is called the director $\vec{n}$. However, contrary to solid materials, the thermal energy is still big enough to conserve the mobility of the molecules while they are keeping their average orientation.

\subsection{Classification of liquid crystals}

The simplest LC phase is the nematic phase where the molecules exhibit the already mentioned orientation property as the sole ordering feature as shown in figure 2.1. The average center of mass is randomly distributed in this phase, apart from the short range order that is also known for all isotropic liquids. The orientation of the molecules is the reason why liquid crystal materials are so easy to identify as this leads to their birefringence. The polarisability of the molecules is different along the long and the short axis of the molecules, which in turn leads to different refractive indices in the direction of $\vec{n}$ or perpendicular to it characteristic of a birefringent material.

The typical length scales on which the orientation is conserved are mesoscopic. When a thin nematic film is observed between crossed polarizers, the birefringence causes a characteristic texture (so called schlieren texture) as shown in figure 2.2. Generally all the phases of liquid crystals in principle can be distinguished to a big extent only by the textures observable using polarising microscopy (numerous examples given in [14]).

Locally similar to the nematic phase is the cholesteric phase (also called chiral nematic phase), which is formed in case of chiral mesogens or if chiral molecules are added to a nematic phase. The presence of chirality leads to a twist of the director around an axis of rotation, as shown in figure 2.1 . The length over which the director rotates by $360^{\circ}$ is called the 


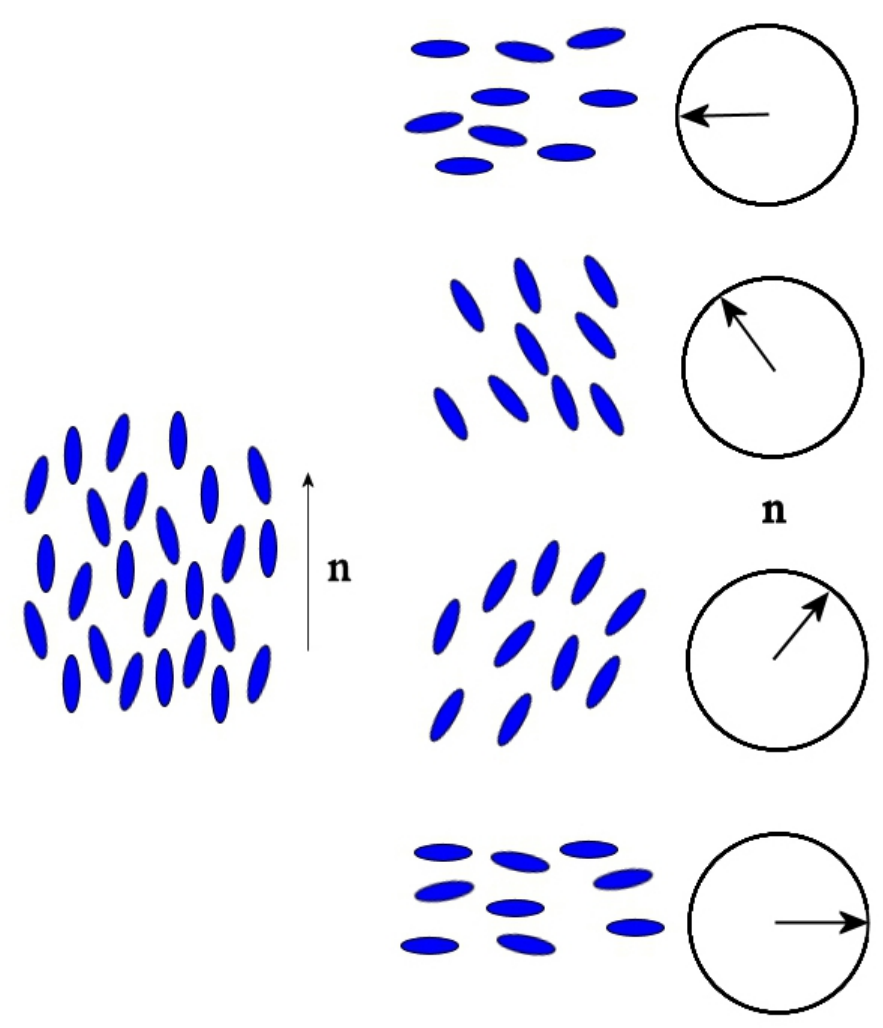

Figure 2.1: Schematic molecular orientation in the nematic (left) and cholesteric (right) phase. The nematic phase has only a common average orientation along the director $\vec{n}$ as a defining feature. In the cholesteric phase, the director is twisted if compared in successive cross sections of the material. The drawn planes have no physical meaning but are only shown for understanding. The images represent regions with a distance of half the pitch between top and bottom image.

pitch $p$. However, because of the inversion symmetry of $\vec{n}$, the original molecular orientation is already reached after $180^{\circ}$ (compare figure 2.1). The reason why the cholesteric phase is locally very similar to the nematic phase is that the pitch is of the order of hundreds of nanometers [15], which means that it is a lot bigger than the molecular dimensions.

In order to characterise the strength of the orientation, it is desirable to define an order parameter $S$ as a quantitative measure. Due to the inversion symmetry of LCs, the average orientation with respect to the director $\langle\theta\rangle$ (with $\theta$ being the angle between the actual molecular orientation and $\vec{n}$ ) is not an appropriate choice as it is always zero. Although there would be several ways to define a reasonable order parameter, the consensus in literature is to use the average of the second Legendre polynomial of the cosine of $\theta$ : 


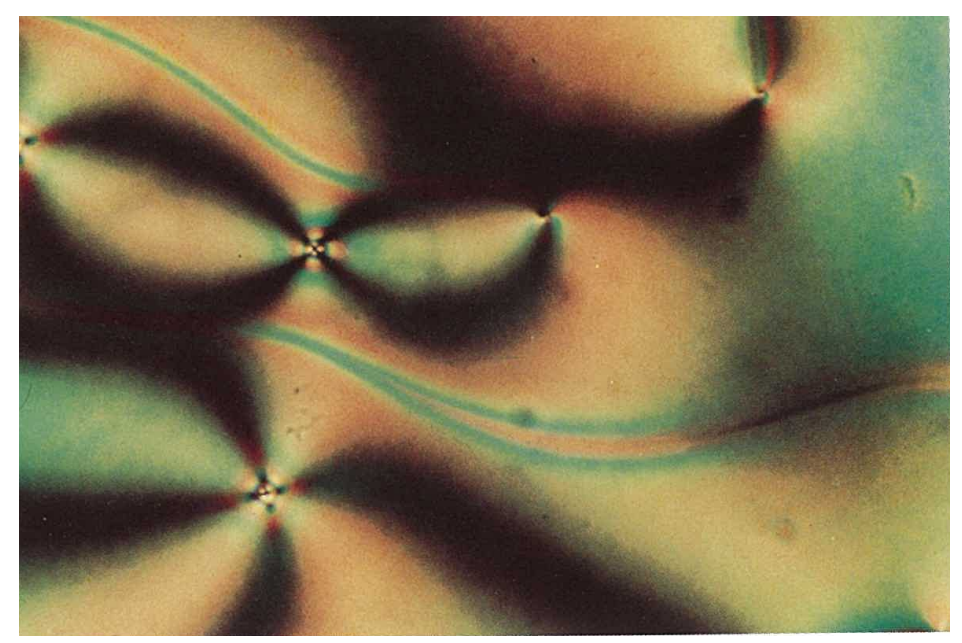

Figure 2.2: Characteristic schlieren textures of a thin nematic liquid crystal film observed between crossed polarizers (image taken from [14]).

$$
S=\frac{1}{2}\left\langle 3 \cos ^{2} \theta-1\right\rangle
$$

This definition has the advantage that it is normalised, i.e. for a perfect order with no fluctuations around $\vec{n}(\theta \equiv 0), S$ would be 1 , whereas in the case of isotropic behaviour the order parameter would be 0 . In principle, $S$ can also be negative but this is unphysical as it would mean mainly an orientation perpendicular to the preferred direction $\vec{n}$ which is an oxymoron. As we will see in section 2.4.1, there is a very general dependence of $S$ on the temperature for all nematic materials.

Another ordering feature that can be observed in some of the LCs is a one-dimensional positional ordering, which means the arrangement of the molecules in equidistant layers. As such a phase also shows mobility on the molecular level, one should more think of a higher probability of finding the center of mass at a certain position than real layering. The LC phase that shows this property is called smectic phase. Here again different subphases can be distinguished depending on the presence of possible additional ordering properties and on the molecular orientation with respect to the layers. A systematic presentation of the smectic subphases can be found in [14]. In the following we will present only the phases that will appear later in this work.

The two most commonly used smectic phases are the ones without additional positional order. Within the layers the molecules behave like an ordinary liquid. The two phases differ 


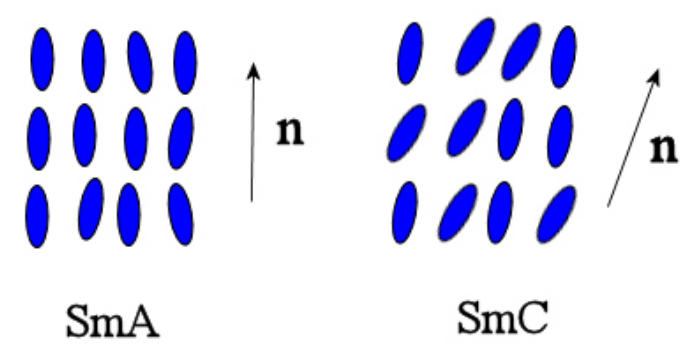

Figure 2.3: Schematic molecular arrangement in the smectic phase with the characteristic arrangement in layers. left: smectic A phase with molecular orientation perpendicular to the layers, right: smectic $\mathrm{C}$ phase with a tilt angle between $\vec{n}$ and the layer normal

in the molecular orientation with respect to the layers. In case the molecules are oriented parallelly to the layer normal the phase is called smectic A ( $\mathrm{smA})$, in case of a tilt angle between $\vec{n}$ and the layer normal the phase is called smectic C (smC) (see figure 2.3).

In some phases, the molecules tend to order more or less strictly in form of a hexagonal close packing, due to which such phases are called hexatic phases. Such materials show a sixfold long-range bond orientational order. On the contrary, the positional order is rather short-range (around $10 \mathrm{~nm}$ ) which, however, is considerably larger than in a normal smectic A phase [16]. If the molecules in such a phase are tilted in the direction of the short diagonal of the hexagon the phase is called smectic F. The structure is shown in figure 2.4. Between different layers there is no correlation in the position of the molecules.

The positional order, however, can also be long-range to form a crystalline smectic phase. In this case, there is a strong hexagonal positional order that also extends across layers. Still the molecules show considerable dynamics [14]. So as for the layering there is no rigid crystalline grid but rather a sixfold sinusoidal fluctuation of the density. For a tilt in the same way as for the smectic F phase the crystalline phase is called smectic G.

The introduced smectic phases can be distinguished most easily by their X-ray diffraction or electron diffraction patterns as shown in figure 2.5. For the smectic A phase there is no in-plane ordering at all. Consequently, there is only a diffraction ring indicative of random orientation within the layers. For hexatic phases there is a six-fold symmetry observed with smeared out maxima. For the crystalline phases, finally, one finds discrete diffraction peaks showing a strong ordering. 


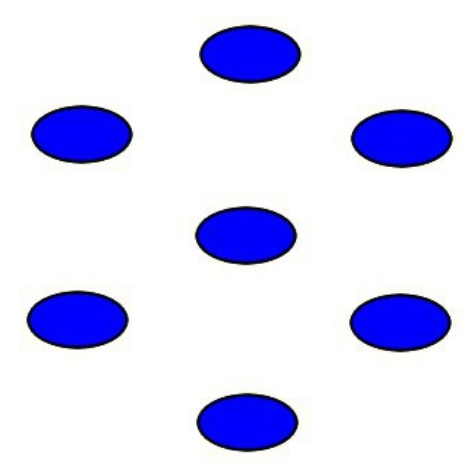

Figure 2.4: Schematic top view of a layer for phases with hexagonal in-plane ordering. Both for the smectic $\mathrm{F}$ and the smectic $\mathrm{G}$ phase the molecules are tilted with respect to the layer normal. Note that the depicted ellipses do not show the full extension of the molecules but only represent the direction of tilt.

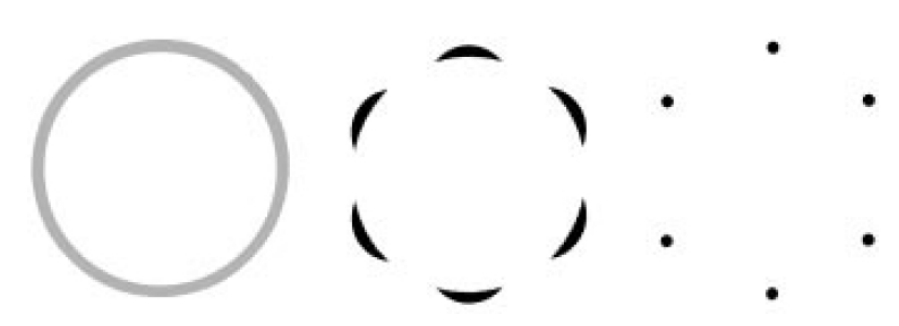

Figure 2.5: Expected X-ray diffraction patterns of smectic A (left), hexatic (middle), and crystalline (right) phases (image taken from [17]).

To characterise the ordering of a smectic material, one defines an order parameter $\Sigma$ that gives information about the strength of the orientational order and the organisation in layers. It is defined as follows [18]:

$$
\Sigma=\left\langle\cos \left(\frac{2 \pi z_{\mathrm{i}}}{d}\right) \frac{1}{2}\left(3 \cos ^{2} \theta-1\right)\right\rangle,
$$

where $z_{\mathrm{i}}$ is the position of molecule $\mathrm{i}$ and $d$ is the spacing of the smectic layers. While the second term is identical to the nematic order parameter $S$, the first term characterises the layering of the material. 

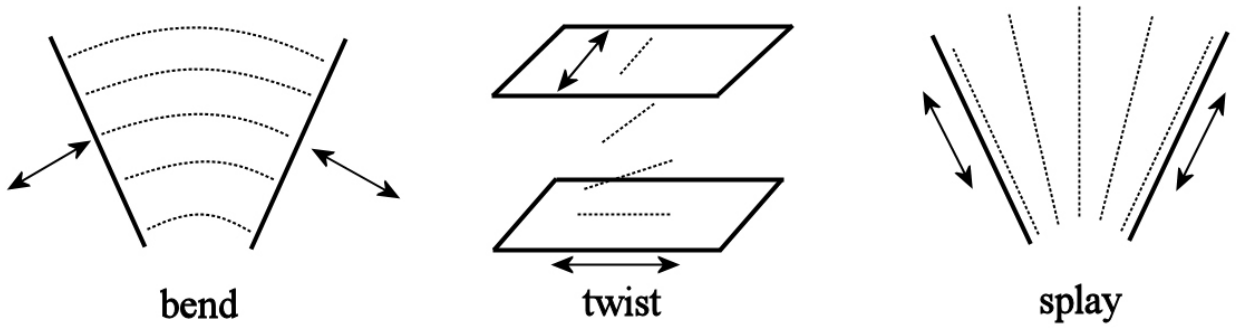

Figure 2.6: Fundamental deformations of a liquid crystal in a confining geometry. The thick solid lines show walls, the double-headed arrows anchoring on the surface, and the dotted lines the local director orientation.

\subsection{Deformation of liquid crystals}

The elastic deformation of a liquid crystal structure refers to the spatial change of the director $\vec{n}$ in a continuous manner. While in an undisturbed bulk system one finds a constant orientation of $\vec{n}$ throughout a whole domain, there might be forces that lead to a variation of the director. Possible reasons for the deformations include all forces that influence the orientation of the molecules, for example external electric or magnetic fields or spatial confinement of the material. All kinds of deformations in liquid crystals can be expressed as combinations of the three fundamental deformations that are shown in figure 2.6. All cases shall be considered for a nematic liquid crystal that is confined between plane glass plates with different kinds of anchoring on the surface. With anchoring we mean that the molecules have a preferred orientation at the solid interface, that gives a boundary condition for the director for this particular surface. There are different ways to achieve anchoring using a variety of chemical or physical treatments [2, 19]. With the help of this we can force the material into the desired conformation. The first possible deformation is the bend deformation. Here, when spatially moving in the direction of $\vec{n}$, the director is tilted to either side. We get a turning of the director with a rotation axis perpendicular to $\vec{n}$, so that the deformation energy has to increase with increasing absolute value of $(\vec{n} \times \operatorname{curl} \vec{n})$.

A twist deformation is achieved for example in the case of two parallel glass plates with uniform planar anchoring of the molecules and a twist of $90^{\circ}$ between the anchoring directions. The director orientation is twisted when moving perpendicular to it. Therefore, the deformation energy is increased with increasing absolute value of $(\vec{n} \cdot \operatorname{curl} \vec{n})$. It is worthwhile mentioning that the arrangement shown in figure 2.6 presents the geometry of a twisted nematic cell as used for the earliest LC displays. 
The twist deformation is inherent for the director of a cholesteric LC without any external force. Here the twist in the undisturbed structure is caused by the intrinsic chirality of the material.

The splay deformation, finally, is obtained for diverging lines of the director. For this reason, the divergence of the director field is non-zero and the deformation energy is increased with increasing absolute value of $(\operatorname{div} \vec{n})$.

As mentioned, the total energy of a deformed liquid crystal can be described as the sum of the contributions of all the individual terms. The fundamental deformations can have different proportionality parameter $K_{\mathrm{i}}$ (called elastic constants). Also, the energy should be proportional to the square of the curvature of the surface, so that we can write the free energy density as

$$
f_{\text {deform }}=\frac{1}{2}\left[K_{\text {bend }}(\vec{n} \times \operatorname{curl} \vec{n})^{2}+K_{\text {twist }}(\vec{n} \cdot \operatorname{curl} \vec{n})^{2}+K_{\text {splay }}(\operatorname{div} \vec{n})^{2}\right] .
$$

A simplification of the given equation is obtained under the assumption of the equality of all the elastic constants. This would lead to

$$
f_{\text {deform }} \approx \frac{K}{2}\left[(\vec{n} \times \operatorname{curl} \vec{n})^{2}+(\vec{n} \cdot \operatorname{curl} \vec{n})^{2}+(\operatorname{div} \vec{n})^{2}\right] .
$$

Also one can simplify the term for the elastic energy for the case of the smectic phase. As mentioned above, we have the condition of equidistance of the smectic layers. This condition, however, is violated by both the bend and twist deformation. Consequently, possible deformations of smectics are solely splay deformations. Thus the free energy density reduces to

$$
f_{\text {deform }}^{\text {smectic }}=\frac{K_{\text {splay }}}{2}(\operatorname{div} \vec{n})^{2} .
$$

\subsection{Phase transitions in liquid crystals}

In this section an overview of the theoretical treatment of phase transitions in LCs, which will be considered later in this work, is given. The intention is not to offer a complete analytical description, but rather to convey the ideas that are generally used to describe different kinds of phase transitions. For the complete analysis of the different transitions the detailed derivations in textbooks can be consulted [15, 20, 21]. 


\subsubsection{Maier-Saupe theory}

The first quantitative explanation of the spontaneous symmetry breaking in liquid crystals at the transition temperature from the nematic to the isotropic phase was given by Maier and Saupe in three publications in the time from 1958 to 1960 [22-24]. They applied a mean field theory that is able to predict the behaviour of the order parameter at the mentioned phase transition assuming shape anisotropic particles with van der Waals interactions. The derivation shall be presented here in an abbreviated manner.

The Maier-Saupe theory is a Landau theory: The interaction energy is a series expansion in terms of the order parameter close to the energy minimum. This means that the linear term vanishes. The theory considers the interaction with the average orientation of the nearest neighbours. Also one can include that the interaction energy scales as $1 / R^{6}, R$ being the molecular distance, as this is the typical scaling for a van der Waals interaction. Hence, for the molecule $i$ the interaction energy with the nearest neighbours $m_{\mathrm{i}}$ would read as

$$
H_{\mathrm{i}}^{i n t}=-\frac{b}{R^{6}}-\frac{a}{R^{6}} s_{\mathrm{i}} \sum_{k=1}^{m_{\mathrm{i}}} s_{\mathrm{k}},
$$

with $s_{\mathrm{i}}=\frac{1}{2}\left(3 \cos ^{2} \theta_{\mathrm{i}}-1\right)$ depending on the tilt angle $\theta_{\mathrm{i}}$ with respect to the preferred direction, and positive constants $a$ and $b$. In the approximation of a mean field we now simplify

$$
\sum_{k=1}^{m_{\mathrm{i}}} s_{\mathrm{k}} \approx\left\langle\sum_{k=1}^{m_{\mathrm{i}}} s_{\mathrm{k}}\right\rangle=m\langle s\rangle .
$$

Here, $\langle s\rangle$ is the average orientation around the molecule $i$, which is the order parameter as defined in equation (2.1). Introducing the molecular volume $V$ as a proportional of the distance $R^{3}$, the mean field energy of one molecule is given as

$$
H_{\mathrm{i}}^{M F}=-\frac{B}{V^{2}}-\frac{A}{V^{2}}\langle s\rangle s_{\mathrm{i}}
$$

with modified positive constants $A$ and $B$. We find the internal energy per molecule $u$ according to the definition as the expectation value of the possible energies from equation (2.8):

$$
u:=\left\langle H_{\mathrm{i}}^{M F}\right\rangle=-\frac{B}{V^{2}}-\frac{A}{V^{2}}\langle s\rangle^{2}
$$


Keeping in mind that one must not count double the interactions between the $N$ molecules, the total internal energy $U$ is:

$$
U=\frac{N}{2} u=-N \frac{B}{2 V^{2}}-N \frac{A}{2 V^{2}}\langle s\rangle^{2} .
$$

In order to find the free energy of one molecule, it is necessary to calculate the partition function. This is done according to the usual definition of statistical physics as

$$
z(i)=\int e^{-\beta H_{\mathrm{i}}^{\mathrm{MF}}} d \Omega_{\mathrm{i}}, \quad \text { with } \beta=\frac{1}{k_{\mathrm{B}} T} .
$$

The phase space for the molecule used here is given as all molecular orientations in space, which means the integral over the solid angle $\Omega$. Because of the rotational symmetry, the integration over the polar angle gives a factor of $2 \pi$ and the equation reduces to an integral over the azimuthal angle $\theta$ :

$$
z(T, s)=2 \pi e^{\beta \frac{B}{V^{2}}} \int_{0}^{\pi} \sin \theta \exp \left[\frac{1}{2} \beta \frac{A}{V^{2}}\langle s\rangle\left(3 \cos ^{2} \theta-1\right)\right] d \theta
$$

Again the definition of the order parameter for molecule $i$ as $s_{\mathrm{i}}=\frac{1}{2}\left(3 \cos ^{2} \theta-1\right)$ was used. Now the free energy for one molecule is

$$
f=-k_{\mathrm{B}} T \ln [z(T, s)] .
$$

Using that the entropy $s_{\mathrm{E}}$ is

$$
s_{\mathrm{E}}=\frac{u}{T}-\frac{f}{T} \quad \text { and } \quad S_{\mathrm{E}}=N s_{\mathrm{E}},
$$

one finds the free energy of the system as

$$
\begin{gathered}
F=U-T S_{\mathrm{E}} \\
F=N\left[\frac{B}{2 V^{2}}+\frac{A}{2 V^{2}}\langle s\rangle^{2}-k_{\mathrm{B}} T \ln [z(T,\langle s\rangle)]\right] .
\end{gathered}
$$

Finally we find the minimum of the free energy with

$$
\frac{\partial F}{\partial\langle s\rangle}=0
$$




$$
2 \frac{A}{T V^{2}}\langle s\rangle-k_{\mathrm{B}} \frac{\partial \ln [z(T,\langle s\rangle)]}{\partial\langle s\rangle}=0 .
$$

This equation gives the dependence of the order parameter $\langle s\rangle$ only on material constants and the temperature $T$ as an implicit function which, however, cannot be solved analytically.

It is now also possible to calculate the order parameter at the phase transition temperature $T_{\mathrm{p}}$. The condition for the coexistence of the phases is the equality of the chemical potential $\mu$ of the nematic and the isotropic phase. With the definition of the chemical potential

$$
\mu=\frac{\partial F}{\partial N}
$$

and equation 2.16 obtained for the free energy, the chemical potential is

$$
\mu=\frac{B}{2 V^{2}}+\frac{A}{2 V^{2}}\langle s\rangle^{2}-k_{\mathrm{B}} T \ln [z(T,\langle s\rangle)]
$$

Since the order parameter is zero in the isotropic phase, the equilibrium condition becomes

$$
\begin{gathered}
\mu_{\text {iso }}\left(T=T_{\mathrm{p}}\right)=\mu_{\text {nem }}\left(T=T_{\mathrm{p}}\right) \\
\frac{B}{2 V^{2}}-k_{\mathrm{B}} T_{\mathrm{p}} \ln \left[z\left(T_{\mathrm{p}}, 0\right)\right]=\frac{B}{2 V^{2}}+\frac{A}{2 V^{2}}\langle s\rangle^{2}-k_{\mathrm{B}} T_{\mathrm{p}} \ln \left[z\left(T_{\mathrm{p}},\left\langle s\left(T_{\mathrm{p}}\right)\right\rangle\right)\right]
\end{gathered}
$$

For $\langle s\rangle=0$, the partition function of equation 2.12 can be calculated as $z(T, 0)=4 \pi \exp \left(\beta \frac{B}{V}\right)$, so that we finally obtain

$$
\ln [z(T,\langle s\rangle)]+\frac{A}{2 k_{\mathrm{B}} T V^{2}}\langle s\rangle^{2}=\ln (4 \pi)+\frac{B}{k_{\mathrm{B}} T V} .
$$

Again, the result is given in form of an implicit function. Numerical calculations show that the obtained values are close to the experimental values, typically around 0.40 [24].

Although no analytical solution can be given for the temperature dependence of $S$, as mentioned, an approximate solution of the equation is given by

$$
S \approx\left(1-0.98 \frac{T}{T_{\mathrm{NI}}}\right)^{0.22}
$$

with the phase transition temperature from isotropic to nematic $T_{\mathrm{NI}}$ [18]. This temperature dependence is shown in figure 2.7 .

It might be considered to be surprising that the consideration of only the van der Waals interaction leads to a good description of the nematic behaviour without taking into account 


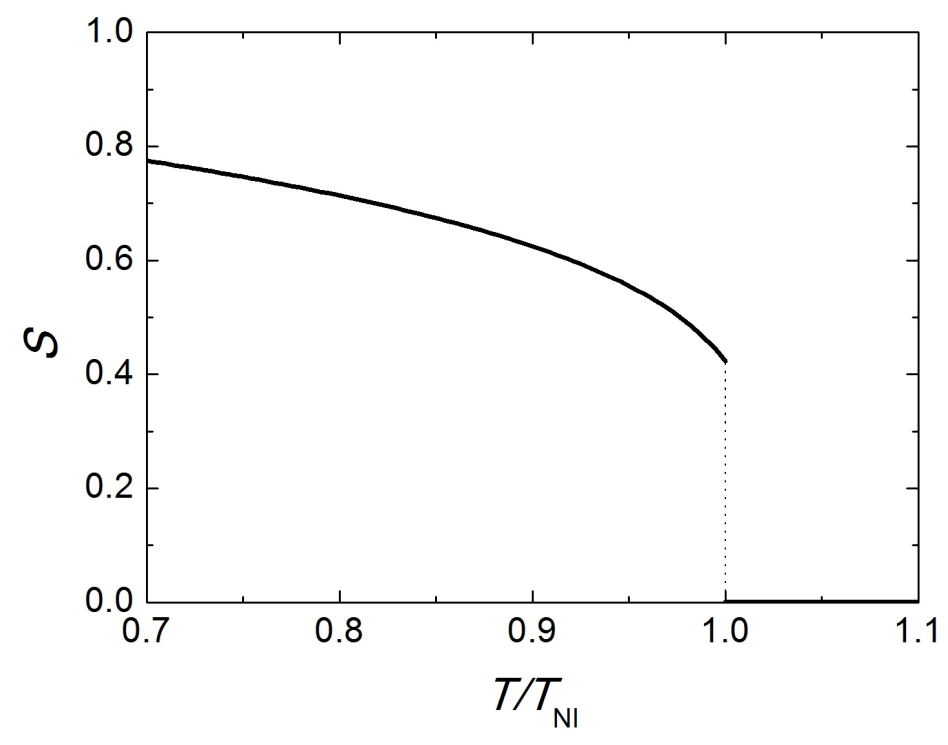

Figure 2.7: Approximate temperature dependence of the order parameter in the nematic phase according to equation (2.24).

other forces that are larger. Particularly forces that are dominating in magnitude like shortrange repulsive forces between the molecules would be expected to play a bigger role. However, it was shown that those forces are similar for the isotropic and the nematic phase, so that the weaker long range forces play the dominant role in the phase transition [25]. This explains the big success of the Maier-Saupe theory.

\subsubsection{Ginzburg-Landau theory}

In this section the transition from the nematic to the smectic A phase and transitions between different smectic phases shall be addresses. As explained in section 2.2, a smectic liquid crystal exhibits a higher probability of finding the center of mass of a molecule in certain regions, generally described as organisation in layers. This can be expressed as a sinusoidal fluctuation of the density around its average $\rho_{0}$. Close to the phase transition one also has to allow for the spacial fluctuation of the density [15], so that we get

$$
\rho(\vec{r}) \approx \rho_{0}(\vec{r})+\rho_{1}(\vec{r}) \cos \left(2 \pi \frac{d}{z}\right)
$$


where $\rho_{1}$ gives the strength of the density fluctuations and $d$ denotes the layer spacing. Here we assumed that the director is oriented in $z$-direction. Considering that the layers might be displaced by $u(\vec{r})$, we define the phase of the layers as

$$
\Phi(\vec{r})=-2 \pi \frac{u(\vec{r})}{d} .
$$

Now, for convenience in calculation, we define the order parameter as a complex number

$$
\psi(\vec{r})=\rho_{1}(\vec{r}) e^{\mathrm{i} \Phi(\vec{r})}
$$

This definition does make sense for smectic ordering as it increases with increasing fluctuations of the density in z-direction (i. e. increasing layering). It also accounts for the proper distance of the layers, as in case of layers that are out of phase $(|u(\vec{r})|>0)$, the real part of $\psi(\vec{r})$ is decreased.

Now the smectic free energy density $f_{\mathrm{SmA}}$ can be written as a Landau expansion which now contain contributions of the powers of $\psi$ but also of its spatial variations [21]:

$$
f_{\mathrm{SmA}}=\alpha|\psi|^{2}+\frac{\beta}{2}|\psi|^{4}+C_{\|}\left|\frac{\partial \psi}{\partial z}\right|+C_{\perp}\left|\nabla_{\perp} \psi\right|^{2} .
$$

The two last terms are the contributions of changes of $\psi$ in space. They account for the different rigidities $C_{\|}$and $C_{\perp}$ within the layers and perpendicular to them respectively (the nabla operator $\nabla_{\perp}$ stands for $\left.\left(\frac{\partial}{\partial x}, \frac{\partial}{\partial y}, 0\right)\right)$. Also close to the phase transition one has to consider all possible deformations of the director described in equation 2.3 , , so that the full total energy density $f_{\text {tot }}$ reads

$$
f_{\text {tot }}=f_{\text {SmA }}+\frac{1}{2}\left[K_{\text {bend }}(\vec{n} \times \operatorname{curl} \vec{n})^{2}+K_{\text {twist }}(\vec{n} \cdot \operatorname{curl} \vec{n})^{2}+K_{\text {splay }}(\operatorname{div} \vec{n})^{2}\right] .
$$

De Gennes showed that in the case of small spatial fluctuations these equations are fully analogous to those of supraconductivity [26]. Consequently, the Ginzburg-Landau theory that describes supraconductivity can fully be applied. For example, a supraconductor does not allow for an internal magnetic field $\vec{B}$, which means that the curl of the vector potential $\vec{A}$ is zero. Similarly, in case of a smectic LC there is no twist deformation, which means that the curl of the director $\vec{n}$ is zero. Additionally, it means that according to this approximation the nematic-smectic A-transition is second order. However, the smectic free energy density 
can also contain cubic terms proportional to $|\psi|^{3}$ which would make the transition a first order transition [15]. In reality both cases are found.

In order to describe the phase transition from smectic $\mathrm{A}$ to smectic $\mathrm{C}$, the magnitude of the tilt $\omega$ relative to the layer normal and the azimuthal tilt angle $\phi$ are used. The order parameter is then

$$
\psi=\omega e^{\mathrm{i} \phi},
$$

which is formally the same as in equation 2.27) for the order in the smectic A phase. Hence, the terms of the free energy density for the smectic $\mathrm{C}$ order also look formally identical to the ones for smectic A (equation (2.28)). However, no terms for bend and twist deformation enter into the total energy, in contrast to equation (2.29). Furthermore, for the transition of the smectic $\mathrm{A}$ to the smectic $\mathrm{C}$ phase, an analogy to the transition to superfluid helium can be drawn [27], for which reason the transition is mainly found to be second order. But again, in the case of larger fluctuations around the phase transition, it can be first order [15]. Both kinds of materials were used for the work presented here.

As mentioned in section 2.2, the hexatic phases exhibit an in-plane order that is characterised by a sixfold symmetry. Hence, for the density changes around a rotational center one can write depending on the polar angle $\phi$

$$
\rho_{\text {hex }} \approx \rho_{0}+\rho_{1, \text { hex }} \cos (6 \phi) .
$$

This equation structurally is of the same shape as the density fluctuations for the smectic A phase in equation 2.25). So it suggests itself to use the same approach again and write the order parameter as

$$
\psi_{\mathrm{hex}}=\rho_{1, \mathrm{hex}} e^{6 \mathrm{i} \phi}
$$

With this method it is again possible to describe the transition to the hexatic phase in a very similar way as the transition from the nematic to the smectic A phase. However, this theory is not adequately describing the real phase transition as for example it predicts erroneous values for the exponent of the specific heat [15]. So far, a consistent theory for the description of the transition to the hexatic phase is lacking. 


\subsection{Thin smectic films}

In this section we want to elucidate the properties of thin smectic films as they are found to show unusual phenomena in regard to the dynamics and the structure formation. The reason for this owes to the combination of thin film effects and anisotropic interaction between the LC molecules, together with the inherent layering of smectics. There are two fundamentally different cases which will be treated separately in the following: First of all one can have stable films on solid substrates (liquid interfaces are not considered here), and secondly one can have free-standing films that do not have a support but are just freely suspended in air.

\subsubsection{Smectic films on substrates}

We consider a smectic liquid film with one interface to a solid substrate and the other one to air. The air interface invariably imposes a homeotropic anchoring (i.e. perpendicular to the surface) on the liquid crystals with an anchoring strength that typically is larger than any kind of anchoring strength achievable for solid substrates [5]. Therefore, the perpendicular orientation at the free surface is a fixed parameter for all the considered films. For the solid interface one can prepare either planar or homeotropic anchoring. However, for the case of homeotropic anchoring one gets a homogeneous director orientation throughout the whole film that is less interesting.

More appealing is the case where the substrate favours planar anchoring as this induces conflicting boundary conditions. To satisfy these, there are various possibilities to fulfill both anchoring conditions by bending the structure within a film possessing a thickness in the order of one micron. Depending on the specific properties of the solid material, different structures can be observed. Of special interest have been the so called focal conic domains (shown in figure 2.8) that are formed in case of a random planar anchoring. They have been discovered already more than a hundred years ago [28] but are still under investigation as they were shown rather recently to have potential application for the formation of photonic crystals [9] or for the creation of superhydrophobic surfaces [10]. Depending on the elastic constant $K$ (see section 2.3) and the surface tensions, one finds the threshold for the formation of focal conic domains to be at a thickness of some hundred nanometers [29].

Another possible arrangement of thin films of a smectic LC is obtained in the case of uniform planar anchoring. The minimisation of the free energy leads to the formation of hemicylinders [31, 32]. The difference is that for focal conic domains defect lines suffice to 

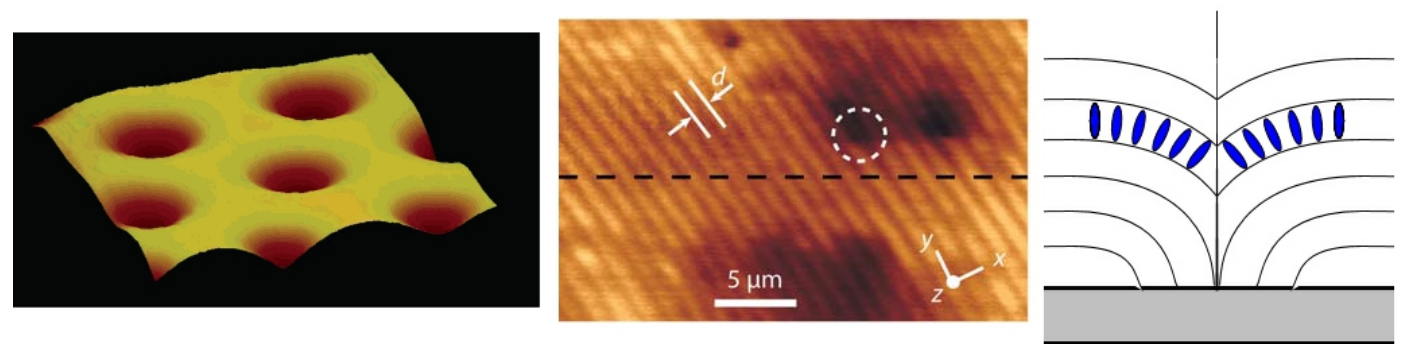

Figure 2.8: AFM images of focal conic domains (left, image taken from [30]) and hemicylinders (middle, image taken from [31]). The sketch for the cross section (right) is the same for both structures and shows the reason for the surface depression in order to fulfill both boundary conditions. However, only for the random planar surface anchoring the formation of circular structures is possible.

fulfill both boundary conditions. However, for planar anchoring the circular director structures are not possible, resulting in the formation of the defect walls of hemicylinders. The structure is shown in figure 2.8 .

A particularity of smectic materials is that they can also be prepared as molecularly thin films. This is in contrast with the behaviour of the nematic phase due to their tendency to accommodate the boundary condition by separation in two well defined values of film thickness, where one is only the prewetting film and the second is thick enough that bend deformation can reorient the molecules by $90^{\circ}$ [33]. This does not occur in smectic films as bend deformation is forbidden in this case.

However, in the case of smectic phase the stability of thin films of certain combinations of liquid crystal and substrate can sometimes be controversial. An example that is important for our experiments is the standard mesogen $8 \mathrm{CB}$ (see chapter 4.1) on a silicon substrate. The apparently same system sometimes shows dewetting [34, 35] and sometimes complete wetting [36, 37]. As we will see later, we agree with the latter observation. However, the observation of the possibility to have stable films with only one layer thickness and three layers thickness is beyond dispute. This conformation of the material is found to be very stable as it is consistently observed on water [38], for spreading droplets [36], and for evaporated films [34]. The corresponding molecular structure is shown in figure 2.9. In chapter 6 we will show how to use this stable configuration in order to build up homogeneous films with an arbitrary number of layers and subsequently use those well-defined films to manipulate the surface structure in a desired way. 


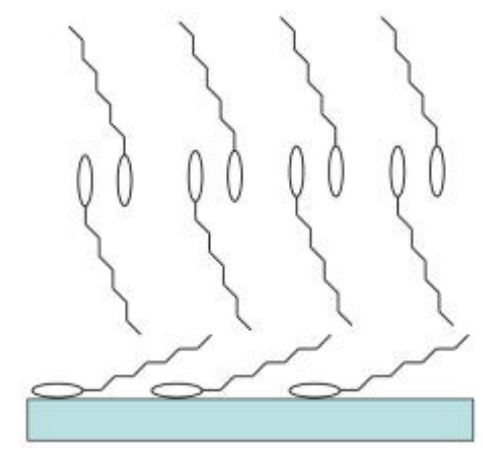

Figure 2.9: Sketch of the molecular arrangement for an 8CB film with a thickness of 3 molecular layers on a silicon substrate.

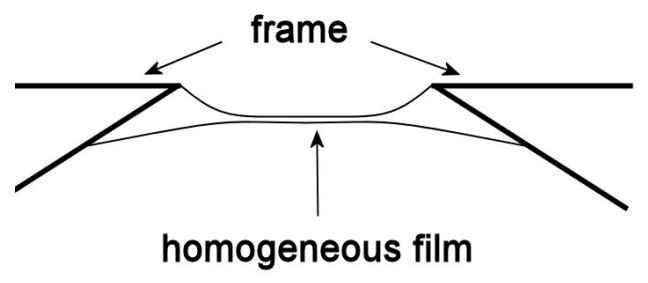

Figure 2.10: Sketch of a free-standing film spanned over a supporting frame. Apart from meniscus of the boundary region, one obtains a very homogeneous film in the middle.

\subsubsection{Free-standing smectic films}

As mentioned above, it is possible to have a film that is freely suspended over a supporting frame and only has two air interfaces. The formation of such films is stable for all smectic phases. A schematic image is shown in figure 2.10. It is found that with this method it is possible to create films that are homogeneous at a molecular level. This means that there is a well defined number of molecular layers over macroscopic length scales. The number of layers that can be stably prepared ranges between two and hundreds of layers. The interest in such films particularly comes from the fact that they can serve as two-dimensional model systems wherein the continuous transition from two to three dimensions can be studied.

The general structure of free-standing films is very similar to the one of soap bubbles but offer the advantage of higher variability of the film thickness and better stability as there is no solvent that gets drained out. 
The preparation of a free standing film is very straightforward. By simply drawing smectic material over a supporting frame of arbitrary shape using a sharp edge, one obtains a heterogeneous free-standing film that equilibrates to a homogeneous film thickness in a time of minutes or hours, depending on the material in use. If there are regions with a higher or lower film thickness, they get stabilised if their radius exceed a critical radius [39]. The film conclusively can get thicker or thinner by taking up material or giving material to the meniscus. Usually the thinnest region shows to be dominant and grows till it covers the whole film upto the meniscus [40].

In order to keep the film stable, there has to be a tension exerted from the meniscus on the film to counter the surface tension $\gamma$ [39]. Additionally in elastic films as the smectic ones, the films can support a pressure difference $\Delta p$ with respect to the surrounding air that encloses the film [41], contrary to classical fluids that are always in pressure equilibrium to the surrounding gas. The tension $\tau$ along the smectic membrane is then

$$
\tau=2 \gamma+\Delta p h
$$

with the film thickness $h$. As this tension has to result from the meniscus we see that the meniscus plays a crucial role both in the stabilization of the whole film and serving as a material reservoir. Also the shape of the meniscus can be used to measure the tension $\gamma$ of the film with the help of the macroscopic angle at which the meniscus meets the flat film [42].

A manipulation of the film thickness is usually very difficult as one would have to nucleate a sufficiently large region of different thickness. Only for the removal of a small number of layers, a method locally heating up the sample can be employed [41]. In a simplified image of this process, one can imagine that the film gets softened around the heated area, making the film more vulnerable to the external air pressure so that a surface layer can be pushed to the outside regions [43].

Also for isotropic liquids, ordering effects in ultrathin films in the form of layering are observed (see e.g. [44-46]). As liquid crystals already show an inherent tendency to arrange in layers as in the smectic phase, this surface ordering gets even more pronounced. Generally not only solid interfaces but also the free surface seems to favour the more ordered LC phase. The interface breaks the translational symmetry of the material and induces a positional (smectic) order at the surface. This smectic ordering at the surface can be observed for nematic-smectic A [47] or isotropic-smectic A transitions [48]. Consequently, the local order parameter close to surfaces is also different from the one in the bulk [40]. 


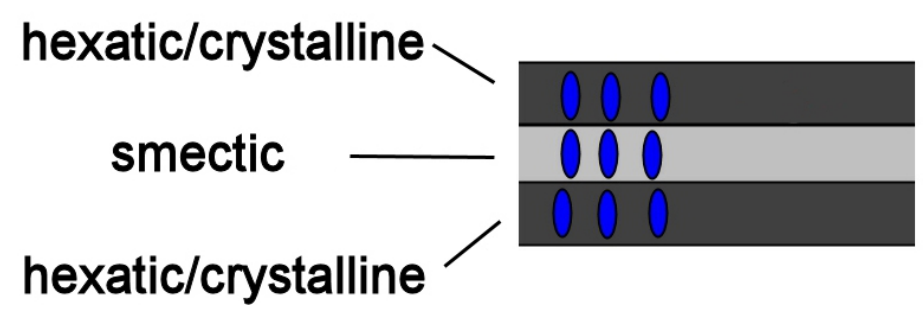

Figure 2.11: Schematic sideview of an axially inhomogeneous film with frozen outside layers (in hexatic or crystalline smectic state) and less ordered phase (smectic) in the middle.

Apart from the more obvious case of the smectic ordering due to the interface, other transitions to higher ordered states can be triggered by the free interfaces. For the transition from smectic A to smectic C, usually a continuous tilt is induced at the surface [49]. The shift of the phase transition temperature for the surface layer can be as high as tens of Kelvins [50].

Additionally, in-plane ordering can be induced by the free surface [51]. An overview over those surface-mediated transitions to the hexatic phases and the related processes are given in [52]. When approaching the transition temperature to hexatic or crystalline smectic phases, usually the outer layers at the air interface freeze at significantly higher temperatures [53, 54], which leads to a thin slice of less ordered LC material between higher ordered layers as shown in figure 2.11. These films are interesting because they provide laterally inhomogeneous films with a phase boundary that is huge compared to the volume. The molecular behaviour in such a situation will be analysed in this work.

Another effect in free-standing films close to their phase transitions is that they can sometimes be heated into the bulk nematic or bulk isotropic temperature range. The film does not rupture suddenly but gets thinner layer-by-layer as shown in figure 2.12. This behaviour was first observed for fluorinated compounds [55] but later also reported for standard LCs like $8 \mathrm{CB}$ [42]. When starting with a free-standing film of thickness $l_{0}$, the film starts to get thinner when heating over the bulk transition temperature of the smectic phase $T_{0}$. The thickness $l(T)$ then scales with the temperature according to the power-law

$$
l(T)=l_{0}\left(\frac{T-T_{0}}{T_{0}}\right)^{-v} .
$$

As can be seen by the power law dependence, the thinning process starts at the phase transition temperature. 

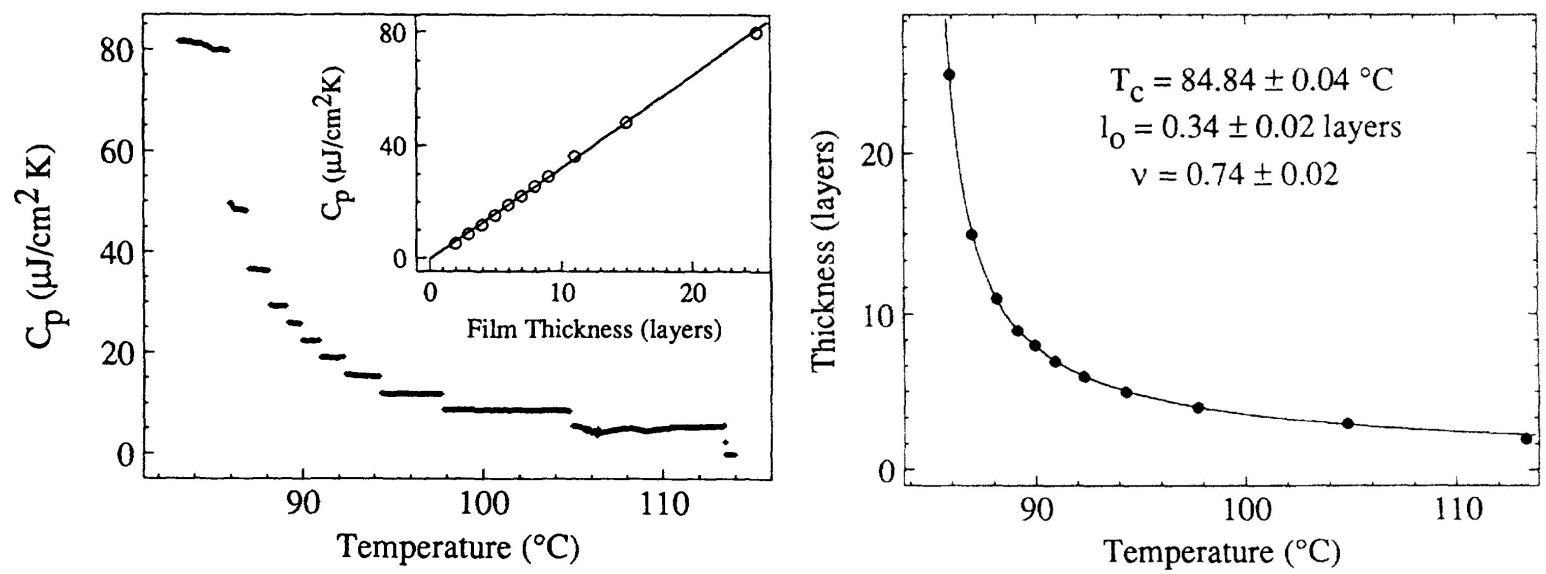

Figure 2.12: Layer-by-layer thinning of overheated free-standing films. Left: Stepwise change of heat capacity of the film shows removal of individual layers. Right: Thickness-dependence obeys the power law of equation (2.34). Diagrams taken from [55]. 


\section{Chapter 3}

\section{Diffusion analysis using single molecule tracking}

In this chapter, the method that was used to measure diffusion in thin films is presented. The first section concerns the fundamental equations that govern diffusion in general. This is followed by a short section on how the anisotropic structure influences the diffusive behaviour in the case of liquid crystals and which methods can be used to measure diffusion at molecular level. The chapter concludes with a presentation of the method that was employed for this thesis using dye molecules to probe the diffusion within the host matrix.

\subsection{Fundamentals of diffusion}

The classical definition of diffusion is done via an inhomogeneous liquid, for example within a container one half of which is filled with material dissolved in a solvent and the other half contains only the pure solvent. As the two regions get in contact, a material transport is observed such that the dissolved material spreads out into the regions with pure solvent until an equal distribution of material is obtained everywhere. As it was found experimentally, the net flux of the material $j$ is proportional to the concentration gradient in the solvent. The proportionality factor is called as the diffusion coefficient $D$ and one gets Fick's first law (in one dimension):

$$
j=-D \frac{\partial c}{\partial x}
$$

with the gradient of the concentration $\partial c / \partial x$ along the $x$-axis. It was shown for the first time theoretically by Einstein that the macroscopic appearance of the directed motion can be 
explained by the thermal motion (Brownian motion) of the molecules [56]. Simply the fact that there are more molecules moving away from the higher concentrated regions than in the opposite direction gives the impression of a material flux. The diffusion coefficient can thus be related to the microscopic motion of the molecules. For a small distance $\Delta x$ traveled in time $\tau$ one finds the relation

$$
\Delta x^{2}=2 D \tau
$$

This equation is called Einstein-Smoluchowski equation. In a microscopic image, one can also assume that the path travelled by a molecule is discretised by the mean free path $\lambda$ of the molecules. Especially for a gas one can now substitute the mean particle velocity $\bar{v}$ for $\frac{\lambda}{\tau}$ and directly relate the diffusion coefficient to microscopic quantities [57]:

$$
D=\frac{1}{2} \lambda \bar{v}
$$

We see that in this approach the diffusion coefficient has no relation to any gradient of the concentration anymore. Thus, the importance of equation (3.2) can be seen in the bridge that it builds from the motion at molecular level to the diffusion that was originally a macroscopic property.

As the movements in all spatial directions are independent of each other, one can also write equation 3.2 for $\Delta y$ and $\Delta z$, and find the squared displacement as $\Delta r^{2}=\Delta x^{2}+\Delta y^{2}+\Delta z^{2}$. Generally we find for the diffusion in $n$ dimensions the equation

$$
\Delta r^{2}=2 n D \tau
$$

One can also consider the probability density $P$ to find a particle - that originally was located at the point $\overrightarrow{r_{i}}-$ at the point $\overrightarrow{r_{j}}$ after a time interval $\Delta t$. From the theory of random walks it is known that after a time that is long compared to the time between two collisions, one finds the Gaussian distribution [58]

$$
P\left(\left|\vec{r}_{\mathrm{i}}-\vec{r}_{\mathrm{j}}\right|, \Delta t\right)=\frac{1}{(4 \pi D \Delta t)^{\frac{3}{2}}} \exp \left(-\frac{\left(\overrightarrow{r_{\mathrm{i}}}-\overrightarrow{r_{\mathrm{j}}}\right)^{2}}{4 D \Delta t}\right) .
$$

As we have seen, the diffusion is related to the thermal motion of the molecules and thus gets faster for increasing temperature $T$. On the other hand the diffusion is limited by the 
internal friction $f$ in a liquid. Those properties are expressed by the Stokes-Einstein relation [57]:

$$
D=\frac{k_{\mathrm{B}} T}{f} .
$$

Using the Stokes' law we can now relate the diffusion coefficient to the dynamic viscosity $\eta$ as

$$
D=\frac{k_{\mathrm{B}} T}{6 \pi \eta a}
$$

with $a$ being the diameter of the diffusing particle. This connection between $D$ and $\eta$ gives a chance to measure the viscosity of liquid crystals via the diffusion. This is particularly important as the viscosities are hard to measure in liquid crystals since typical shear experiments tend to destroy the alignment of the LC material.

The temperature dependence of the diffusion coefficient is generally observed to be of the Arrhenius-type, i.e. shows an exponential behaviour. For liquids, however, the explanation cannot be carried out as for solids. In the latter an activation energy needs to be overcome and the probability for this scales exponentially with temperature (Boltzmann distribution). Still it was shown that in a microscopic image, where the molecules have a thermal random motion and occupy a new spot as soon as there is a minimum free volume around them, the temperature dependence has the same shape as for solids [59]. Assuming a finite glass transition temperature of the liquid $T_{\mathrm{g}}$, the equation reads as

$$
D=D_{0} \exp \left(-\frac{A}{T-T_{\mathrm{g}}}\right)
$$

with a constant $D_{0}$ that only weakly depends on temperature. $A$ is a constant that describes the free volume necessary to change the position of a molecule.

\subsection{Diffusion in liquid crystals}

The ordering of the molecules in liquid crystals leads to an anisotropy also in the diffusion coefficient. This statement is generally true for all the possible LC phases [60]. However, once the distinction between the diffusion coefficient parallel to the director $D_{\|}$and the diffusion coefficient perpendicular to it $D_{\perp}$ are introduced, the equations discussed in section 3.1 should hold good. The situation gets slightly more subtle if we consider a film in the smectic A phase as we have a varying potential of the molecules in the direction of the layer normals (parallel 
to the director). This will, however, not influence the diffusion in the layer planes as this is not affected by the potential modulation and we can write as in equation (3.8)

$$
D_{\perp}=D_{0, \perp} \exp \left(-\frac{A}{T-T_{\mathrm{g}}}\right)
$$

Contrary to that, the temperature dependence of the diffusion parallel to the director gets more complicated. However, since the work presented here will only deal with the perpendicular component, this case shall not be considered in detail. Roughly speaking the prefactor of equation (3.8) gets a stronger temperature dependence [61, 62] .

The difference between the diffusion parallel and perpendicular to the director is generally not larger than a factor of two. For a nematic liquid crystal the diffusion parallel to the director always is faster as the hydrodynamic diameter of the molecule from equation (3.7) is smaller when moving in this direction. For smectic LCs the movement in the direction parallel to the director is hindered by the potential walls between the layers. Depending on which effect dominates, the diffusion coefficient $D_{\|}$or $D_{\perp}$ can be larger. Even a crossover of the dominating effect depending on the temperature can be observed [60].

The most popular ways to measure the self-diffusion in liquid crystals are Nuclear Magnetic Resonance (NMR) and Quasielastic Neutron Scattering (QENS) [60]. For QENS a nearly monoenergetic neutron beam is scattered by the LC sample. From the energetic broadening one can deduce the mobility of the molecules in the sample (for example [63]). However, for this method one has the problem that other nuclear motions like oscillations can interfere with the diffusion measurements [64]. Also the small dimensions of typical QENS samples hinder the alignment of the material so that no defined measurement of $D_{\|}$and $D_{\perp}$ is possible.

These problems encountered in QENS are overcome using pulsed field gradient NMR. For this method the permanent magnetic field always present in NMR is overlaid by a pulse of an inhomogeneous magnetic field, for example a sinusoidal one. This pulse provides a maximum magnetisation in the regions of the maximum magnetic field. Thereafter, because of the diffusion, the induced magnetisation pattern will smear out [65], thus giving information about the diffusion within the system as this will happen faster in the case of a faster diffusion. However, this technique is not applicable for the measurement of the diffusion within freestanding films envisaged in this thesis. The reason is that first of all the molecules have to be aligned in the direction of the permanent magnetic field. As free-standing films only allow the orientation perpendicular to the surface, a measurement of the diffusion within the layers is not possible. Secondly, the temperature control is done by an air flow around the sample, which 
induces flow also in a free-standing film. The diffusion would consequently be dominated by the convection and no temperature control would be possible for diffusion measurements. Hence, also NMR cannot be used for our samples.

A more recent approach is to use dye molecules to probe the mobility in the LC matrix. For free-standing films fluorescence recovery after photobleaching was used to sample the diffusion in films when approaching the two-dimensional limit [43]. Lately single molecule methods were also used starting with fluorescence correlation spectroscopy [66]. The most recent approach is single molecule tracking, where only a very limited number of publications exists till now [67-69]. In the following paragraph it will be presented why this technique is useful for the diffusion analysis especially in ultrathin films.

\subsection{Fluorescent probe molecules for diffusion analysis}

The idea of diffusion analysis via single molecule tracking is to dissolve a tiny amount of dye molecules in the surrounding host matrix and then optically follow the movement of the individual molecules. The advantage is that the concentration of guest molecules is smaller than the normal impurity of the material so that no disturbance is induced. Furthermore, the analysis is done optically so that the method is completely non-invasive.

Contrary to all other methods shortly introduced in section 3.2 one directly gets access to the movements at a molecular level as there is no averaging over the whole ensemble of molecules. This is of course most advantageous when probing heterogeneous systems. Such a property is opening up investigations ranging from fundamental problems like diffusion in nanoporous systems [70] to the application of cell dynamics in living cells [71].

In the following paragraphs the fundamentals of fluorescence imaging will be explained before the conditions, accuracy, and limitations of the single molecule methods are finally discussed.

\subsubsection{Fluorescence imaging}

All kinds of fluorescence microscopy is based on the property of dye molecules to absorb light of a certain wavelength and emit light of a different wavelength. The physical processes involved are usually depicted in an energy diagram called as Jablonski diagram, which is shown in figure 3.1. It shows the energy of the highest occupied molecular orbit (HOMO level) and the lowest unoccupied molecular orbit (LUMO level) of one molecule. We consider 


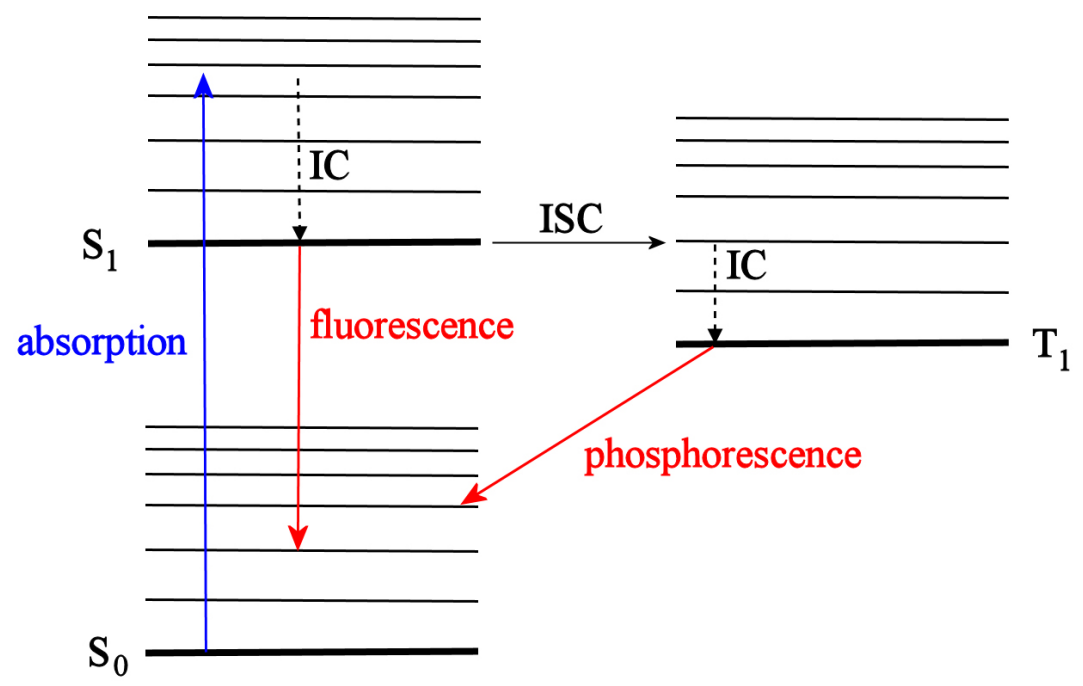

Figure 3.1: Energy diagram of a molecule with the HOMO level $S_{0}$ and the excited electronic states $S_{1}$ and $T_{1}$ with the corresponding vibrational states. Abbreviations: IC - internal conversion, ISC - intersystem crossing (explanations in the text).

a molecule in the ground state (HOMO) which is the singlet state $\mathrm{S}_{0}$. If a photon is absorbed by the molecule in the ground state, the molecule is excited into the singlet state $S_{1}$ or one of the vibrational or rotational modes on top of $S_{1}$. The minimum energy required for the absorption is $E_{\text {abs }}>E\left(\mathrm{~S}_{1}\right)-E\left(\mathrm{~S}_{0}\right)$ as readily visible in the energy diagram. Of course also excitations in higher electronic states are possible but this case shall not be considered here. The first step in de-excitation of the molecule is governed by the characteristic times that the different processes take. The fastest is the vibrational relaxation with time scales of $10^{-12} \mathrm{~s}$ up to $10^{-10} \mathrm{~s}$ [72] during which the molecule relaxes back into the lowest vibrational level of $\mathrm{S}_{1}$; a process which is called internal conversion. As we consider the molecules in a surrounding matrix it can always "lose" energy in the collision with other molecules.

The process that is observed as fluorescence light is then the relaxation from $\mathrm{S}_{1}$ onto one of the vibrational levels above $S_{0}$ on time scales of $10^{-10}$ to $10^{-7} \mathrm{~s}$ [72]. The energy of the emitted photon is $E_{\mathrm{em}}<E\left(\mathrm{~S}_{1}\right)-E\left(\mathrm{~S}_{0}\right)$. One directly sees that the energy of the emitted photon is smaller than the energy of the absorbed photon. This red shift is the reason why it is always possible to spectroscopically separate the fluorescent light of a sample from its reflected light. This property is the key to all fluorescence imaging. 
The competing luminescent process is phosphorescence. For this, the spin of the excited electron has to be flipped when the electron is in the $S_{1}$ state (called intersystem crossing). After the relaxation into the ground state of $T_{1}$ the molecule can relax back to $S_{0}$ by emission of a photon. Generally the transition from a triplet state to a singlet state or vice versa is forbidden in quantum mechanics. However due to spin-orbit coupling one gets a finite probability for this process [72]. The low probability gives rise to higher stability of the state $T_{1}$ which results in time scales of relaxation in the range of milliseconds to seconds [73].

The most important property of fluorescence - the red shift of the emitted light - was already mentioned. Furthermore, usually one observes the emission and the absorption spectra to be symmetric. The reason is that the rotational and vibrational levels above $S_{0}$ and $S_{1}$ are very similar. According to the Franck-Condon principle one obtains similar transition probabilities from the ground state $S_{0}$ into a vibrational state of $S_{1}$ and vice versa. Also from the considerations of the molecular processes it becomes obvious that the emission spectrum does not depend on the excitation wavelength as the fluorescence always starts from the lowest level of $S_{1}$.

From the above simplified description, one would expect a discrete spectrum as all the energetic states are separated. However, the emission wavelength of a considered molecule also depends weakly on the immediate surrounding of the molecules. As this is different for every emitting molecule, the observed emission wavelengths are smeared out and one observes a continuous spectrum.

Of course there is a number of processes that can lead to a non-radiative transition to the ground state. Examples for this are collisional quenching where molecules transfer the energy to neighbouring molecules upon contact, resonant energy transfer in which the emission spectrum is overlapping the absorption spectrum of a nearby molecule, and photochemistry [73]. All these effects decrease the number of photons emitted per photon absorbed (called the quantum yield).

\subsubsection{Single molecule tracking}

The crucial condition for tracing single dye molecules is that the surrounding material shows practically no fluorescence by itself. If this is fulfilled and the signal-to-noise ratio ( $S N R)$ is high enough, the recording of the trajectories of the molecules becomes possible. These conditions were matched in 1990 by Brooks Shera et al. [74] when for the first time the motion of single fluorescent molecules was tracked. 
Conceptually the method of single molecule tracking is similar to single particle tracking were mesoscopic objects probe a sample. Both methods take movies of the movement of objects and deduce the mobility of the probe in the sample. However, dye molecules - having a dimension of around one nanometer - should also be able to probe smaller regions in the size of nanometers. On the one hand optical microscopy has to obey to the classical Abbe limit of two points not being distinguishable if they are closer than a distance $d$ of

$$
d=\frac{\lambda}{2 N A},
$$

with the emission wavelength $\lambda$ and the numerical aperture $N A$. On the other hand the knowledge of the point spread function (PSF) of the imaged object allows one to localise an emission center a lot more precisely. As the dye molecules are very small compared to the final PSF, they can be considered as point-like emitters. Then the PSF is radialsymmetric and only depends on the deflection angle $\theta$. In total one obtains an Airy function [75] where the intensity distribution has the form

$$
I(\theta)=4 I_{0}\left(\frac{J_{1}(k a \sin \theta)}{k a \sin \theta}\right)^{2},
$$

with the maximum intensity $I_{0}$, the wave vector $k$, and the radius of the aperture $a$. The function $J_{1}$ is the Bessel function of first kind and first order. The Airy function is shown in figure 3.2. Calculating the center of the PSF gives the chance to have a precision in the localisation of an object that is a lot higher than the resolution limit of optical microscopy. In experiments an accuracy better than $1.5 \mathrm{~nm}$ was obtained even for slowly moving objects [76]. However, for this precision long illumination times of the camera are required in order to get a strong enough signal (in the mentioned paper for example $0.5 \mathrm{~s}$ ) which is not applicable for diffusion processes in liquid crystals as frame rates of around $50 \mathrm{~Hz}$ are necessary.

In order to find the the real localisation accuracy of an experiment the pixel structure of the detector also plays an important role as this decides how well the detected distribution can be fitted to the PSF. Additionally, the $S N R$ decides how reliably the center of a PSF can be found. Including the detector geometry in a constant factor one finds the accuracy $\Delta x$ to be inversely proportional to the $S N R$ [77]:

$$
\Delta x \propto \frac{1}{S N R} .
$$

The chances to increase the localisation accuracy are therefore limited. Possible ways to increase the signal are for example using a higher excitation intensity, using a dye molecule 


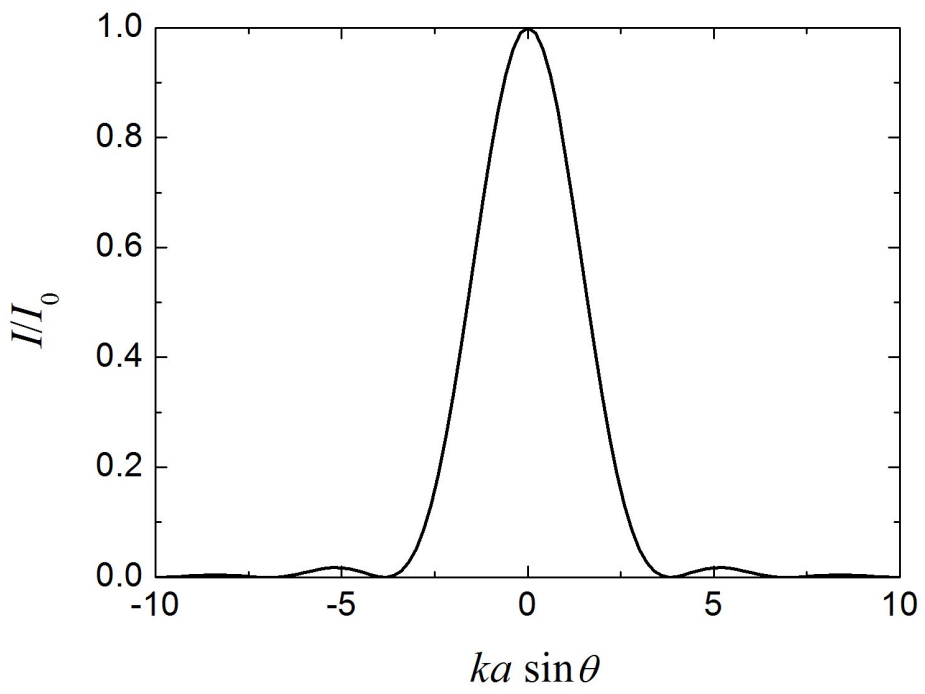

Figure 3.2: Radial distribution of the light intensity depending on the deflexion angle $\theta$ for light of wave vector $k$ if imaged with a lens of aperture $a$ (Airy function).

that is on the one hand suited for the used laser light and on the other hand has a high quantum yield, and working with long exposure times of the camera. The first is restricted by the necessity to keep the sample unchanged and the latter by the movement of the molecules. Another possibility is to keep the noise as low as possible mainly by cooling the camera in order to reduce the thermal noise. Other sources of noise come directly from the sample like reflected light that was not filtered out completely or Raman-scattered light from the sample. This light is red-shifted. It can thus not be separated from the fluorescent light and contributes as an inevitable source of background noise.

After the centers of the molecules are identified in the frames of the movie, the trajectories can be reconstructed as long as the molecules do not come too close to each other. This would lead to an intersection of the trajectories and lead to inaccurate results for the diffusion. In order to avoid the frequent occurrence of this effect, there should only be a small number of molecules in the focus at the same time. Once a trajectory of length $N$ is reconstructed, the diffusion coefficient can be calculated according to equation (3.4). To get a higher statistical reliability of the diffusion coefficient it is desirable to average over as many squared displacements as possible. We get

$$
L=\frac{N(N-1)}{2}
$$




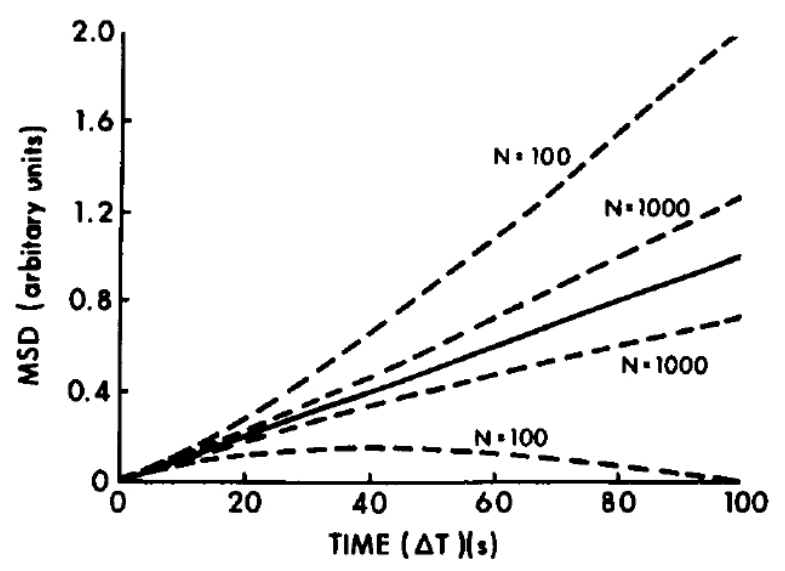

Figure 3.3: Statistical error in calculated mean squared displacements for a trajectory of length $N$. The solid line gives the average, dashed lines give the statistical uncertainty. (image taken from [78])

different pairs of frames over which we can average, which means that the accuracy of the average increases with the square of the number of frames considering $L$ to be a measure for accuracy. After knowing the mean squared displacements for different time lags, one can find the diffusion constant following equation (3.4). However, the average of short time lags is known more precisely as one can average over more pairs of frames. For a time lag of $n$ individual time steps one finds that the variance $\sigma^{2}$ increases approximately proportional to $n$ [78] (see figure 3.3). That is why different time lags $\Delta t$ should be weighted with factors inversely proportional to the variance. It also needs to be considered that different pairs of frames are correlated with each other when averaging over all pairs. However, the advantage of the higher statistical precision when using all possible pairs outbalances the disadvantage of the correlation [79]. When analysing a bigger number of trajectories, their diffusion coefficients can be approximated as a Gaussian distribution [79]. 


\section{Chapter 4}

\section{Methods and experimental techniques}

This chapter starts with the introduction of the chemicals that were used for the experiments. Mainly the different mesogens that were studied will be presented including their different phases and their physical properties. Then the applied experimental techniques will be explained with the focus on the single molecule microscope that was built in the course of this work.

\subsection{Materials}

For the first part of the experiments using free-standing films, the formation of stable films is considered to be a common feature for all smectic mesogens. However, some mesogens show the property that the thickness is not constant over time: The material tends to flow towards the center of the film. As the intention was to study thin films, such materials could not be used. Apart from this, the restriction was mainly that the liquid crystal material had to be clean enough to allow the measurement of individual dye molecules as described in chapter 3.3.2. For this purpose, the purity provided by the supplier is not decisive as the concentration of those impurities is invariably higher than the one of the dye molecules. The possibility to analyse single molecule diffusion is decided by the amount of luminescent molecules already existing in the "pure" LC material. As no mesogens are produced particularly for the intended analysis method it is sometimes a matter of chance if a material can be used. Even the material from the same supplier can lose its usability from one batch to the next. Nevertheless, apart from the inconvenience that not every material can be studied, it is simple to decide if a certain 


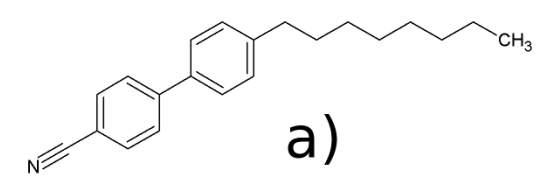<smiles>CCCCCCCOc1ccc(CNc2ccc(CCCC)cc2)cc1</smiles><smiles>CCCCCCCCOc1ccc(-c2ncc(CCCCCCCC)cn2)cc1</smiles><smiles>CCCCCCCCCOc1ccc(CNc2ccc(CCCC)cc2)cc1</smiles><smiles>CCCCCCCOc1ccc(-c2ccc(OC(=O)C(Cl)C(C)CC)cc2)cc1</smiles>

$1 \mathrm{~nm}$

Figure 4.1: Molecular structure of the employed mesogens with their short names used later in the text: a) $8 \mathrm{CB}$, b) pyrimidine, c) 70.4, d) 90.4, e) $\mathrm{C} 7$.

material can be used by looking at the pure material under a single molecule microscope (see section 4.5).

Although restricted by the purity of the available material, the intention was still to look at free-standing films of liquid crystals with different phase behaviour. All of them had a smectic A phase that was analysed for the film thickness dependence of the diffusion. Then the changes in diffusion close to different kinds of phase transitions were studied. The structure of all the molecules that will be mentioned is shown in figure 4.1. The first is 4-n-octyl-4'-cyanobiphenyl (8CB, Synthon Chemicals) that was studied at the phase transition crystalline-smectic A and smectic A-nematic. The advantage of this material is that it is a commonly used LC and the properties are well-known. The phase structure of $8 \mathrm{CB}$ is the so called smectic $\mathrm{A}_{\mathrm{d}}$ phase which means that the smectic layers consist of double layers of molecules with opposite orientation of neighbouring molecules with the head groups being side-by-side so that the smectic layer spacing is around 1.4 times the molecular length [80]. The reason for this arrangement is the strong electric dipole moment of the molecules of around $5 \mathrm{D}$ [81]. This comparably strong dipole moment (for example more than double the dipole moment of water) 
orients the nearest neighbours in the opposite direction so that the resulting dipole moment of one smectic layer in bulk is zero.

The second mesogen is 5-n-octyl-2-[4-(n-octyloxy)-phenyl]-pyrimidine (will be called pyrimidine). This is a material which has a low dipole moment and forms smectic layers in the order of the molecular length. The pyrimidine shows a smectic A and a smectic $\mathrm{C}$ phase. There are no existing studies about the pure material and it is mainly used in mixtures with other molecules [82].

Thirdly, two molecules from the so called nO.m series possessing a transition to a phase with an in-plane ordering were also studied. The first one is N-(4-n-heptyloxybenzylidene)4'-n-butylaniline (7O.4) and has a transition to the smectic G phase. The second one is N(4-n-nonyloxybenzylidene)-4'-n-butylanine (90.4) which was studied mainly at the transition from the smectic $\mathrm{C}$ to smectic $\mathrm{F}$ phase.

Finally 4-(3-methyl-2-chloropentanoyloxy)-4'-heptyloxybiphenyl (C7) was used as it shows a first order phase transition from smectic A to smectic $\mathrm{C}$ [83]. Because of the chirality of the molecule it actually forms a smectic $\mathrm{C}^{*}$ phase where the tilt direction of the molecules is rotated from one layer to the next. The phases of all the used mesogens together with the phase transition temperatures are shown in Table 4.1 .

\begin{tabular}{|c|c|}
\hline mesogen & phase sequence \\
\hline \hline $8 \mathrm{CB}$ & cryst $-22^{\circ} \mathrm{C}-\mathrm{sm} \mathrm{A}-33.8^{\circ} \mathrm{C}-$ nem $-40.8^{\circ} \mathrm{C}-$ iso \\
\hline pyrimidine & cryst $-28.7^{\circ} \mathrm{C}-\mathrm{sm} \mathrm{C}-57.0^{\circ} \mathrm{C}-\mathrm{sm} \mathrm{A}-64.5^{\circ} \mathrm{C}-$ nem $-70.3^{\circ} \mathrm{C}-$ iso \\
\hline $7 \mathrm{O} .4$ & cryst $-32.6^{\circ} \mathrm{C}-\mathrm{sm} \mathrm{G}-59.9^{\circ} \mathrm{C}-\mathrm{sm} \mathrm{C}-64^{\circ} \mathrm{C}-\mathrm{sm} \mathrm{A}-71.4^{\circ} \mathrm{C}-$ nem $-73.9^{\circ} \mathrm{C}-$ iso \\
\hline 90.4 & cryst $-50^{\circ} \mathrm{C}-\mathrm{sm} \mathrm{G}-67^{\circ} \mathrm{C}-\mathrm{sm} \mathrm{F}-69.5^{\circ} \mathrm{C}-\mathrm{sm} \mathrm{A}-82^{\circ} \mathrm{C}-$ iso \\
\hline $\mathrm{C} 7$ & cryst $-?-\mathrm{sm} \mathrm{G}-45^{\circ} \mathrm{C}-\mathrm{sm} \mathrm{C}-55^{\circ} \mathrm{C}-\mathrm{sm} \mathrm{A}-62^{\circ} \mathrm{C}-$ iso \\
\hline
\end{tabular}

Table 4.1: Phase transition temperatures of the used mesogens 8CB [84], pyrimidine (measured by texture changes using polarisation microscopy), 70.4 [85], 90.4 [86], and C7 [87].

For the measurements of films on substrates, the only liquid crystal in use was the already mentioned $8 \mathrm{CB}$. The reason why no other material was studied is simply that no other mesogen was found that would form films of arbitrary film thickness. The same preparation precedure as for $8 \mathrm{CB}$ was tried for the compounds $9 \mathrm{CB}$ and $10 \mathrm{CB}$ - which have the same chemical structure as $8 \mathrm{CB}$ just with a longer alkyl chain - and pyrimidine. However, all of 


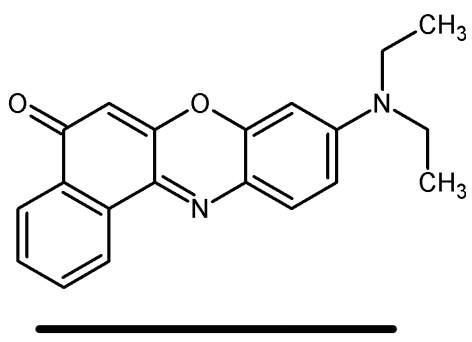

$1 \mathrm{~nm}$

Figure 4.2: Molecular structure of Nile red

them showed dewetting although the two other cyanobiphenyls would be expected to show the same behaviour as they have very similar interaction properties with the substrate.

For the diffusion measurements the dye Nile red was used, the structure of which is shown in figure 4.2. This choice is advantageous for the measurement in liquid crystals since the dye molecules orient along the director of the liquid crystal [88] and are consequently not expected to alter the immediate molecular neighbourhood of the liquid crystal matrix. For the used wavelength of $488 \mathrm{~nm}$ (see section 4.5), the dye has an absorption of around $50 \%$ of its maximum [88]. The quantum yield strongly depends on the embedding matrix but is around $30 \%$ [89].

\subsection{Sample preparation}

Liquid crystal films on substrates were prepared by spin-coating from a toluene solution. Spincoating in general is an established technique used to obtain thin films over a large central area of the sample. For this method, a small amount of either material in solution or pure liquid material is deposited on the substrate that is supposed to be coated. Then the sample is rotated with a given spinning rate so that a uniform equilibrium film is achieved at the center of the sample. In case the material is dissolved in another liquid, the solvent would evaporate and leave behind a film that is thinner than the original equilibrium film. In spin-coating the conditions that are used can be varied with respect to different parameters. Examples for this are the acceleration, the amount of material deposited or the ramp of the acceleration (for example using different intermediate spin speeds). However, it turns out that only the 
concentration in the solution and the final spin speed decide the thickness of the resulting deposited film as long as no significant amount of solvent has evaporated before [90].

For this work it was decided to keep all the parameters for spin-coating constant and only to vary the concentration of $8 \mathrm{CB}$ in toluene. For each coated substrate $20 \mathrm{ml}$ of the solution was deposited and a spin speed of 6000 RPM was used. For the diffusion measurements the dye molecules were dissolved in toluene with a concentration of around $10^{-9} \mathrm{~mol} / \mathrm{l}$. This leads to a concentration of dye molecules in the final film of around $10^{-7} \mathrm{~mol} / \mathrm{l}$. As substrates silicon wafers were used. They are known to provide complete wetting conditions for $8 \mathrm{CB}$ if the surface of the silicon is covered with silanol groups, the latter rendering the substrate hydrophilic [91].

The silicon wafer was cut into quadratic pieces of around $1 \mathrm{~cm}$ length prior to all treatment. Then they were cleaned first with ethanol to get rid of all crude dirt. The decisive step in cleaning is the use of piranha solution (60\% concentrated sulfuric acid, $40 \%$ hydrogen peroxide) at a temperature of around $70^{\circ} \mathrm{C}$. This solution is a strong oxidiser so that it will react with most of the organic materials on the substrate surface that would otherwise show as background in the fluorescence experiments. Also piranha solution reacts with the siloxanes on the surface to silanoles [92]. This is important to get a homogeneous and hydrophilic surface.

For the study of the structure formation on substrates, silicon wafers with native oxide were used (CrysTec). However, those substrates were not suitable for fluorescence experiments as we are always working with films not thicker than $20 \mathrm{~nm}$. If a fluorescent molecule is so close to the bulk silicon, the de-excitation is not done mainly via fluorescence but the fluorescence is quenched by energy transfer to the silicon [93]. For this reason silicon with thermally grown oxide of $100 \mathrm{~nm}$ thickness (Si-Mat) was used.

Contrary to the films on the substrates, for the free-standing films the controllability of the thickness is very low. The common technique is to produce the films by drawing the smectic material over a metal frame using a flat edge, in our case a flat spatula. This gives a freestanding film without controle over the number of layers obtained. Unlike sometimes stated in literature [40] it was not possible for any of the mesogens that we were studying to change the film thickness afterwards by simply using one movable end of the frame in order to increase the area of the film. In our case the thickness was always stabilised by material flowing into the film from the meniscus or vice versa upon decrease of the film area. Another technique to 

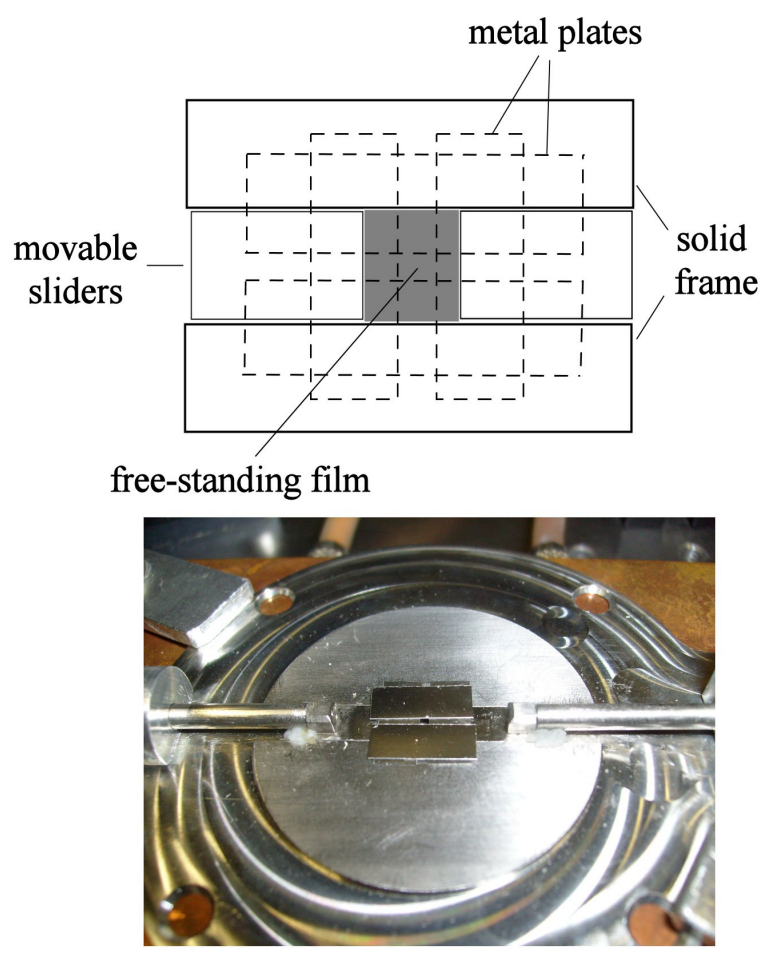

Figure 4.3: Schematic top view and photo of the sample holder for the free-standing films. The film (shown in grey) is spanned by moving outward the sliders. The thin metal plates over the film lead to a reduced temperature gradient around the sample. The temperature control is done from below.

decrease the film thickness is giving a thermal impulse [41]. This, however, could not be used as other priorities in the setup make the realisation of this method difficult (see chapter 4.5).

In order to dissolve the dye molecules in the liquid crystal matrix, the dye was first prepared as a solution of toluene with an appropriate concentration and then mixed with the liquid crystal. The mixture was kept at $70^{\circ} \mathrm{C}$ for around 24 hours in order to evaporate the toluene.

The temperature of the film was controlled using a Peltier element and a PID controller (McShane 5R7-002). This made it possible to control the temperature with a precision of around $0.1 \mathrm{~K}$ in a range between $0^{\circ} \mathrm{C}$ and $80^{\circ} \mathrm{C}$. For measurements at temperatures higher than $50^{\circ} \mathrm{C}$, thermal gradients induced convection in the film [94]. A thermal insulation was therefore constructed over the film with metal plates thermally connected to the frame. A schematic top view of the sample holder is shown in figure 4.3 together with a photo. 
Another problem in stabilising the film is that any air flow induces a flow also within the film. Thus, the film was placed in an air tight protection.

\subsection{Atomic force microscopy}

Atomic force microscopy (AFM) is a probe microscopy that is able to scan charged and uncharged surfaces with a resolution in the nanometer scale. Since its invention in 1986 [95], it has found auch a wide range of applications to all kinds of surface analysis, that nowadays only a short explanation is necessary in such a thesis. The concept depicted in figure 4.4 shows that the surface is scanned using a cantilever with a sharp tip of a size of a few nanometers. When the tip is approaching the sample, the cantilever gets deflected by a variety of forces [96]. This deflection is recorded by a laser beam that is reflected from the back side of the cantilever and then registered by a (usually 4-fold) photodiode. With the help of the photodiode the deflection is kept constant when moving the tip over the sample which then gives the surface topography as a reconstruction of the up and down movements of the cantilever.

Substantial improvement of AFM imaging was made by the invention of the tapping mode [97]. Here the tip oscillates around its resonance frequency. When approaching the sample, the amplitude is changed due to the interaction forces. The amplitude is used as the feedback parameter that is kept constant. The advantage of the tapping mode is that often in contact mode there is a meniscus formed between the tip and the sample because of the water film which is adsorbed on the surface under ambient conditions. This is avoided by the oscillations in tapping mode. Also it becomes possible to measure liquid samples [98] and consequently also thin films of liquid crystals [91] as well as bulk surfaces [99]. This was used in this thesis to measure surface structures that are too small to be resolved optically (see chapter 6.1.1).

For all the experiments, the AFM (Veeco diMultiMode) was employed in tapping mode for the imaging of the surface and in contact mode for the surface manipulation of thin films. Standard silicon cantilevers with a spring constant of either around 2 N/m (Olympus AC240TS) or around $42 \mathrm{~N} / \mathrm{m}$ (Olympus AC160-TS) were used. Also the AFM included a temperature control unit that enabled experiments above room temperature. If not stated otherwise, the experiments were conducted at room temperature, which was around $23^{\circ} \mathrm{C}$.

When measuring liquid crystal surfaces, it is important to keep the interation strength between the tip and the sample as low as possible in order to avoid removal of the soft surface structure. Therefore, one has to work on the one hand with a low free amplitude of the can- 


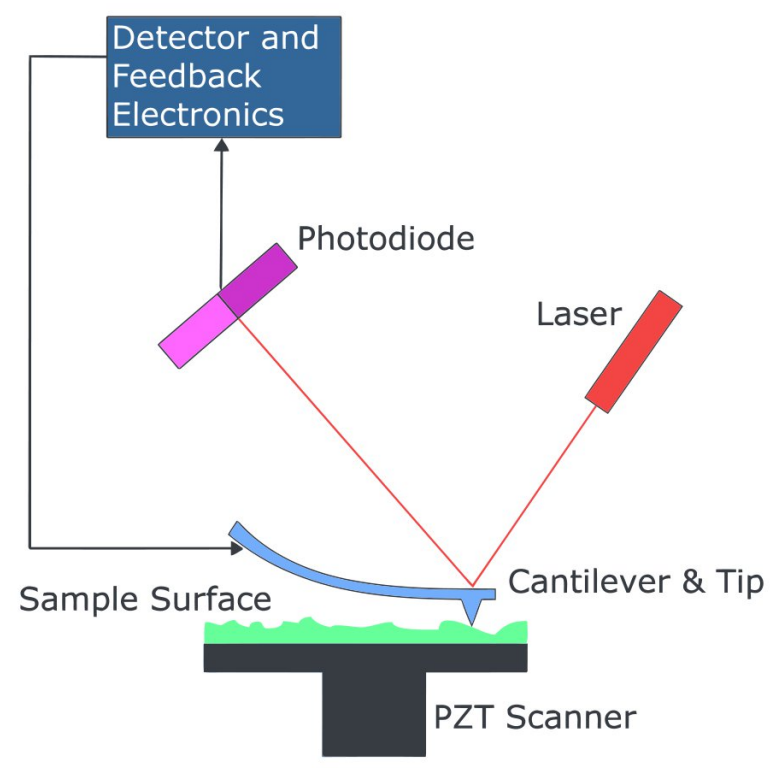

Figure 4.4: Schematic setup of an atomic force microscope $\left.\right|^{2}$ The interaction of the tip and the surface is registered by the changing reflection of a laser beam. Scanning the whole sample leads to a reconstruction of its surface topography.

tilever and on the other hand with a low damping. In pratice, for each tip the free amplitude was chosen as low as possible to still allow imaging of the surface. Typical amplitude setpoints were around $95 \%$ of the free amplitude.

\subsection{Ellipsometry}

Brewster angle ellipsometry was employed to measure the film thickness of the thin films on substrates. This method is very sensitive to slight changes of the surface properties [100]. It also enables one to study the formation of layers on molecular level in liquid crystals [101]. The basic concept and the relevant physical quantities are shown in figure 4.5. A laser beam $(\lambda=633 \mathrm{~nm})$ with equal components polarised parallel and perpendicular to the plane of incidence strikes the sample at an angle that is the Brewster angle for the bare substrate. As the measurements take place in air with the refractive index $n_{0}=1$, the Brewster angle is $\theta_{\mathrm{B}}=\arctan n_{2}$, with the refractive index of the substrate material $n_{2}$. Characteristic for this

\footnotetext{
${ }^{2}$ Image downloaded from public domain.
} 


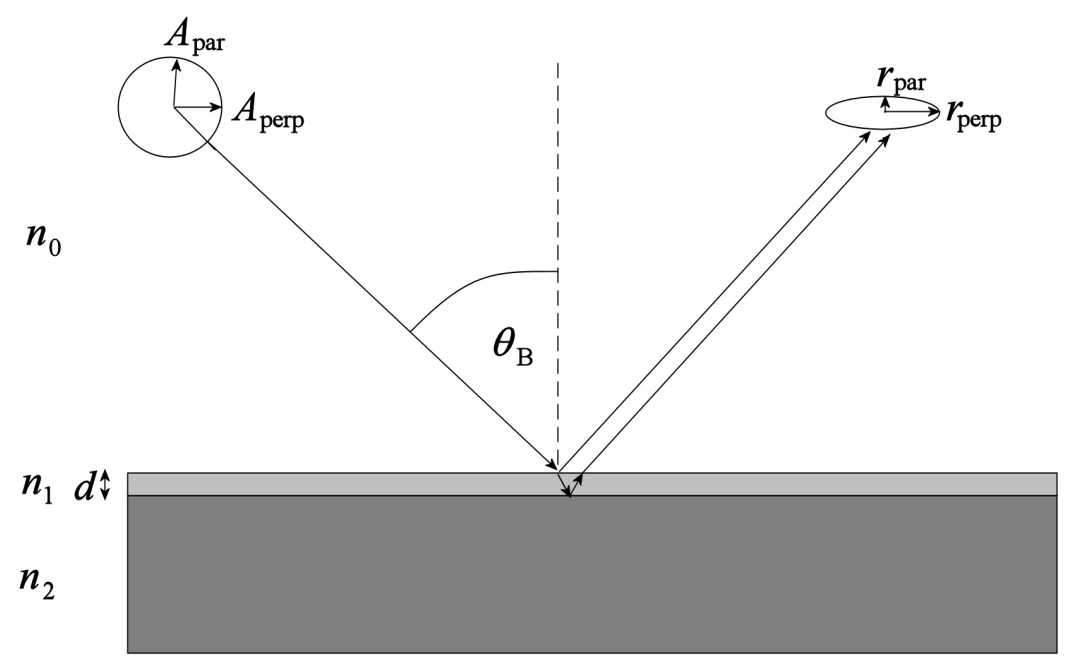

Figure 4.5: Measurement concept of Brewster angle ellipsometry: Originally circularly polarised light strikes a mirror at the Brewster angle $\theta_{\mathrm{B}}$. Depending on the thickness $d$ of the film on the substrate, different amplitudes of the light parallel and perpendicular to the plane of incidence are found.

angle is that the reflected light is linearly polarised perpendicular to the plane of incidence. In the presence of a film on the substrate, the reflected intensity is the interference of the reflections from the two interfaces. This changes the polarisation state of the reflected light with the amplitudes of the component of the light polarised parallel to the plane of incidence $r_{\text {par }}$ and perpendicular to it $r_{\text {perp }}$. Those values are measured and the ellipsometric quantities are calculated from it, namely the ellipsometric angles $\Delta$ and $\Psi$ defined by

$$
\frac{r_{\text {perp }}}{r_{\text {par }}}=\frac{\left|r_{\text {perp }}\right|}{\left|r_{\text {par }}\right|} e^{\mathrm{i}\left(\varphi_{\text {perp }}-\varphi_{\text {par }}\right)}=\tan \Psi e^{\mathrm{i} \Delta} .
$$

For the case of $\tan \Psi$ measured at the Brewster angle it is called the ellipticity coefficient $\bar{\rho}$. The angle of incidence is varied in a small range around the expected Brewster angle. The identification of $\bar{\rho}$ is defined as the minimum of the the angle dependence of $\tan \Psi$.

In principle, the ellipticity coefficient allows to calculate the thickness of the film when knowing the optical properties of the film. However, for birefringent materials the calculation of the thickness is not straightforward. Instead, a model of the orientation of the optical axis has to be fitted to the data [102]. The agreement between the model and the experiment 
supplies on the one hand the validation of the model and on the other hand the film thickness as a fit parameter.

The setup used for the thickness measurement is a phase modulated ellipsometer [103]. The polarisation is modulated with a frequency of $50 \mathrm{~Hz}$ between parallel and perpendicular polarisation of the incident and the reflected light. However, this method does not change the considerations done above for the general concept of ellipsometry using circularly polarised light.

\subsection{Single molecule setup}

A part of this doctoral thesis was the task to build up a wide field epifluorescent microscope that on the one hand would enable single molecule tracking and on the other hand includes an interferometer to measure the thickness of a free-standing film at a resolution better than one molecular layer. The reason why both had to be included in one setup is that the transport of a free-standing film between two separate setups might already change the film thickness. As the precise information about the film thickness is crucial for all diffusion measurements, an in situ possibility to measure both is required.

The schematic view of the setup is shown in figure 4.6. The microscope has flippable mirrors that enable switching between the fluorescence microscope and the interferometer. The fluorescence mode uses nearly parallel laser light of wavelength $488 \mathrm{~nm}$ (Laser: Sapphire 488 , Coherent) with a power of $10 \mathrm{~mW}$. This light is passed through a lens in order to make it converging to achieve a wider illuminated region on the sample. The light is reflected to the sample by a dichroic mirror (505 DCXR, AHF Analysetechnik) and illuminating the sample by a high magnification objective lens (MPLFLN100x/NA 0.9, Olympus). The spot of the illuminated region on the sample had a diameter of around $60 \mu \mathrm{m}$. The fluorescent light coming from the sample together with the reflected light is collected by the same objective lens and passes the dichroic mirror. As the reflected light has an intensity of some orders of magnitude higher than the fluorescent light, one needs to filter out the shorter wavelength. This is done with the help of two longpass filters (ET 510 LP, AHF Analysetechnik). It was found that the use of only one longpass filter was not sufficient to filter out all the reflected light and that the $S N R$ could be increased by using a second one. Finally, the fluorescent light is focused with an achromatic lens (Thorlabs, focal length $f=15 \mathrm{~mm}$ ) on the camera (Andor $\mathrm{iXon}^{\mathrm{EM}}+897$ ). The camera is an electron multiplying CCD-camera with a resolution 


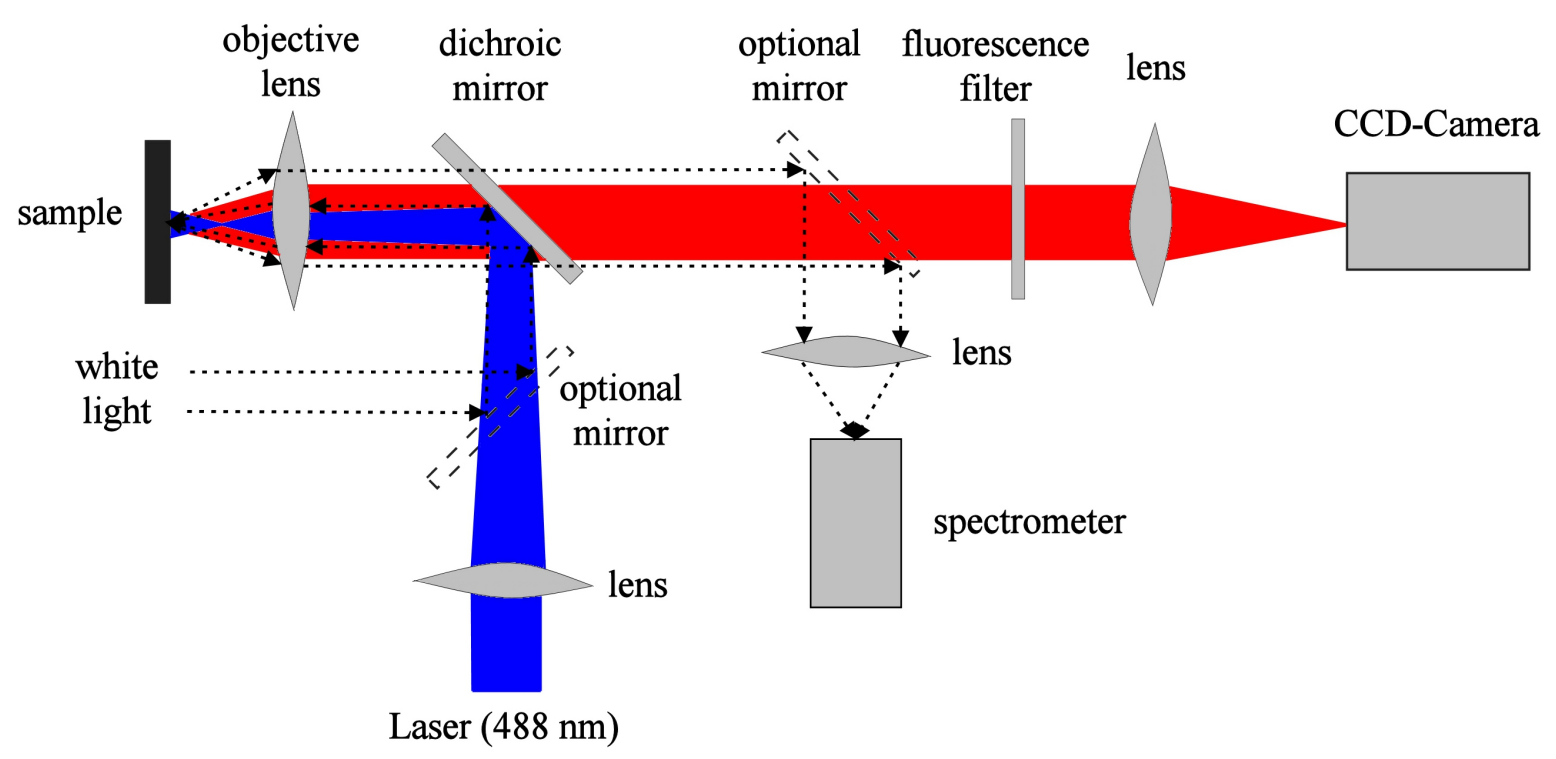

Figure 4.6: Schematic representation of the single molecule microscope drawn in solid lines with implemented optional switch to an interferometer shown with broken lines.

of maximum $512 \times 512$ pixels and a very high detection efficiency of over $90 \%$ in the whole range of wavelengths between 500 and $700 \mathrm{~nm}$ (supplier information). Also, the camera is cooled down to $-80^{\circ} \mathrm{C}$ to reduce the thermal noise of the camera. The camera was used with a framerate of $50 \mathrm{~Hz}$. The length of the captured movies was 50000 frames. A photo of the setup is shown in figure 4.7 .

The modus of the microscope can be changed by flipping two mirrors. This couples white light into the setup and at the same time blocks the laser light. The light source is a white light LED (CREE XP-G, LED-Tech) and has a nearly constant emission for wavelengths between 500 and $600 \mathrm{~nm}$ as shown in figure 4.8. The light reflected from the sample is collected and its spectrum is analysed by a UV/VIS-spectrometer (AvaSpec-2048-USB2, Avantes). It has to be said that with an ideal setup the measurement of the reflection spectrum would not be possible as the dichroic mirror in an ideal case would not reflect any light with a wavelength larger than $505 \mathrm{~nm}$. However, with the dichroic mirror in use one has a reflection of around $3 \%$. Using long exposure times of $200 \mathrm{~ms}$ and averaging over 40 measurements gives a strong enough signal to measure the thickness.

The identification of the trajectories is a non-trivial task as the dye molecules show blinking as nearly all kinds of single light emitters do [104]. This blinking takes place on all time 


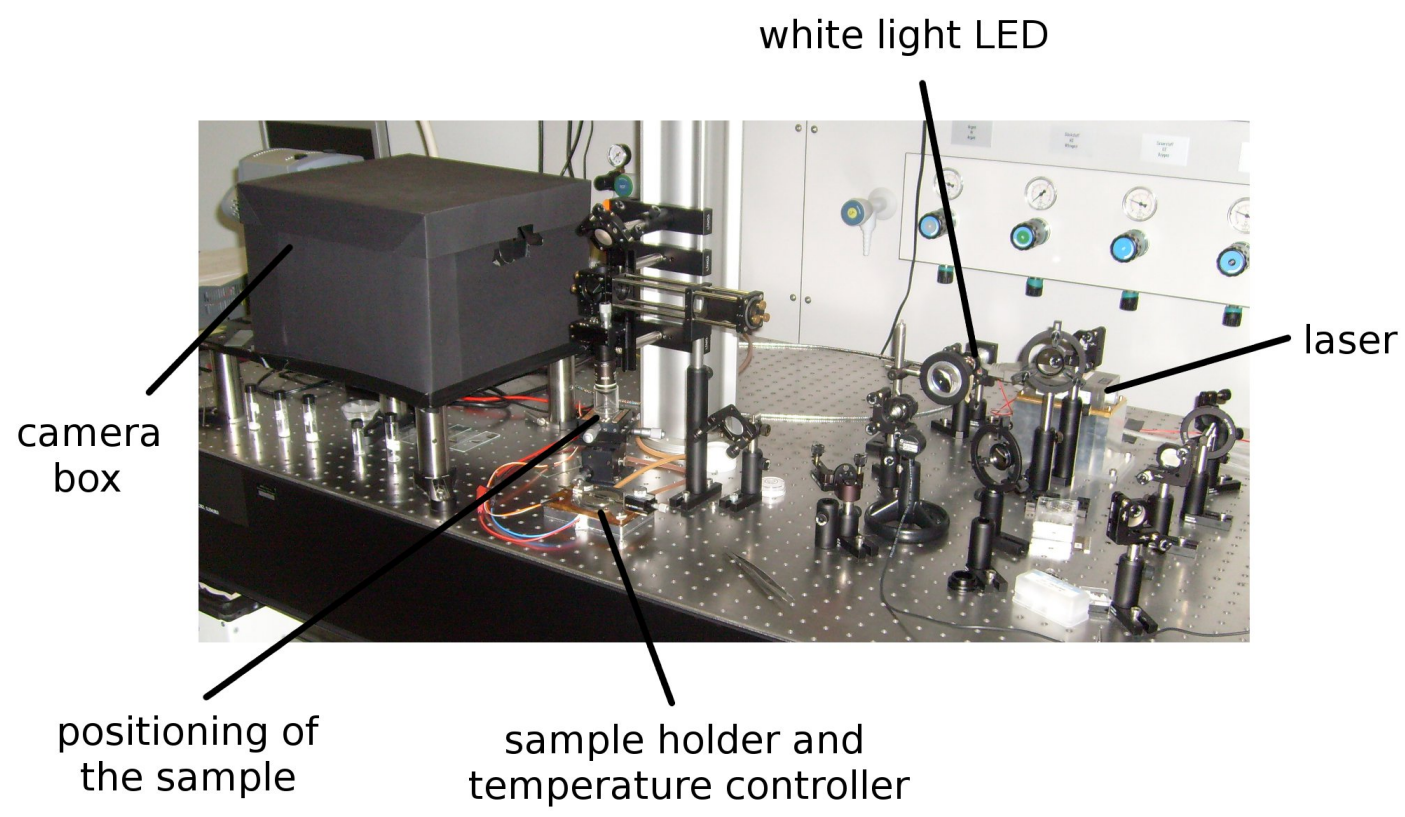

Figure 4.7: Photo of the single molecule microscope.

scales and leads to fluctuations in the light intensity due to the frequent short blinking times. Also, one observes the occurrence of dark states of molecules for a certain number of frames. This would interrupt the trajectory of the molecule every time a blinking in the range of the exposure time or longer happens. As explained in chapter 3.3.2, the statistical accuracy of a measured diffusion coefficient strongly increases with increasing trajectory length. The used software "Diffusion Simulation and Analysis" 3 tolerates the dark state of the molecule for two frames and still reconstructs the trajectory according to the highest probability of a molecule behaving according to the Fokker-Planck-equation [106]. For the analysis only trajectories with a length of at least 50 frames were used.

Additionally, it is possible to display the trajectories before the calculations of the diffusion coefficients using the software. This helps to eliminate trajectories that were evidently reconstructed in a wrong way, meaning assigning two different molecules to one trajectory. Examples for accepted and rejected trajectories are shown in figure 4.9. If this is not done, one tends to overestimate the average diffusion coefficient as wrong assignments usually lead to the calculation of faster diffusion. Also, without selection of the trajectories the distribution is not Gaussian anymore as displayed in figure 4.9 but would become a long tail distribution.

\footnotetext{
${ }^{3}$ Software written by Marion Heidernätsch [105] free under GNU General Public License version 3
} 


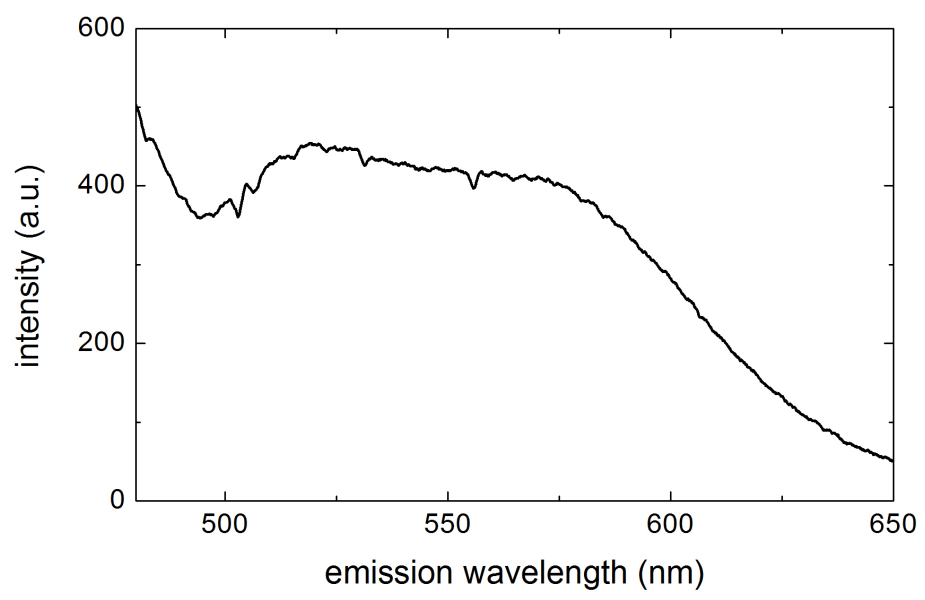

Figure 4.8: Spectrum of the white light source showing a nearly constant emission level in the range between 500 and $600 \mathrm{~nm}$.
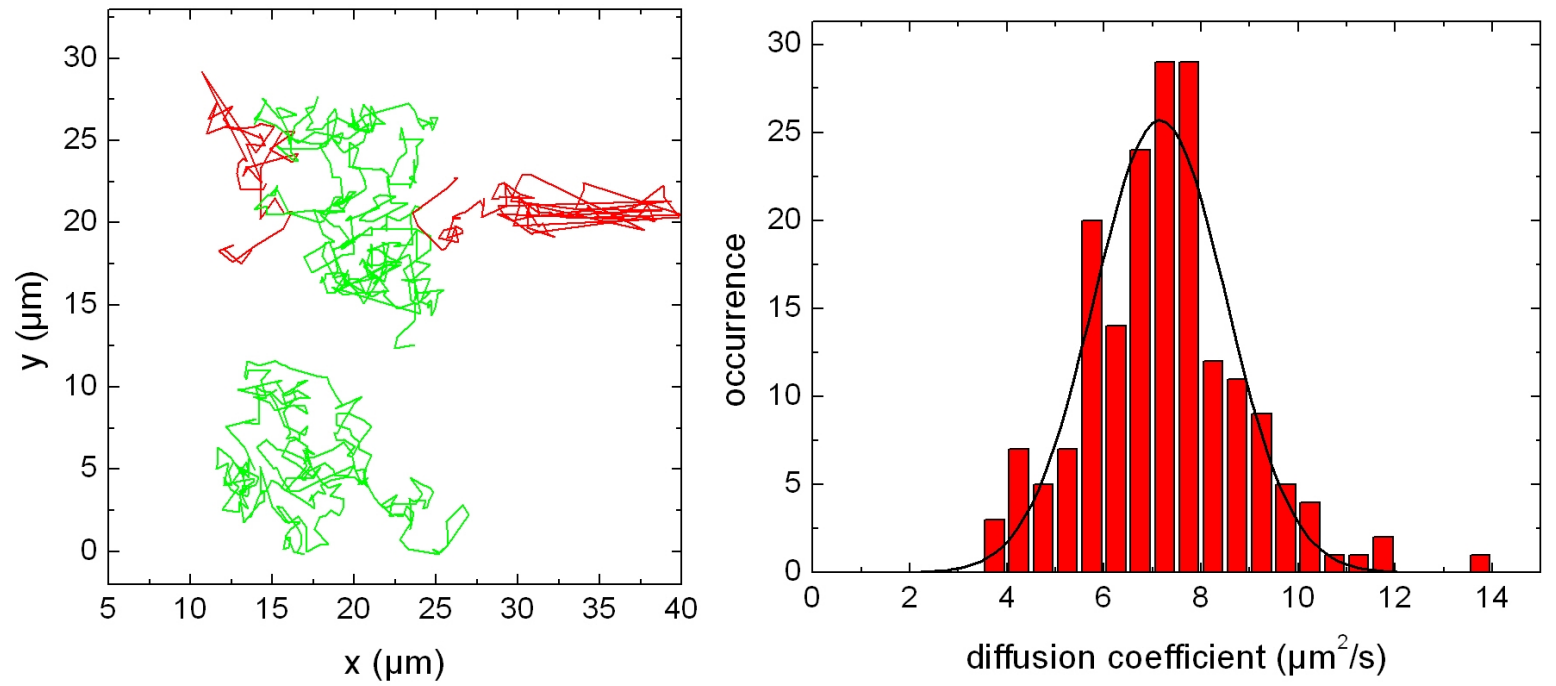

Figure 4.9: Left: Examples of accepted (green) and rejected (red) trajectories. The rejected trajectories appear to be constructed from two different molecules as the reconstructed trajectory jumps back and force. Right: Distribution obtained by the selected trajectories for the whole diffusion movie shows a Gaussian distribution in good approximation. 
In our calculations, the center of the Gaussian fit was used as an average of the diffusion coefficient. This helps to reduce the influence of outliers that might be dominant in the case of the calculation of the arithmetic mean. 


\section{Chapter 5}

\section{Diffusion in free-standing liquid crystal films}

As it was explained in chapter 2.5.2, free-standing smectic films show the unique feature that they can be prepared down to a thickness of only two layers without any supporting substrates. It can readily be assumed that a material with such an exclusive property can be different in its physical behaviour in many ways. For example, it was shown that particular surface phase transitions occur for single molecular layers [54]. In this thesis the focus will be put on the diffusional behaviour of free-standing films.

While other studies mainly probe the increasing influence of the solid interface when going to ultrathin films [107], our system allows us to measure thin film effects in the pure material as the surface is free. Hence, completely different properties are expected when moving to an increasingly two-dimensional diffusion by reducing the film thickness to the experimental minimum. This question shall be addressed in the first section of this chapter. The most obvious change is that the missing neighbouring molecules at the air interface would reduce the friction between the layers, thereby leading to a faster diffusion according to the StokesEinstein equation in form of equation (3.6). However, such descriptions arising from the assumption of a homogeneous medium might as well completely lose their validity when the properties are dominated by the processes at a molecular level.

Apart from the reduction of the dimensions of the sample, it is also possible to analyse the diffusion when approaching different kinds of phase transitions. Here, especially the first order phase transitions are expected to have a strong impact on the diffusion coefficient. The 
question, for which phase transition this expectation holds true, is the topic of the second section of this chapter.

The last section deals with free-standing films that are either axially or laterally inhomogeneous. The focus for these films is put on proving the possibility to distinguish between different regions of the film and on the behaviour of the molecules in the presence of phase boundaries.

However, before analysing the diffusion in free-standing films, it is necessary to have the exact information about the number of layers the film consists of. This was done by the analysis of the reflection spectrum of the film. The intensity $I$ of the reflected light is determined by the film thickness $d$, the refractive index $n$, and the wavelength $\lambda$. The interference between the light reflected at the upper and the lower side of the film determines the spectrum according to the equation [108]:

$$
\begin{aligned}
& I=A \frac{f \sin ^{2}\left(\frac{2 \pi D}{\lambda}\right)}{1+f \sin ^{2}\left(\frac{2 \pi D}{\lambda}\right)}, \\
& \text { with } f=\frac{\left(n^{2}-1\right)^{2}}{4 n^{2}} .
\end{aligned}
$$

Here, $D$ is the optical path length which is $n \cdot d$. As the liquid crystal is aligned homeotropically, the refractive index was assumed to be the ordinary index $n_{\mathrm{o}}$.

As the white light intensity was not exactly constant, the shown reflection intensities were always divided by the incident light power. Caused by the specific requirements of the setup, the white light had to be reflected by a dichroic mirror (see chapter 4.5). This changed the reflection spectrum of the samples. As a reference, for all the measurements the reflection spectrum of a mirror was recorded to normalise the spectra of the free-standing films.

The advantage when measuring the reflection spectra of free-standing films compared to films on substrates is that if the film thickness tends to zero the reflection signal also tends to zero. This means that there is practically no background signal of the reflection spectrum, which in turn offers the possibility to determine the film thickness with a precision better than one smectic layer using the absolute values of the reflection intensity. When producing films with a random number of layers, one finds the spectrum to show discrete absolute values. Also when the film thickness changes during the equilibration of the film, the spectrum changes in 
a)

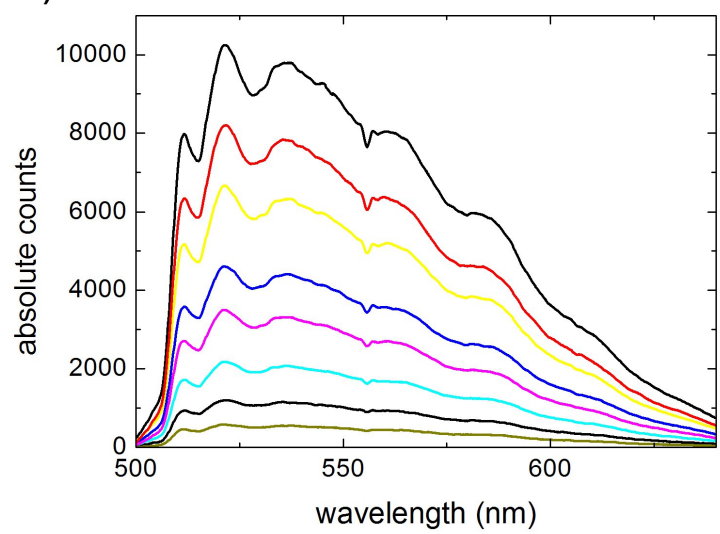

b)

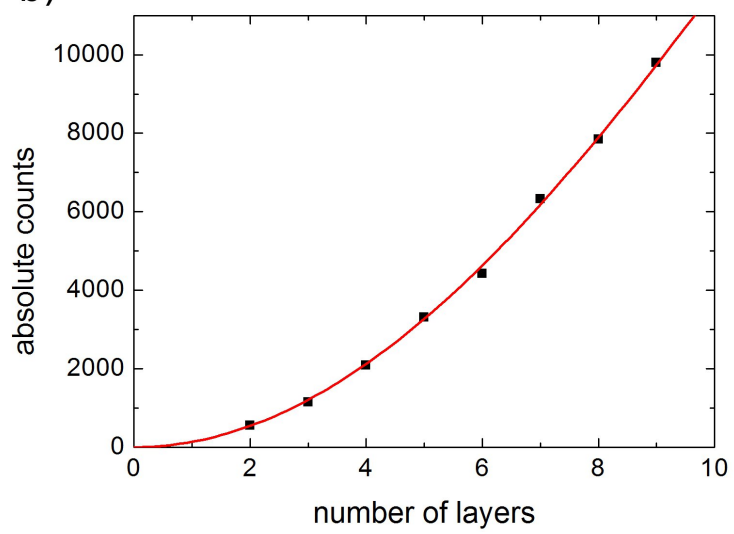

Figure 5.1: Determination of the film thickness: a) Discrete spectra observed for thin films of $8 \mathrm{CB}, \mathrm{b})$ Fits for the values obtained for the maximum at $535 \mathrm{~nm}$ using equation (5.1).

jumps, and never in a continuous manner. The spectra found for thin films of $8 \mathrm{CB}$ are shown in figure 5.1. The graph shows the spectra for an exposure time of $200 \mathrm{~ms}$ averaged over 40 frames. One can now check if the reflection changes according to equation (5.1). For this the reflectivity at the maximum of the graphs around $535 \mathrm{~nm}$ is plotted over the number of layers assuming that the minimum film thickness is 2 layers and the difference to the consecutive film thicknesses is exactly one layer. Using the known layer spacing of $3.16 \mathrm{~nm}$ [109], there is only one fit parameter left (the constant $A$ ). As depicted in figure 5.1, there is a good agreement with the experimental data. Also the fit curve has a maximum of around 37500 counts. This agrees with the observation that the maximum reflectivity is around 36000 counts for all the produced films. One can thus assume to have the full information about the number of layers for a given sample.

The spectra of $8 \mathrm{CB}$ can also be used to find the layer spacing for the other liquid crystals. The constant A in equation (5.1) is the same for all the materials. Hence, if one measures the dependence of the reflection intensity on the number of layers one gets the thickness of one layer. 

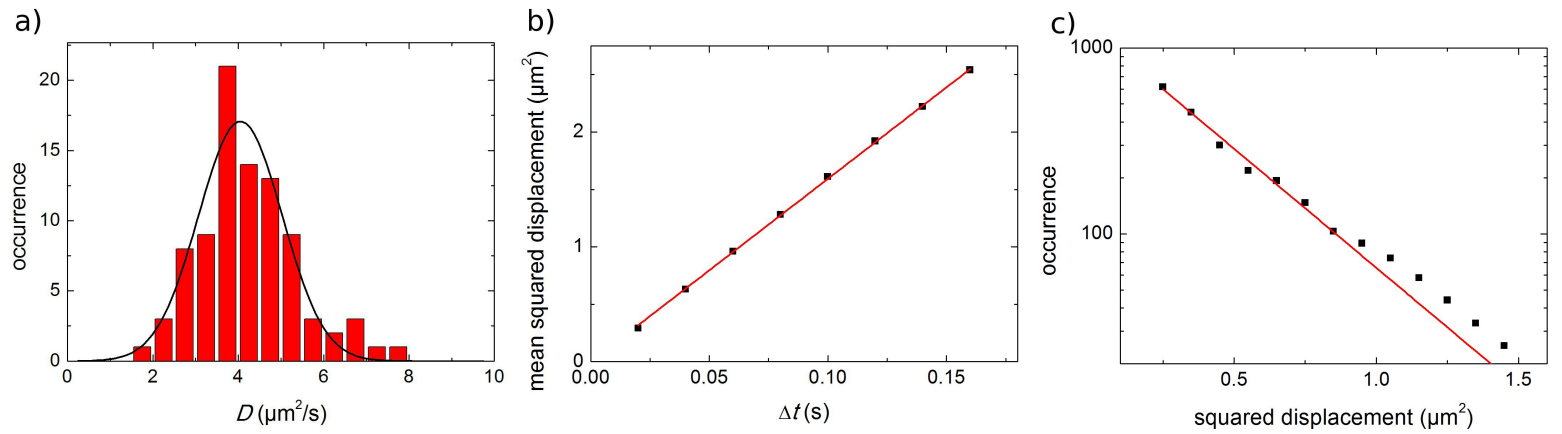

Figure 5.2: Comparison of different methods of calculating the diffusion coefficient for the example of an 8CB film consisting of 7 layers: a) After calculating $D$ for all the individual trajectories, the average $D$ is found as the center of the distribution. b) Plotting the mean squared displacements over the time lag gives $D$ according to equation (3.4) from the slope. c) The probability of finding a molecule being displaced by $(\Delta r)^{2}$.

\subsection{Faster diffusion in thinner films}

\subsubsection{Experimental results for different compounds in the smectic A phase}

In this section it shall be analysed how the reduction of the film thickness in a free-standing film affects the diffusion coefficient $D$. As explained in chapter 4.2, there was no controlled way to create films with a defined thickness. The usual procedure was to prepare a free standing film with an arbitrary film thickness and measure the number of layers with the help of the interferometer as explained in the beginning of this chapter. After the diffusion measurement, the film thickness was measured again to check that the film thickness did not change during the measurement time. Obviously with this method one cannot completely exclude the possibility that the film thickness changed during the measurement and changed back to the original film thickness. However, such a process can be considered improbable as the film thickness is highly stable after the equilibration of the film.

A typical equilibration time of the film was around 30 minutes. However, it was always possible to judge by eye if a film has already equilibrated, as in video microscopy even a slow flow is easily visible as a preferred direction of movement of the molecules. It was argued in chapter 4.5 that the center of the distribution of the diffusion coefficients is preferable for the calculation of the diffusion coefficient. Nevertheless, it should be tested to which extent there is an agreement between the different methods of calculation. Figure 5.2 shows three ways to calculate the diffusion coefficient for the example of an $8 \mathrm{CB}$ film with 7 layers. Graph a) 
shows the calculation as described by calculating the diffusion coefficients for all the identified trajectories. The center of the Gaussian fit was taken as the average diffusion coefficient, which in this case is $D_{\text {Gauss }}=4.05 \mu \mathrm{m}^{2} / \mathrm{s}$. Secondly, one can plot the mean squared displacement for the different time lags $\Delta t$ which is shown in b). The diffusion coefficient can be calculated from the slope of the linear fit according to equation (3.4), and one finds $D_{\mathrm{msd}}=3.98 \mu \mathrm{m}^{2} / \mathrm{s}$. Thirdly, one can use the probability distribution for the diffusion from equation (3.5) which is shown in c). It is used that the probability for the squared displacements of one step scales exponentially. Thus, the exponential decay (squared) length of the fit gives the diffusion coefficient. One obtains $D_{\text {prob }}=4.24 \mu \mathrm{m}^{2} / \mathrm{s}$.

There is a very good agreement between the calculation by the mean squared displacement and the distribution of diffusion coefficients. This, however, is not surprising as both methods practically use the same data. Just the averaging takes place in a different way. For the calculation via the probability distribution, one finds a value differing by $5 \%$ with respect to the other methods. For larger steps one also finds a slight systematic deviation from the fitted curve.

The error for the calculation with the Gaussian distribution can be estimated as the standard error of the fit plus the statistical error. The latter is the standard deviation of the mean, which means if one has $l$ measured values that have a standard deviation of $\sigma$, one has a statistical error of

$$
\sigma_{\text {mean }}=\frac{\sigma}{\sqrt{l}} .
$$

For our example, the diffusion coefficient calculated from the center of the Gaussian distribution would be $D=4.05 \pm 0.27 \mu \mathrm{m}^{2} / \mathrm{s}$. In this way the error bars for all the following measurement were calculated. Hence, the value obtained using the probability distribution is still within the error.

One can also see in figure $5.2 \mathrm{~b}$ ) that the mean squared displacement scales linearly with time. Thus, the molecules show normal diffusion. This, of course, is expected for diffusion in a free-standing film, where there are no obstacles hindering the Brownian motion of the molecules.

It should be mentioned here that the method of single molecule tracking has an inherent tendency to underestimate the measured diffusion coefficients. The reason simply is that during the exposure time of the camera the molecule moves significantly. Therefore, one only measures the average position of the molecule during the exposure and not the momentary position at a given time. One can correct the values knowing the exposure time and the frame 


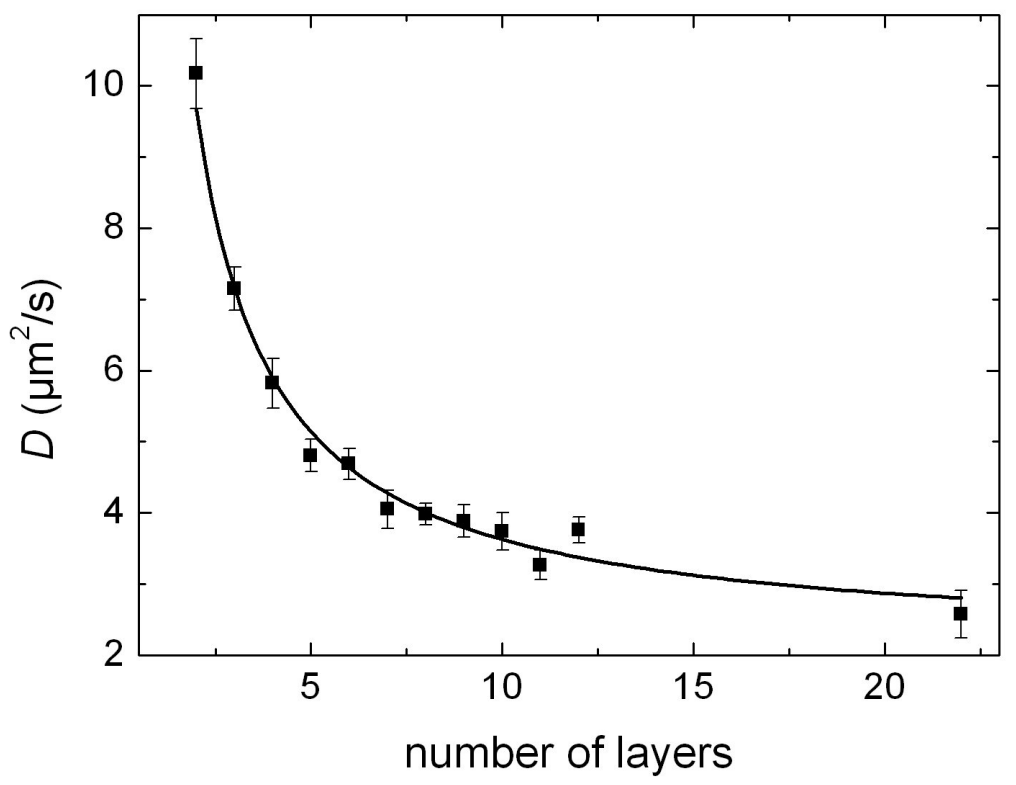

Figure 5.3: Film thickness dependence of diffusion in thin free-standing films of 8CB varying the film thickness between 2 and 22 layers. The fit function shown as solid line is explained in the text.

time (which is the exposure time plus the readout time of the camera) of the movies [110]. However, the employed method does not measure self-diffusion of the liquid crystal but only the diffusion of the probe molecules. Therefore, the absolute values of the diffusion are of minor importance for our analysis. The focus is purely on the comparison of diffusion coefficients for different free-standing films. Thus, the values presented in the following are the uncorrected ones.

One can now look at the changes of the diffusion in $8 \mathrm{CB}$ when reducing the film thickness of the free-standing films down the experimental limit of two layers. The obtained values are shown in figure 5.3. There is a remarkable increase of the diffusion coefficient for the observed film thicknesses between 22 and 2 smectic layers by around a factor of four. This effect was observed in a similar way by Bechhoefer et al. [43] using fluorescence recovery after photobleaching. However, in that work the concentration of the dye molecules was at the saturation limit in order to get a strong enough signal. Also the dye molecule used for that study did not orient along the director orientation of $8 \mathrm{CB}$ and might cause a disturbance of the local director field of the liquid crystal molecules. This leads to artifacts in the measurements 

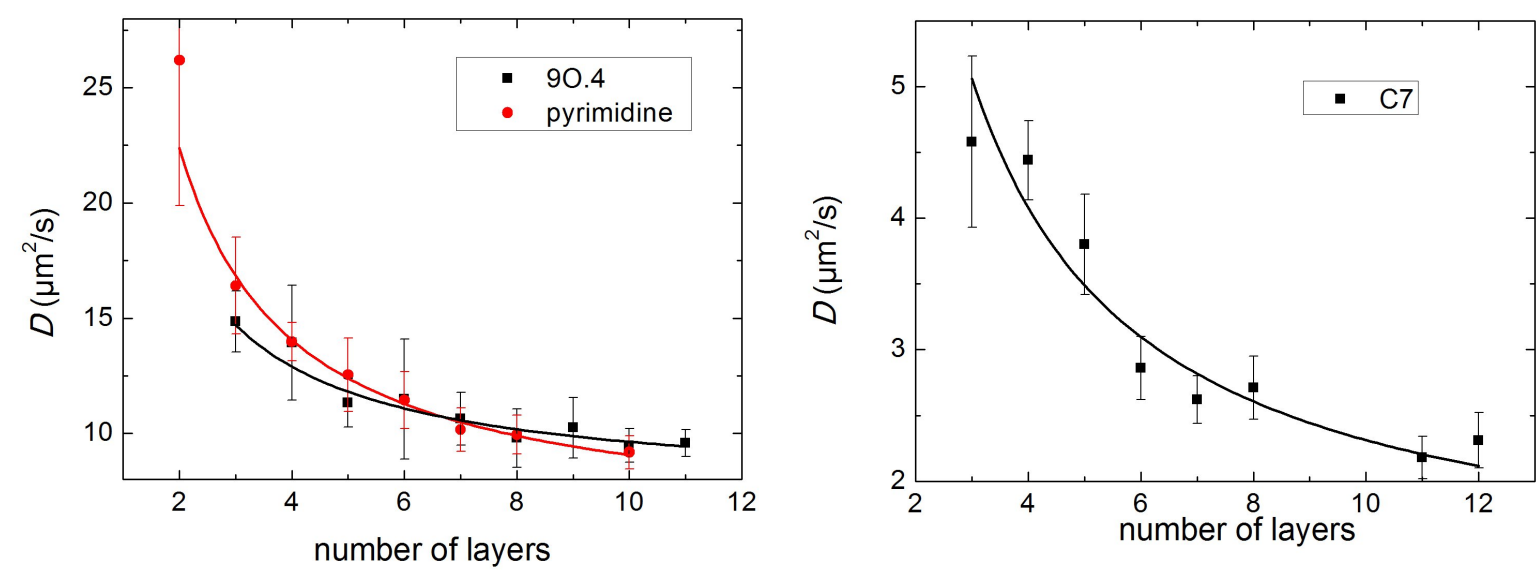

Figure 5.4: Film thickness dependence of diffusion in thin free-standing films of pyrimidine, 9 O.4 (both on the left), and C7 (right). The solid lines are fits using equation (5.4).

like an extraordinarily small value for the case of three layers. For our measurement no such exception could be found.

The fit function drawn in figure 5.3 is

$$
D=D_{\infty}\left(1+\frac{A}{n}\right)
$$

with $n$ being the number of layers, $A$ a dimensionless scaling parameter, and $D_{\infty}$ the bulk diffusion coefficient. The extent to which this equation can be explained by theoretical approaches will be given in section 5.1 .2 . So far it shall just be stated that this equation apparently gives a good fit to the data.

In order to test if the described functional dependence can be generalised, the film thickness dependence of the diffusion coefficient was measured for the mesogens pyrimidine, 90.4, and C7. The results for a smaller span of different film thicknesses are shown in figure 5.4. Unlike $8 \mathrm{CB}$, the above mesogens possess smectic layers constituted of molecular monolayers and not of double layers. All compounds exhibit several different smectic phases. However, the measurements were all conducted in the smectic A phase.

For the compounds 90.4 and C7, a measurement of only two molecular layers was not possible: The films of this thickness could be created, but were not stable for a long enough time to allow diffusion measurements. For pyrimidine the film was stable but the diffusion was very fast for the tracking of the molecules with the used setup. This data point was calculated 
out of only 16 reconstructed trajectories. Because of this, the value is of little reliability and contains a large error.

Generally all the shown dependencies of the diffusion coefficient seem to agree with the functional dependence from equation (5.4) as depicted by the fit functions. Still for C7 the scatter around the solid line is significant and no final statement can be made. The parameters $D_{\infty}$ and $A$ for the different mesogens are shown in table 5.1

\begin{tabular}{|c|c|c|}
\hline mesogen & $D_{\infty}\left(\mu \mathrm{m}^{2} / \mathrm{s}\right)$ & $\mathrm{A}$ \\
\hline \hline 8CB & 2.1 & 7.1 \\
\hline pyrimidine & 5.7 & 5.8 \\
\hline 90.4 & 7.5 & 2.9 \\
\hline $\mathrm{C} 7$ & 1.1 & 10.4 \\
\hline
\end{tabular}

Table 5.1: Scaling coefficients of the film thickness dependence of diffusion for 4 different mesogens

The comparison of the different values of $D_{\infty}$ does not contain reasonable information as it only reflects different mobilities in different bulk liquid crystals. More important is how strong the increase is when reducing the film thickness. This is reflected by the value of $A$. The scaling turns out to vary a lot between the different materials. Even neglecting the value of $\mathrm{C} 7$ because of the big scatter in the data, one still gets a difference of more than a factor of two for the different liquid crystals. To directly compare the relative change in the diffusion coefficient one can normalise the data for all the materials by dividing by $D_{\infty}$. This direct comparison is shown in figure 5.5. It would also be possible to scale it with the thickness of the film instead of the number of layers. However, it seems more appropriate to take the number of layers as the controlled variable as the physical properties are expected to change layer by layer and not with changing absolute value of the thickness.

It was, hence, shown that the strong increase in the mobility of the liquid crystal molecules upon reducing the film thickness is a common feature of free-standing films in the smectic A phase. Regardless of the mesogen, a drastic increase of the diffusion coefficient is observed. However, the absolute numbers of the scaling parameters are quite different as can be seen in figure 5.5. It would be useful to find out which properties of the mesogens decide about the scaling. Unfortunately, with the presented data this does not seem to be feasible. For example comparing the two most reliable data sets of $8 \mathrm{CB}$ and pyrimidine one finds a similar scaling 


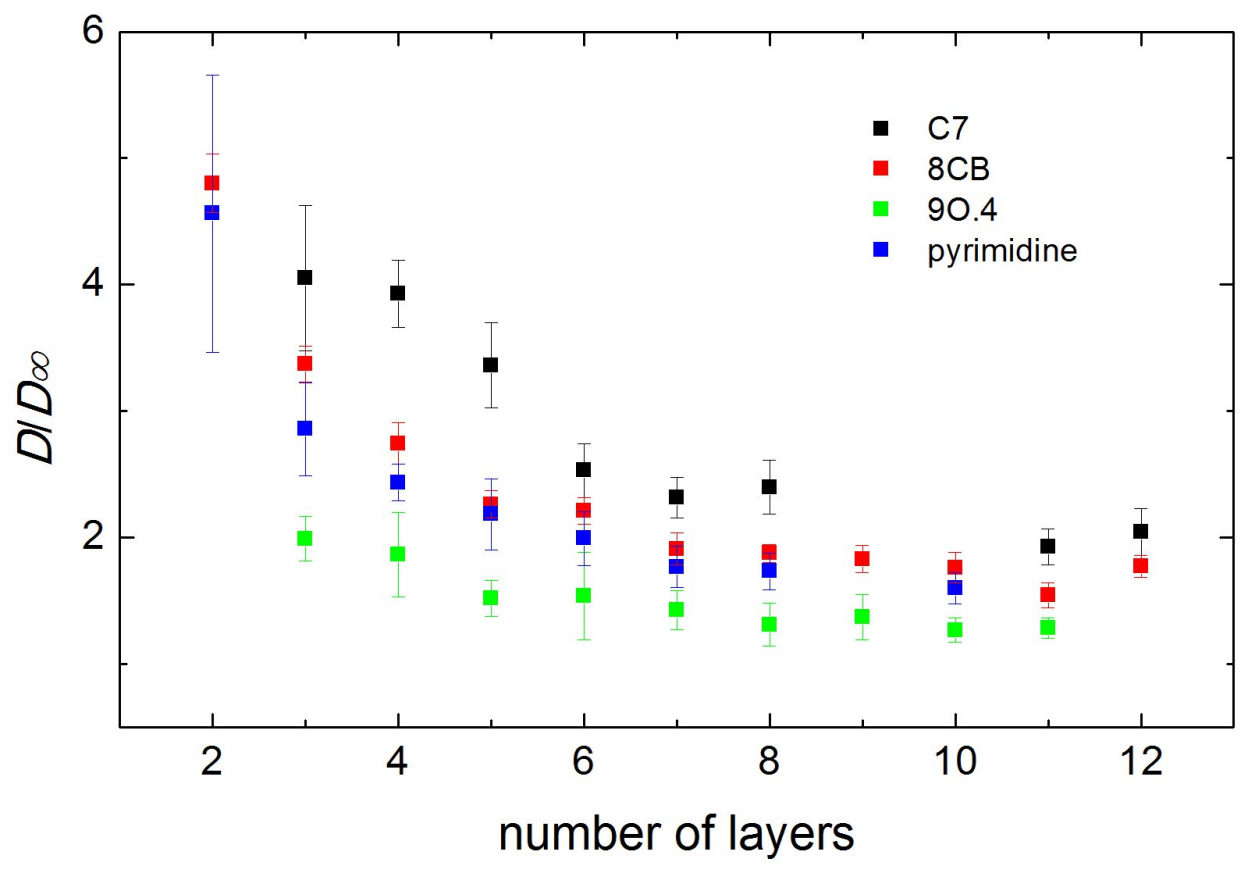

Figure 5.5: Film thickness dependence of the diffusion coefficients normalised by the bulk value $D_{\infty}$ for all the tested mesogens.

for two very different kinds of molecules. While $8 \mathrm{CB}$ has a large dipole moment and forms smectic layers consisting of molecular bilayers, pyrimidine has a small dipole moment and forms smectic layers consisting of monolayers. Also, no correlation with the length of the molecules (which is related to the aspect ratio) can be found.

Nevertheless, this strong increase can hardly be understood in terms of the classical diffusion theory. When using the Stokes-Einstein equation in the form of equation (3.6), the increase of the diffusion coefficient by a factor of four (e.g. for a two layer film of 8CB) would mean a decrease of the friction to one quarter of the original one. This cannot be justified simply by the absence of the molecules on top, as intuition would tell that the main part of friction must be caused by the molecules in the direction of motion. Also the diffusion is expected to be inversely proportional to the viscosity of the film following equation (3.7). The viscosity, however, was found to be slightly increasing for $8 \mathrm{CB}$ when reducing the film thickness of the free-standing film [111]. 


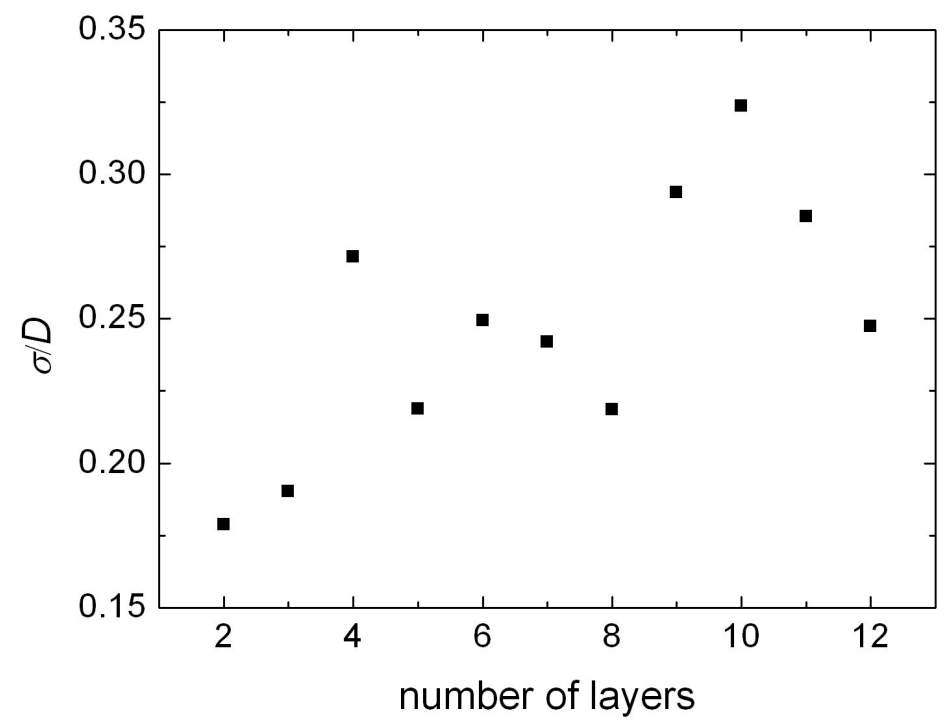

Figure 5.6: Standard deviation of the diffusion coefficients divided by the average diffusion coefficient of $8 \mathrm{CB}$.

Finally, it shall be tested if an influence of individual layers of the film can be identified in the diffusion data or if the molecules average over the entire film during the measurement time of one trajectory. For this, the standard deviation of the measurements for different numbers of layers shall be analysed. More specifically, the ratio of the standard deviation $\sigma$ divided by the average diffusion coefficient shall be considered for the case of $8 \mathrm{CB}$. The results depending on the number of layers are shown in figure 5.6. This value - which is the inverse of what is called the quality factor of a distribution - is known to be only dependent on the trajectory length for a homogeneously diffusing system [78]. However, despite the large scatter of the data, an overall increase of this value might be stated. This would be understandable in the way that in a film consisting of only two layers the diffusion coefficient is the same in the whole film; in case of more than two layers there are multiple diffusion coefficients that might be measured for different positions of the film. However, the dimensions of the films are only around 40 nanometers for the thickest considered film. For each trajectory, the molecules travelled several microns. The molecules are not expected to stay inside one smectic layer for so long to make a difference between two trajectories in the same film.

To get further insight into a possible distribution of diffusion coefficients, the distribution of single steps of the tracked molecules (defined by the time steps of the camera) shall be considered. For the case of the presence of a single diffusion coefficient, the distribution is given 


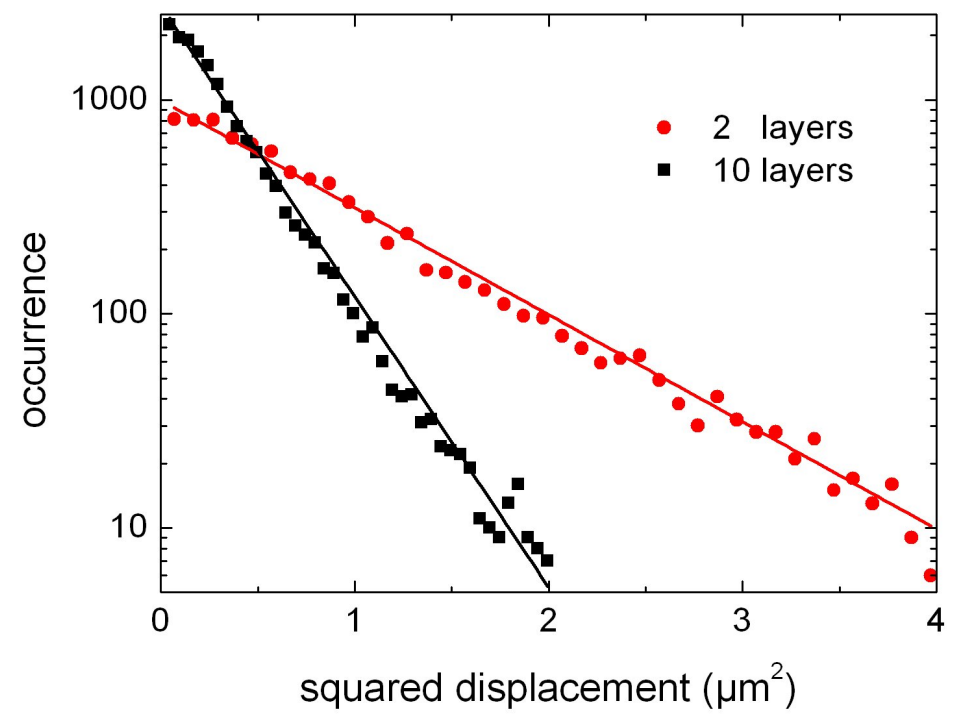

Figure 5.7: Distribution of squared displacements for a film of two and ten layers of 8CB. The fit functions are simple exponential decays according to equation 3.5 .

by equation (3.5). To find out if there are multiple diffusion coefficients visible in the thicker films, the validity of this equation for our samples shall be analysed both for the smallest and the largest value of $\sigma / D$, which are two and ten layers respectively. The comparison is shown in figure 5.7. Both distributions can be fitted very well with the assumption of only one diffusion coefficient present in the sample, as shown by the solid lines in the diagram. There is no indication that for the film of ten layers there is also a measurable part of the steps in the order of the steps found for the film consisting of two layers. The reason is that already for this one step (corresponding to a time lag of $20 \mathrm{~ms}$ ) the molecules travel a lateral distance of more than $300 \mathrm{~nm}$ which is one order of magnitude larger than the axial dimensions of the film. As only one diffusion coefficient is observed, the diffusion within the smectic layers does not appear to be strongly preferred compared to the diffusion perpendicular to the layers.

The distribution of the squared displacements analyses time scales that are around two orders of magnitude smaller in time compared to the full trajectory. For this reason the absence of any sign of heterogeneous diffusion observable in figure 5.7 means that the broader distribution of the diffusion coefficient shown in figure 5.6 is not caused by the presence of multiple diffusion coefficients in the sample resolvable with our method. 


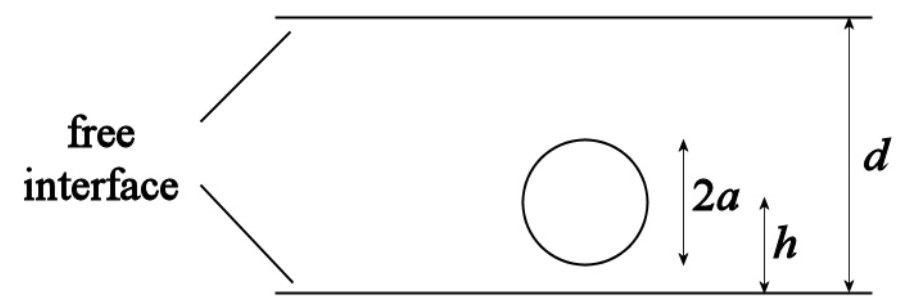

Figure 5.8: Definition of the values for the calculation of the increase in diffusion. A spherical particle of radius $a$ is at a distance of $h$ from the air interface. The thickness of the free-standing film is $d$.

\subsubsection{Theoretical considerations of the diffusion in thin free-standing films}

We have seen in the previous section that the dependence of the diffusion coefficient on the number of smectic layers can be written in the form

$$
D=D_{\infty}\left(1+\frac{A}{n}\right)
$$

regardless of the material used. It shall now be considered if this behaviour can be explained with theoretical calculation for the diffusion close to free surfaces. As an approach one can use the continuum theory of a diffusing spherical particle close to a free interface [112]. Considering only the diffusion perpendicular to the director $D_{\perp}, 1$ one finds:

$$
D=D_{\infty}\left(1+\frac{3 a}{8 h}\right)
$$

for a spherical particle of radius $a$ at a distance $h$ to one free (perfectly slipping) interface. For a free-standing film one has two free interfaces as represented in figure 5.8. There is an identical influence from both interfaces, so that the local diffusion coefficient in a film of thickness $d$ would be

$$
D=D_{\infty}\left(1+\frac{3 a}{8 h}+\frac{3 a}{8(d-h)}\right)
$$

\footnotetext{
${ }^{1}$ Note that in the cited reference the notation is different. There $D_{\perp}$ stands for diffusion perpendicular to the surface while here it is used for the diffusion perpendicular to the director.
} 
The total time of a reconstructed trajectory is at least one second. This is considerably larger than the assumed mean first passage time which would be in the order of few microseconds for a film of some tens of nanometres thickness [113]. Therefore, one can assume that a diffusing molecule explores all the different axial regions of the film. Hence, in order to calculate the consequences of this continuum approach, one has to average over the whole film. Also the molecules should always be completely immersed in the film, which gives the boundaries of the averaging from the dimensions of the molecule and the film:

$$
\bar{D}=\frac{1}{d-2 r} D_{\infty} \int_{r}^{d-r}\left[1+\frac{3 a}{8 h}+\frac{3 a}{8(d-h)}\right] \mathrm{d} h .
$$

The lower boundary of the integral $r$ is given as half of the long axis of the Nile red molecule. The prefactor comes from the normalisation of the integral. In this interpretation of the model, $a$ would be the hydrodynamic radius of the molecule. As the dye molecule was chosen in a way that it aligns along the director, there should be no big solvation effect in the liquid crystal matrix. However, the effect of the model under the assumption of no solvation is nearly negligible compared to the observed effect. For this reason the calculation is done under the assumption of a hydrodynamic radius larger than the molecular dimensions.

For the integration of equation (5.8) the first term is trivial. The second and the third term give the same contribution as both describe the influence of one free surface. As result one finds

$$
\bar{D}=D_{\infty}\left[1+\frac{3}{4} \frac{a}{d-2 r} \ln \left(\frac{d}{r}-1\right)\right] .
$$

Finally, setting the radius to $r=0.7 \mathrm{~nm}$, using the layer spacing of $8 \mathrm{CB}$ of $3.16 \mathrm{~nm}$, and defining the bulk diffusion coefficient as the value obtained from the fit in figure 5.3 to be $D_{\infty}=2.12 \mu \mathrm{m}^{2} / \mathrm{s}$, one has the hydrodynamic radius as the sole fit parameter. The obtained theoretical curve for the increased diffusion is shown in figure 5.9 .

It is obvious that this fit is worse than the one in figure 5.3. Also the necessary hydrodynamic radius would be around $9 \mathrm{~nm}$. This is more than 10 times the molecular radius which appears unrealistically large. Also giving the molecular radius as a free parameter in order to account for the possibility of molecular folding does not improve the fit. When using realistic restrictions for the fit paramters $-r$ being between half of the short axis and the long axis of the Nile red molecule, and $a$ being no larger than three times half of the long axis - lead to a clear underestimation of the real effect. Hence, this model cannot deliver an appropriate description of the observed increase in diffusion coefficient for thinner films. 


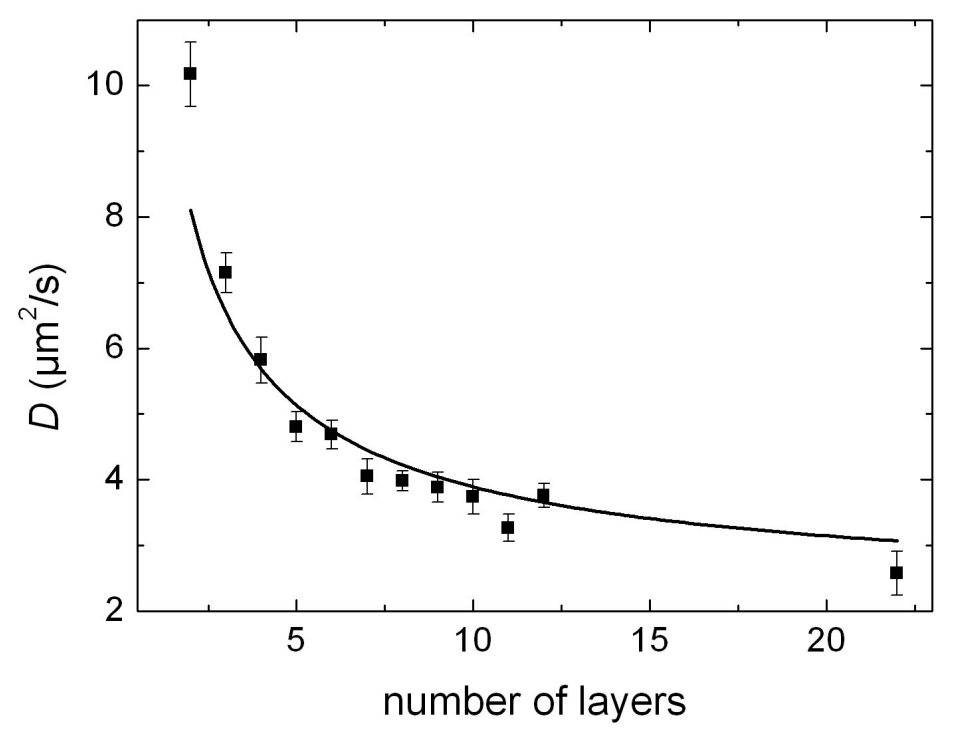

Figure 5.9: The solid line shows the theoretical curve describing the increase at a perfectly slipping surface according to equation 5.9. The data points are the diffusion coefficient in $8 \mathrm{CB}$ films identical to the ones shown in figure 5.3 .

Another approach to explain the increase of the diffusion in the scaling of equation 5.4 is using the Stokes-Einstein relation in the form

$$
D=\mu k_{\mathrm{B}} T
$$

with the mobility $\mu$. Now one can assume the existence of a hydrodynamic radius $a$ again. Then one can write a Taylor expansion for the mobility as [43]

$$
\mu=\mu_{\infty}\left[1+\frac{a}{2 h}+O\left(\frac{a}{2 h}\right)^{2}\right]
$$

with the distance from the interface $h$. The linear approximation leads exactly to the functional dependence for the diffusion coefficient from equation (5.4). This, however, seems more like an a posteriori argument as there is no reason to assume that $\left(\frac{a}{2 h}\right)$ would be small so that the approximation would be good. Quite contrary, the fit in figure 5.3 would lead to a hydrodynamic radius of more than $40 \mathrm{~nm}$. First of all this is unrealistically high, and secondly there is no reason to neglect the higher orders in the series expansion in powers of $a / 2 h$ that was used. 
Furthermore, theoretical considerations concerning the mobility in a membrane [114] were applied to free-standing liquid crystal films [115]. This, however, is not applicable to our system: The dye molecules are always completely immersed in the film while the model assumes objects that have larger dimensions than the film itself.

Possibly the most promising approach to explain the faster diffusion is the mode coupling theory. The idea behind it is that the collective hydrodynamic modes are sensibly influenced by geometrical confinements [116]. In the case of perfectly slipping boundaries this might lead to an increase in diffusion coefficient [117]. However, to the author's knowledge there is no theory existing so far that would be suitable for the case of free-standing films.

In summary, the existing models are not able to explain the observed increase of the diffusion coefficient in this magnitude that was found. The assumption of a hydrodynamic radius of the dissolved dye molecules leads to unsatisfactory results. One can thus conclude that the observed effect is not an artifact of the hydrodynamic radius around the tracer molecules but a consequence of the faster motion of the liquid crystal molecules themselves.

\subsubsection{Simulation of the self-diffusion in free-standing films}

As we have seen the previous section, there is no satisfying analytical description of such a strong increase in the diffusion coefficient. However, molecular dynamics simulations are well-suited to model the experimental system in a useful way. First of all the small dimensions of the sample limit the number of molecules that need to be simulated. Secondly, the distance to the long ends of the film is huge compared to the film thickness, thus giving a good justification for the use of periodic boundary conditions.

Such simulations done by Marco G. Mazza using a Gay-Berne-Kihara model [118]. These simulations show an increase of the diffusion coefficient similar to the presented experiments as depicted in figure 5.10. In spite of the different scaling factor $A$ there is a general agreement that the diffusion is strongly enhanced. This proves that the experiments are actually not showing an artifact of the diffusion measurement using tracer molecules but represent a real change in the mobility of the liquid crystal molecules.

The presented data only gives the first hint that simulations of the studied system is possible and that the results agree with the experiments. In the future this will allow for the analysis of the diffusion in individual layers of the film. This will also give some better insight into the molecular processes that lead to this observed strong increase in the diffusion coefficient. 


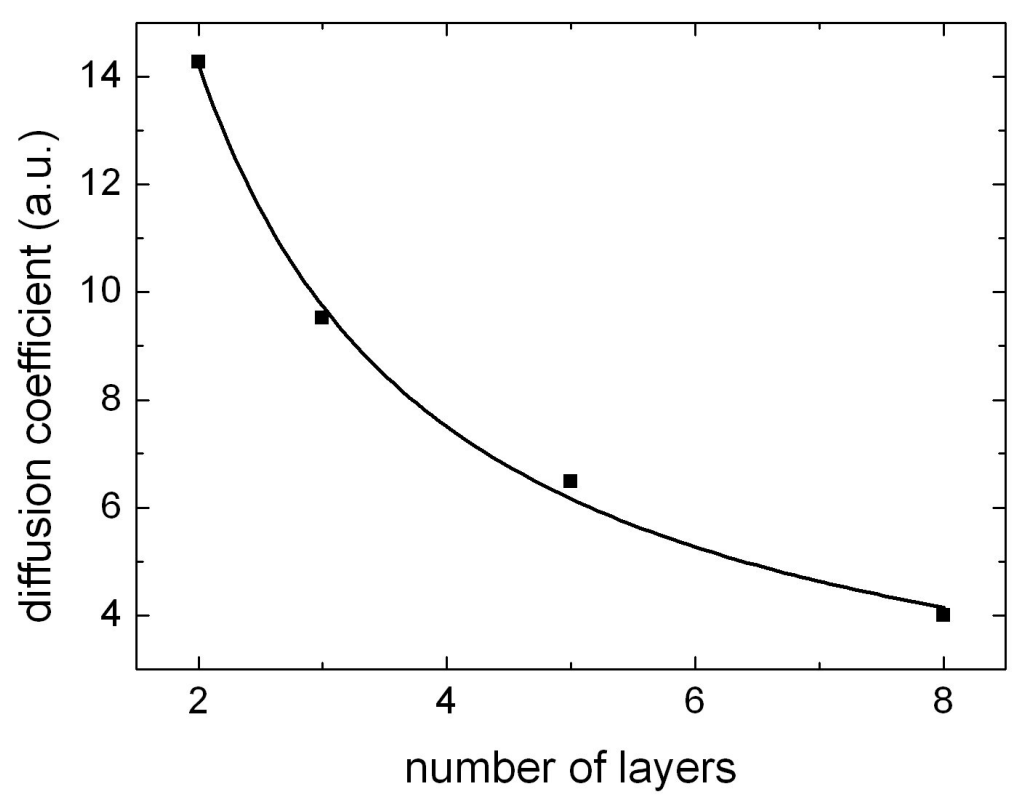

Figure 5.10: Simulated values for the film thickness dependence of the diffusion coefficients using a Gay-Berne-Kihara model. The simulations were carried out by Marco G. Mazza.

\subsection{Diffusion in proximity to phase transitions}

After finding some particularities for the behaviour of the diffusion in molecularly thin freestanding film, the properties of the diffusion around phase transitions is an obvious candidate to study. First of all it is of interest if there is a difference in the scaling depending on the film thickness. As the thinner films show a surprisingly fast diffusion, one might expect that it could scale differently with temperature. Secondly, there is a special interest in those liquid crystals that show a transition to the hexatic or the crystalline smectic phase. Here, a drastic change in the diffusion coefficient is expected due to the higher order of one of the phases. As the phase transition occurs layer by layer, the dye molecules can probe the behaviour at a molecular scale in such inhomogeneous films. The possibility to study the diffusion in axially heterogeneous films will be approached in a separate section.

\subsubsection{Temperature dependence within the smectic A phase of $8 \mathrm{CB}$}

The first mesogen that is studied is again $8 \mathrm{CB}$. This liquid crystal is in the smectic A phase at room temperature in the temperature range between $22^{\circ} \mathrm{C}$ and $33.8^{\circ} \mathrm{C}$. In bulk, $8 \mathrm{CB}$ exhibits 
a transition to the nematic phase at higher temperatures and to the crystalline phase at lower temperature. However, the transition to the crystalline phase is more complicated. The values from table 4.1 are the values obtained by calorimetric measurements upon heating the sample. It is frequently observed that $8 \mathrm{CB}$ can easily be undercooled into the bulk crystalline region for long times without any sign of a phase transition. Sometimes this is attributed to geometrical confinement [119]. However, also for bulk 8CB calorimetry shows no transition to the crystalline state at all when the temperature cycles do not reach temperatures that are low enough [120]. The transition upon cooling turns out to be around $-5^{\circ} \mathrm{C}$ [121]. The observation for the free-standing films prepared at room temperature is that the films can easily be undercooled far below the bulk phase transition temperature down to $0^{\circ} \mathrm{C}$; no transition to the solid phase were found even after equilibration times of more than a day. For thin free-standing films, the transition to the solid phase causes the rupture of the film as the external stresses cannot be compensated by the elasticity of the film anymore.

For temperatures higher than the bulk smectic range, it was found for thick films of $8 \mathrm{CB}$ that they show a subsequent thinning upon heating [42]. This reduces the film thickness down to 10 layers for temperatures close to the bulk transition temperature to the isotropic phase. This in turn means that films thinner than ten layers are stable in the complete nematic range and can be studied in the whole bulk nematic temperature range. Sometimes the transition region between nematic and smectic phase is connected to a reduced diffusion coefficient [122] which, however, is not found consistently [123]. Regardless of the bulk phase for the measured temperature the film has to be in the smectic state to exist as a free-standing film.

As the diffusion differs between films with different film thicknesses - as it was shown in the previous section - one can measure the temperature dependence for all possible numbers of layers. As the first example, a film with seven smectic layers will be considered. As mentioned, the film could be undercooled into the bulk solid state so that the dependence of the diffusion coefficient on the temperature $T$ could be measured in a range between $0{ }^{\circ} \mathrm{C}$ and $39^{\circ} \mathrm{C}$ as shown in figure 5.11. It is found that the data can be fitted very well using the classical Arrhenius equation

$$
D=D_{0} \exp \left(-\frac{E_{a}}{R T}\right)
$$

with the activation energy $E_{\mathrm{a}}$, the gas constant $R$, and the limit $D_{0}$ of the function for $T \rightarrow$ $\infty$. For temperatures lower than $0^{\circ} \mathrm{C}$ the film ruptures. For a free standing film this can be indicative of a transition to the crystalline state. This observation of the transition to the 

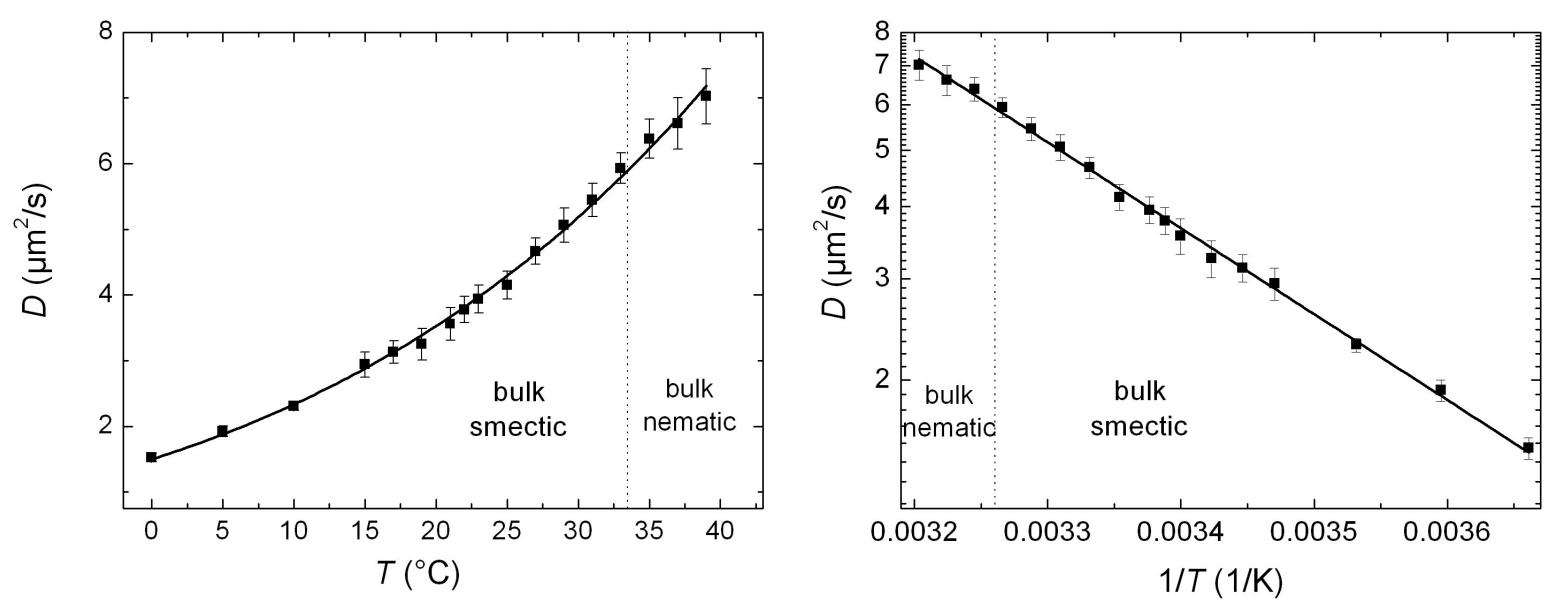

Figure 5.11: Temperature dependence of diffusion for a seven layer film (left) and as Arrhenius plot (right) with logarithmic scale of $D$ over $1 / T$. The bulk phase behaviour is indicated with suppressed crystalline phase. The solid lines show the fit using equation (5.12).

crystalline phase at around $-5^{\circ} \mathrm{C}$ coincides well with the mentioned calorimetric observations for bulk 8CB upon cooling [121].

To get full information about the diffusion for all free-standing films, for all possible film thicknesses the temperature dependences would have to be measured. However, mainly for the thinnest films changes in the behaviour would be expected. It turns out that the films consisting of only two smectic layers are not stable anymore at elevated temperatures higher than $31^{\circ} \mathrm{C}$. For the other film with a thickness between 3 and 7 layers the temperature dependence was measured in the range of the smectic and the nematic phase. Lower temperatures were not measured in order to avoid discussions about possible solid clusters in the undercooled smectic state.

The Arrhenius plots for the mentioned film thicknesses are shown in figure 5.12 together with the fits using equation (5.12). The decay constant $E_{\mathrm{a}} / R$ of the Arrhenius function is the characteristic value for the temperature dependence of the diffusion coefficient. The overview over the fitted decay constants for the measured films are shown in table 5.2. The obtained activation energies are close to the values reported for nematic $8 \mathrm{CB}$, which are around $E_{\mathrm{a}} / R=$ $3800 K$ [124].

The temperature dependence of the films appears to be the same for all the different thicknesses within $\pm 5 \%$ of the average. This variation is below our experimental precision given the visible scatter of the data in figure 5.12. Thus, it can be assumed that the scaling with 


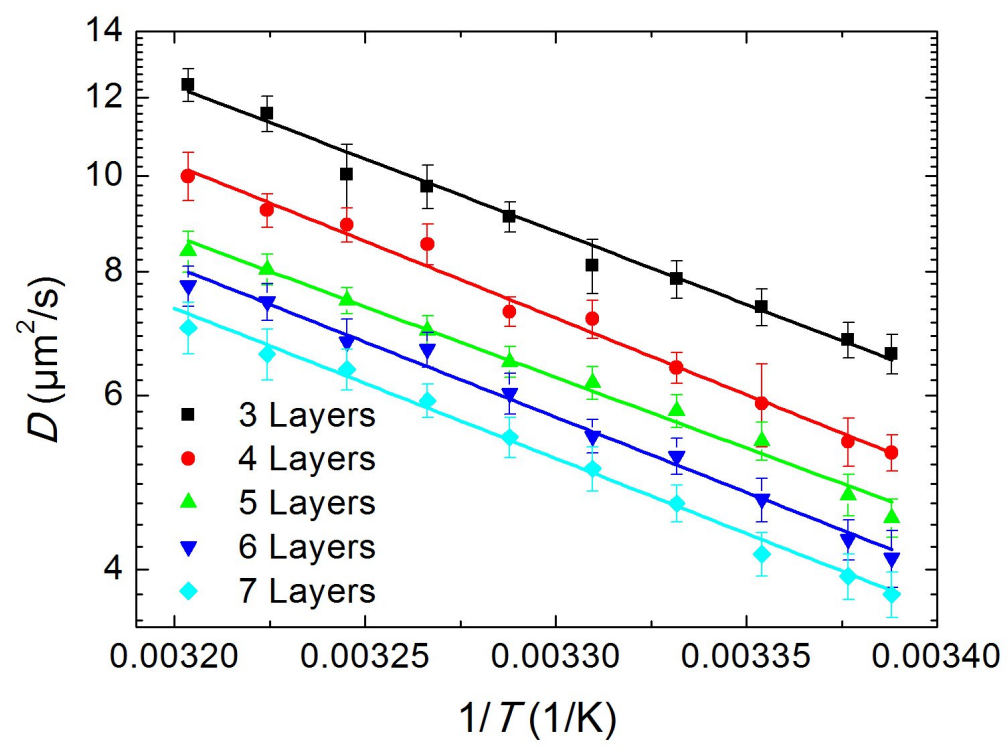

Figure 5.12: Arrhenius plots for three to seven layers of 8CB. The fits are done using (equation 5.12 .

\begin{tabular}{|c|c|}
\hline number of layers & $E_{\mathrm{a}} / R(\mathrm{~K})$ \\
\hline \hline 3 & 3380 \\
\hline 4 & 3560 \\
\hline 5 & 3280 \\
\hline 6 & 3500 \\
\hline 7 & 3490 \\
\hline
\end{tabular}

Table 5.2: Decay constants of the Arrhenius fits shown in figure 5.12 for three to seven layers of $8 \mathrm{CB}$.

temperature is not dependent on the film thickness. This means that we know the change of the diffusion coefficient with the film thickness from the previous section and we know the scaling with temperature. Hence, together with the results obtained in section 5.1.1, one can write the general empiric equation for the diffusion coefficient of free-standing $8 \mathrm{CB}$ films of $n$ layers thickness at a temperature $T$ as:

$$
D=2.12\left(1+\frac{7.14}{n}\right) \exp \left(-\frac{3440 \mathrm{~K}}{T}\right) \frac{\mu \mathrm{m}^{2}}{s} .
$$




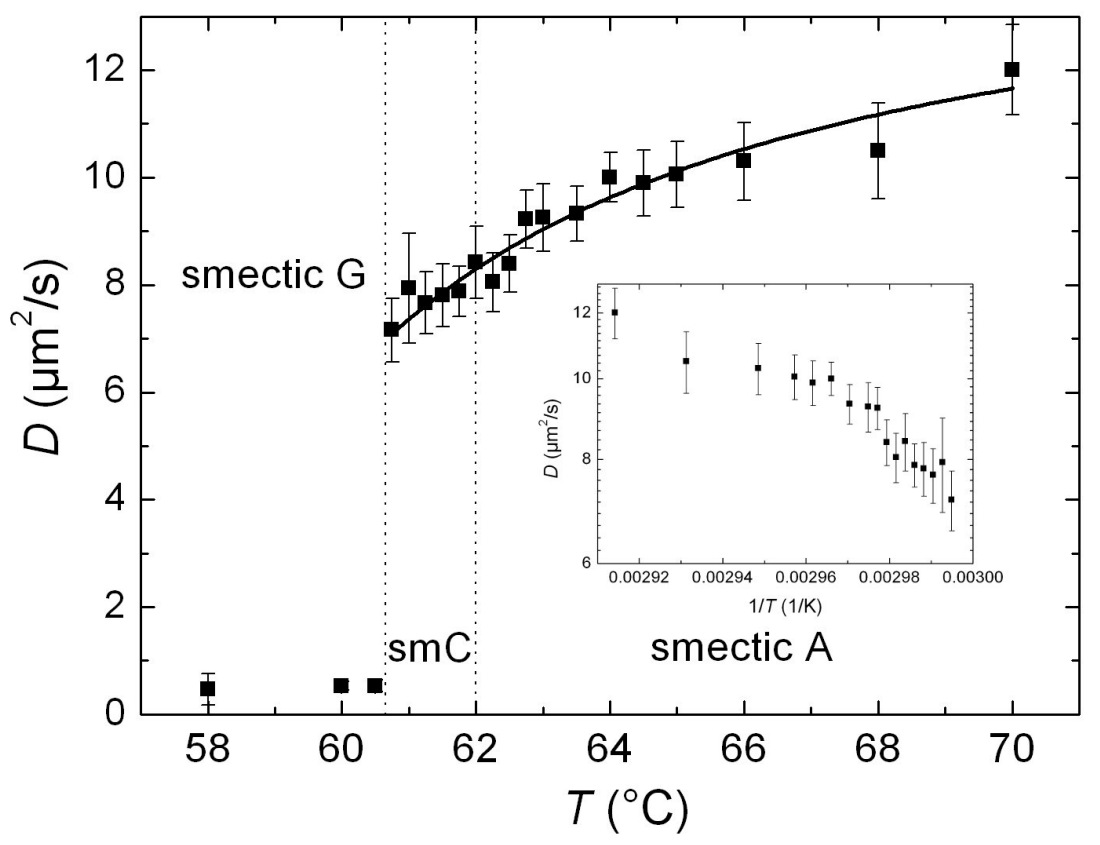

Figure 5.13: Temperature dependence of the diffusion coefficient of a 10 layer film of 70.4. The solid line shows a fit using equation 3.8. The inset is an Arrhenius plot of the measured values.

\subsubsection{Diffusion close to transitions with in-plane ordering of 70.4 and 90.4}

The temperature dependence of the diffusion coefficient observed for $8 \mathrm{CB}$ shall be compared to the ones of other liquid crystal materials. Firstly, pyrimidine was considered with its transition from the smectic $\mathrm{C}$ to the crystalline phase. However, this material does not seem to form stable free-standing films in the smectic $\mathrm{C}$ phase. The preparation of stable free-standing films in the smectic A phase is possible but as soon as the film enters the smectic $\mathrm{C}$ phase, material starts flowing into the central region of the film and forms a thick equilibrium film of several microns thickness. Hence, pyrimidine could not be studied with respect to diffusion when approaching a higher ordered phase.

The next candidate is 70.4 with a transition from the smectic A to the smectic $\mathrm{C}$ phase and from the smectic $\mathrm{C}$ to the smectic $\mathrm{G}$ phase. This transition has the advantage that out of the different considered states the highest ordered one is still smectic so that the film remains stable without disruption. One can thus measure both sides of the phase transition. As shown in figure 5.13 the behaviour is different from the case of $8 \mathrm{CB}$. The first observation for this 

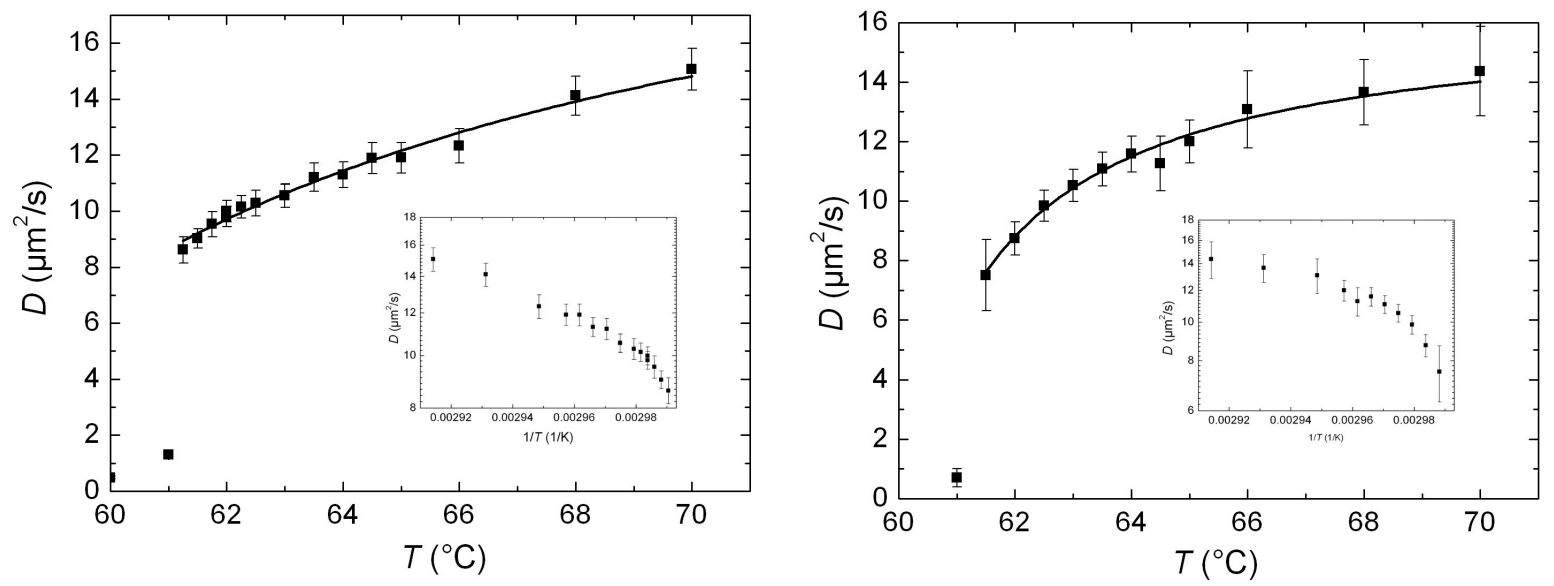

Figure 5.14: Temperature dependence of the diffusion coefficient of 70.4 for a film thickness of 3 (left) and 6 (right) layers. The insets show that the Arrhenius plots do not give a straight line.

example of a film consisting of 10 layers is the absence of any jump in the phase transition from the smectic A to the smectic $\mathrm{C}$ phase. This is expected as it is a second order transition. Upon further cooling below the transition temperature to the smectic $\mathrm{G}$ phase, the diffusion coefficient drops drastically by around one order of magnitude. For the functional dependence on the temperature it is not possible to fit the data with the simple Arrhenius function (equation (5.12) ) contrary to the case of $8 \mathrm{CB}$. One can, however, use the function for the case of a glass transition (equation (3.8)). This property is also confirmed for the film thicknesses of three and six smectic layers as depicted in figure 5.14. This means that the transition to a higher ordered smectic phase can be described with the equations of a glass transition whereas the smectic-crystalline transition of $8 \mathrm{CB}$ did not show any sign of a closeby phase transition but followed the classical Arrhenius behaviour in the entire observed temperature range.

The diffusion coefficient also changes gradually for the case of a film with three layers. This shows that the observed slowdown upon cooling the sample is not caused by a subsequent freezing of the layers in the sample. This might lead to a similar effect for a sufficiently thick film when assuming that the tracked molecules always average over all the existing layers. In fact the steady change of the diffusion coefficient for the case of only three layers shows that the diffusion has to change in all the layers when approaching the phase transition. As we will see in the following section, one can recognise if there is an inhomogeneous diffusive behaviour with the help of the used video microscopy. 
It was shown that the phase transition in some of the free-standing films appears to scale like a glass transition. It would therefore stand to reason to also compare further observations made in free-standing polymer films to the ones in our smectic free-standing films. The effect most studied in thin polymer films is the change in the glass transition temperature $T_{\mathrm{g}}$. For thicknesses smaller than roughly $30 \mathrm{~nm}$ a decrease of the phase transition temperature is measured. The empirical scaling law is [125]

$$
T_{\mathrm{g}}=T_{\mathrm{g}}^{\mathrm{bulk}}\left[1+\left(\frac{a}{h}\right)^{\delta}\right],
$$

with the bulk glass transition temperature $T_{\mathrm{g}}^{\mathrm{bulk}}$ and the thickness $h$. The values of the fit parameters for the example of polystyrene are $a=3.2 \mathrm{~nm}$ and $\delta=1.8$ [125]. Applying this equation to a film of three layers thickness would lead to a change in the transition temperature of more than $50 \mathrm{~K}$. Contrary to that, no measurable change in the 70.4 films could be found. Also for the diffusion in free-standing polystyrene films no changes were found when varying the film thickness for values bigger than $30 \mathrm{~nm}$ [126]. Thus, the scaling with temperature is similar between smectic and polymer free-standing films whereas the other physical properties do not seem to agree. This, however, is not surprising as the glass transition is not a thermodynamic phase transition contrary to the transition to the smectic crystalline phase.

For 7O.4, strong fluctuations of the diffusion coefficient by more than $20 \%$ were predicted from indirect measurements using general relations between the thermal conductivity and heat capacity [127]. None of our measurements showed any sign of this behaviour although the resolution would be good enough to detect such strong variations. Possibly the indirect measurement fails for the fluctuations close to phase transitions, but more probably the fluctuations get suppressed in a free-standing film.

In order to get more insight into the different properties of the diffusion of $8 \mathrm{CB}$ and 70.4 , one can also analyse the diffusion data of $8 \mathrm{CB}$ in a different way. The idea is that the phase transition of $8 \mathrm{CB}$ from the crystalline to the smectic A state might get suppressed in confined geometries [119]. However, the phase transition would be visible in the extrapolated bulk diffusion values $D_{\infty}$ from equation (5.4). Since the temperature dependence is known for the films consisting of 2 to 7 smectic layers, one can vice versa analyse the thickness dependence of the diffusion for the different temperatures in the same way as it was done in section 5.1 .1 for the case of room temperature. From this, one obtains the $D_{\infty}$ for the temperature range between $22^{\circ} \mathrm{C}$ and $39^{\circ} \mathrm{C}$. The dependence is shown in figure 5.15 together with the Arrhenius 


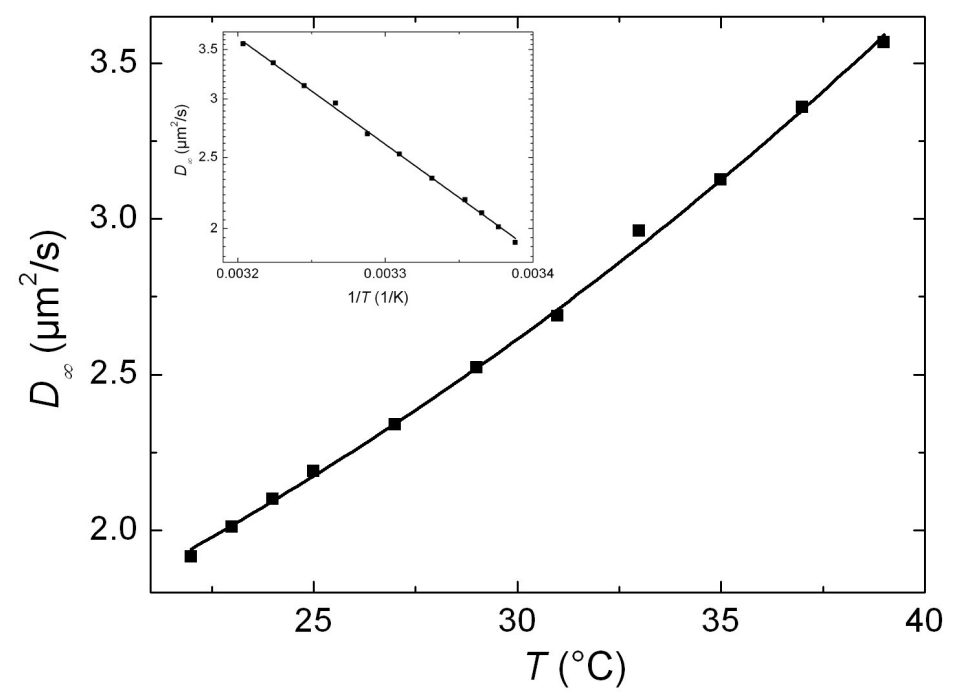

Figure 5.15: Extrapolated bulk values $D_{\infty}$ of the diffusion in $8 \mathrm{CB}$. The solid line is a fit using equation (5.12). The inset shows the Arrhenius plot of the data.

plot of the data set. For the calculation of the $D_{\infty}$, the parameter $A$ from equation (5.4) was set to the constant value of 7.1, obtained in section 5.1.1, in order to have $D_{\infty}$ as the sole fitting parameter. The solid line in the figure is a fit for the classical Arrhenius law. No deviation from the solid line is found which would indicate the proximity of the phase transition to the crystalline phase. Also the decay constant of the shown Arrhenius fit $E_{\mathrm{a}} / R=3330 \mathrm{~K}$ falls between the values obtained for the thin films that were presented in table 5.2 .

However, this method of extrapolating the data obtained for only six different film thicknesses does not appear to be the most reliable method of calculation. Still the results agree very well with the observations made for the thin films. One can therefore conclude that the temperature dependence of the diffusion of $8 \mathrm{CB}$ and 70.4 appears to be fundamentally different.

A similar behaviour as for 70.4 is expected for the molecule 90.4 as it has the same functional group just with a longer aliphatic tail. The phase transition of interest in this case is the transition from the smectic A to the hexatic smectic F state. The observed behaviour is in fact very similar to the one of 70.4, with a functional dependence of the diffusion on temperature like for a glass transition. However, the material turns out to be unstable in air, so that the phase transition temperature changes with time. For a film consisting of ten smectic layers the diffusion coefficients measured over 15 days are shown in figure 5.16. For every 


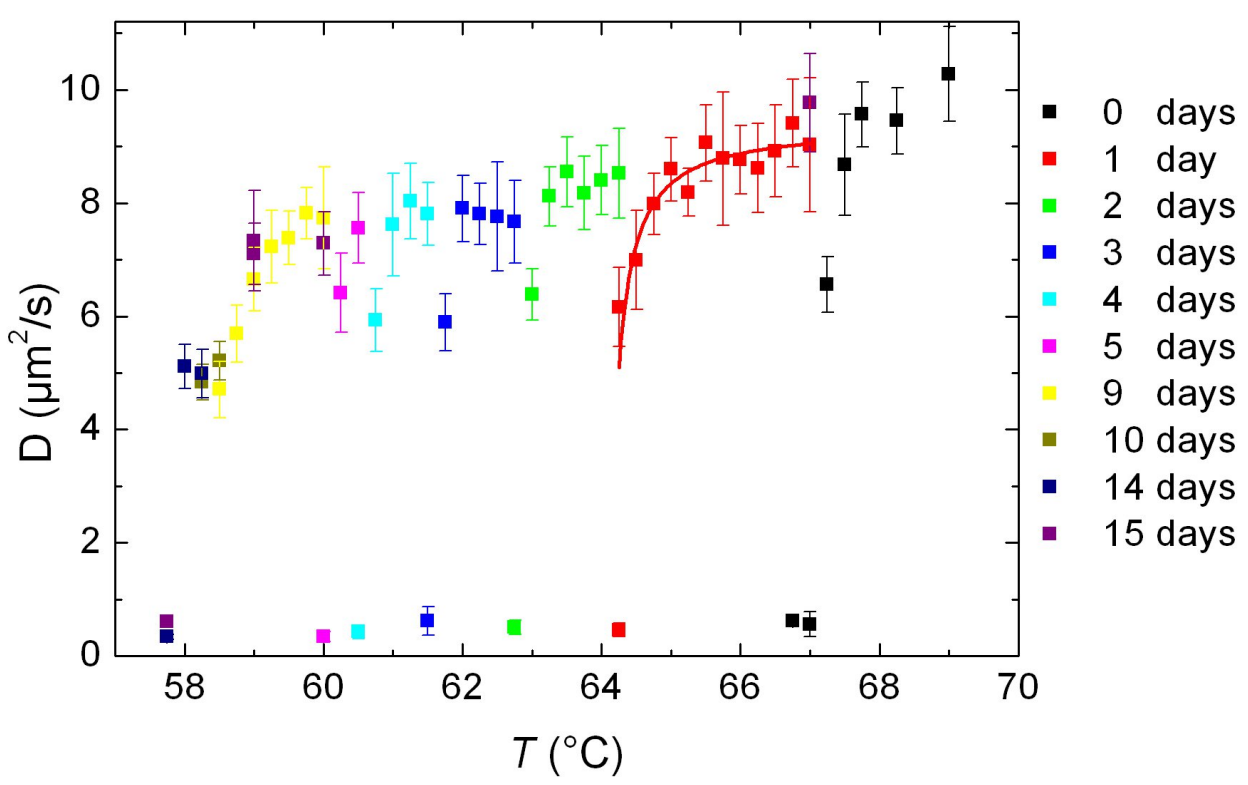

Figure 5.16: Temperature dependence of the diffusion coefficient in 90.4 measured over 15 days. The solid line shows the fit using equation $(3.8)$ for the first day as this series provides the biggest data base.

series of measurement the diffusion was recorded upon reducing the temperature until a visible slowdown of the diffusion was observed, displaying the transition of the film into the hexatic phase. The faster diffusion for a later time showed the recovery of the smectic A phase. The visible changes in the films over time make it difficult to find a general conclusion about the diffusion in the film. But as the changes appear to be slow and on the time scale of days, one can analyse the data set of one day (which still means acquired over a time span of around 10 hours). This was done for the example of the measurements after one day (red squares). As can be seen by the fit (red solid line), the behaviour close to the phase transition is again according to equation (3.8), which means that the diffusion coefficient close to the transition to the hexatic phase can be described with the temperature dependence of a glass transition.

However, if one only considers a selection of data points which are not directly at the phase transition, one finds that the temperature dependence can be described with the normal Arrhenius equation. This is shown in figure 5.17, Despite the large error bars present for those measurements, one finds a general agreement and no deviation from the classical Arrhenius behaviour as it is evident in figure 5.13 or 5.14. It is found that already at a temperature difference of two Kelvin from the phase transition temperature, one cannot decide anymore what 


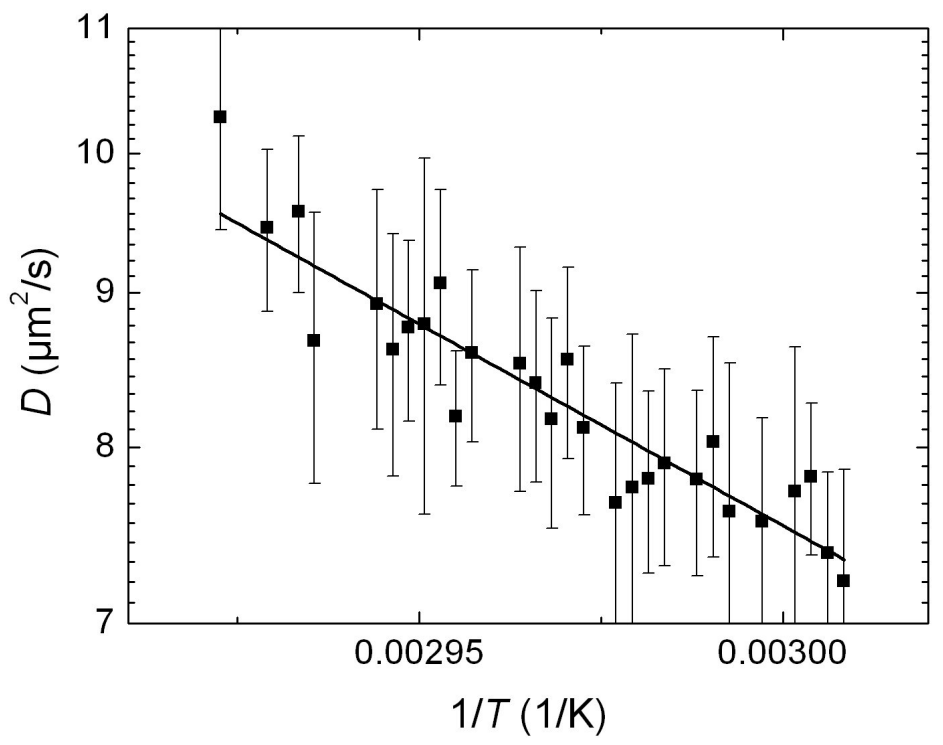

Figure 5.17: Arrhenius plot for the diffusion of 90.4 using only data points with at least $1.5 \mathrm{~K}$ temperature difference from the phase transition. The data is the same as in figure 5.16 neglecting the direct vicinity of the phase transition

kind of a scaling is present. The reason - apart from the limited precision of the measurement method - is that the studied temperature range (and the induced changes in diffusion coefficient) is too small so that the temperature dependence is nearly linear.

One can also analyse the change in the phase transition temperature with time for the same film of 90.4. Using the temperature where the drop in the diffusion coefficient is found in figure 5.16 one obtains the changing phase transition temperature depicted in figure 5.18 . The solid line in the figure is an exponential fit of the data, which indicates that by the end of the measurements an equilibrium is reached. Obviously the 90.4 molecules are not stable in air. An instability might be caused either by an imine-enamine tautomerism [128] or most probably by hydrogenation of the double bond between carbon and nitrogen. However the structural changes do not seem to affect the diffusive behaviour too much (unless directly close to the phase transition) as the diffusion measurements at higher temperatures after 15 days agree to the ones taken before (see the purple data points of figure 5.16). 


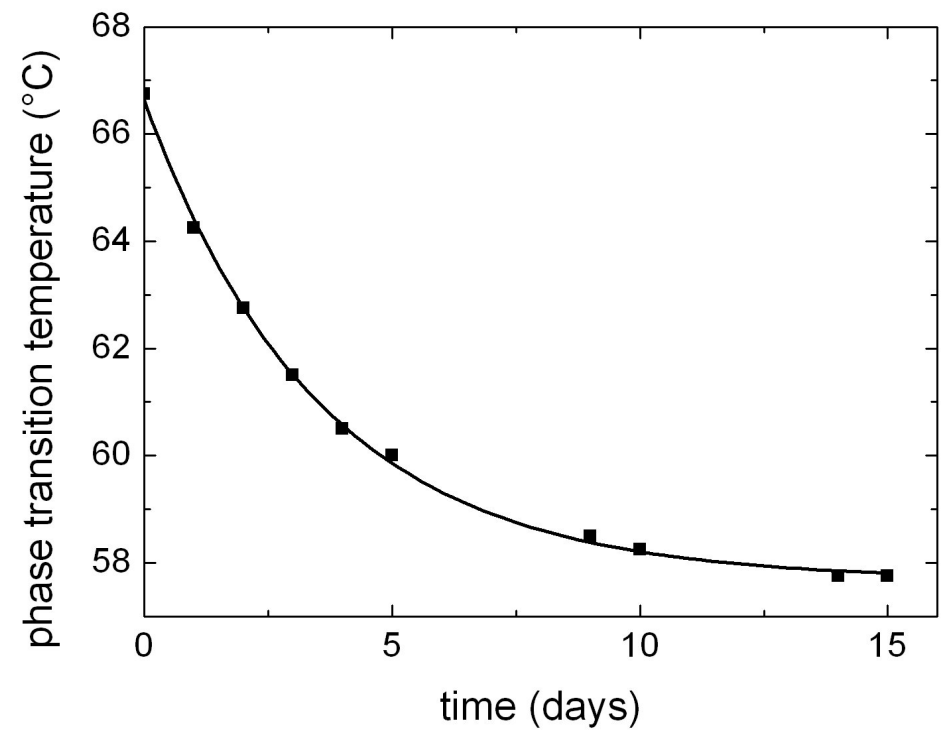

Figure 5.18: Change of the phase transition temperature of 90.4 with time. The considered transition is from the smectic A to the smectic F state. The solid line shows an exponential fit to the data.

\subsubsection{First order transition from smectic A to smectic C of C7}

The last mesogen that shall be considered here is C7. It has a phase transition to the smectic $\mathrm{G}$ phase, that might be studied. However, in our experiments the films were invariably breaking for temperatures below $49^{\circ} \mathrm{C}$. Thus, the transition to the smectic crystalline phase could not be studied. Because of this, the focus was on the transition from the smectic A to the smectic $\mathrm{C}$ phase. This transition is usually of second order and would not lead to visible effects at the phase transition. This kind of behaviour was observed for the case of 70.4 as it was shown in the figures 5.13 and 5.14. However, for the compound C7 this transition is first order [83]. The situation is slightly different for free-standing films where the first order character is suppressed in very thin films [87, 108]. For this reason, no effect would be expected for the case of three smectic layers. Figure 5.19 compares the diffusion coefficient in vicinity of the phase transition for a film consisting of three layers and one of seven layers. For both of the cases there is a clear dip around the phase transition. Apparently, despite the change of the character of the phase transition, the properties of the diffusion remain unchanged. A similar behaviour of a reduced diffusion coefficient was also observed for continuous transitions in gels, where it was explained by internal density fluctuations at the phase transition temperature [129]. As the mechanism that suppresses the first order character is not known, the fluctuations 


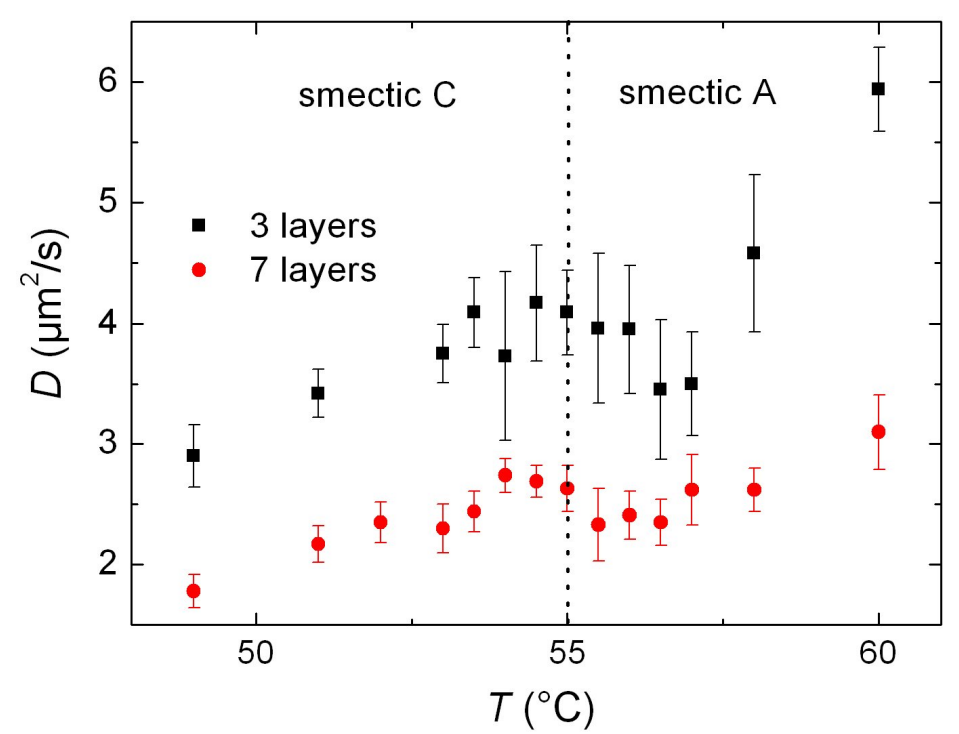

Figure 5.19: Diffusion coefficients around the phase transition from smectic C to smectic A for $\mathrm{C}$. The dotted line only gives the approximate phase transition temperature.

that cause the smectic $\mathrm{C}$-smectic A transition to be first order [15] might still be present and lead to a slower diffusion also for a small number of layers.

As conclusion of this section, it was shown that the fundamental nature of the diffusion does not vary with the number of layers in a free-standing film for the same material. The unexpectedly high values of the diffusion coefficient for thin films presented in the previous section gave rise to the expectation of changes in the scaling with temperature. This, however, could not be observed for any of the considered materials. Even the change in the order of the phase transition for the molecule $\mathrm{C} 7$ does not seem to affect the main characteristics.

Nevertheless significant changes depending on the type of phase transition could be found. The transition to a hexatic or a crystalline smectic phase can be described like a glass transition. Contrary to that, the proximity of the phase transition to the crystalline phase was not found to have a representation in the diffusion coefficient and showed the classical Arrhenius behaviour. 


\subsection{Introduction of zero-shear viscosity in free-standing films}

One can also use the data presented in the first two sections of this chapter to calculate the viscosity $\eta$ in our free-standing films. Usually there are more precise ways to measure the viscosity than by the analysis of the diffusion of tracer particles. However, smectic liquid crystals show a strongly non-Newtonian behaviour [130]. This means that the classical definition of the dynamic viscosity as being the proportionality constant between the shear rate $\partial u / \partial y$ (with the shear velocity $u$ and the $y$ dimension perpendicular to the shearing) and the shear stress $\tau$ cannot be applied as the two quantities are not proportional anymore. This means that only a shear rate dependent value called as apparent viscosity can be defined:

$$
\eta=\frac{\tau}{\partial u / \partial y}
$$

For example, for $8 \mathrm{CB}$ the apparent viscosity is observed to change by around 3 orders of magnitude when the shear rate is varied by four orders of magnitude [131]. Also, for very low shear rates down to $\tau=0.01 \mathrm{~s}^{-1}$ the value of the apparent shear viscosity does not converge to a constant value. Our measurement method has the advantage that is can measure the viscosity for the case of zero shear. The calculation is simply done via the Stokes-Einstein equation (3.7). The radius was estimated as half of the long axis of the Nile red molecule, $a=0.7 \mathrm{~nm}$. One can now plot for example the temperature dependence of the zero-shear viscosity of the free standing $8 \mathrm{CB}$ film consisting of seven layers, as shown in figure 5.20 . Because of the inverse proportionality between diffusion coefficient and viscosity, the data can be fitted by an exponential function again. The absolute values of the observed viscosities are very close to the ones found for the apparent viscosity in free-standing films [111]. The values also differ only by a factor of two from the values found for $8 \mathrm{CB}$ in the nematic phase for bulk shear experiments [130]. This means that the values of the order of several hundred $\mathrm{Pa} \cdot \mathrm{s}$ observed for small shear rates in the smectic phase of 8CB [131] appear to be caused more by the rigidity of domain boundaries than corresponding to the properties at molecular scale.

This presented temperature dependence, however, is just one example for the information about the introduced zero-shear viscosity that we can give now. Because of the close relation to the diffusion coefficient, all the statements made on the behaviour of the diffusion close to phase transitions also apply to the behaviour of the viscosity accordingly. Thus, it is possible 


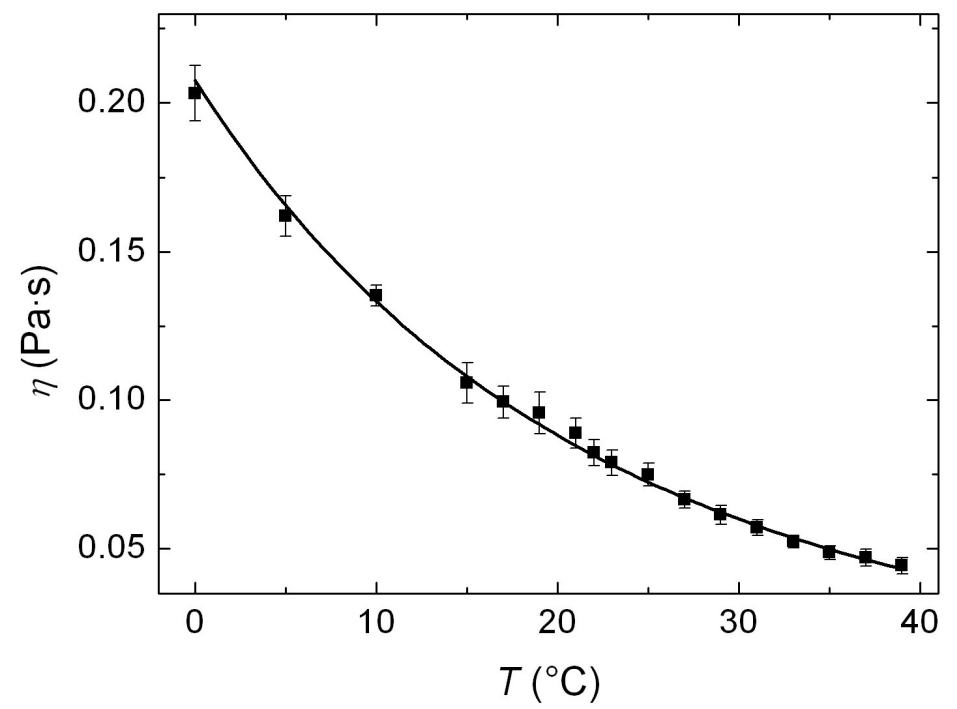

Figure 5.20: Temperature-dependence of the zero-shear viscosity in an 8CB film consisting of seven layers. The solid line is an Arrhenius fit to the data.

to write the expression for the viscosity for any possible film thickness and temperature in the same way as for the diffusion coefficient in equation (5.13).

The viscosity in liquid crystal is inherently anisotropic because of the anisotropic shape of the molecules [132]. With the presented experiments only one viscosity coefficient is possible to be accessed. To name this value, it is not possible to refer to the notation of Helfrich [133] as this is defined in reference to the shear direction that does not exist in our way of determining the viscosity. This indirect definition with the help of the diffusion coefficient leads to only two distinct viscosities, according to the two existing diffusion coefficients in liquid crystals. The viscosity measured here is the one perpendicular to the director; it should therefore be called $\eta_{\perp}$. The other viscosity coefficient $\eta_{\|}$, however, is not accessible with the applied method.

\subsection{Diffusion in heterogeneous free-standing films}

In this section, the properties of heterogeneous free-standing films will be analysed. Inhomogeneities are common observations in free-standing films close to phase transitions. Here, the analysis will be restricted to the study of transitions from the smectic A/C to the hexatic/crystalline smectic phases as the transition smectic $\mathrm{A}$ to smectic $\mathrm{C}$ cannot be identified 

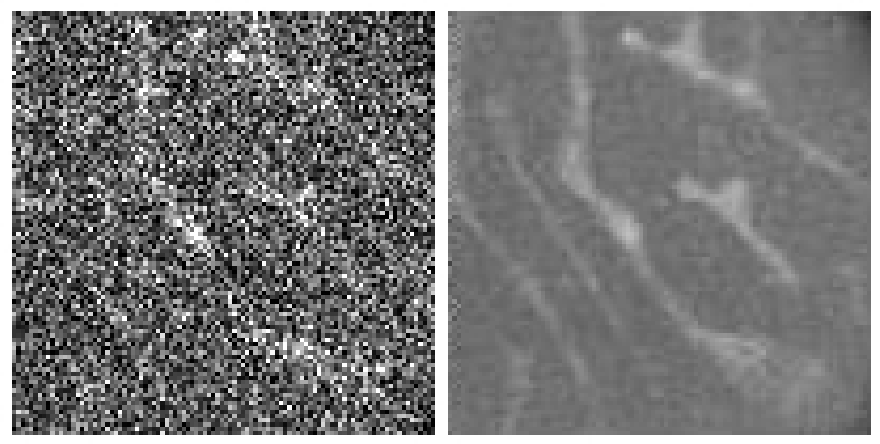

Figure 5.21: Left: Real image of a laterally inhomogeneous film in the fluorescence microscope showing the brighter regions only slightly due to the dominating dark noise; Right: The same film averaged over 45 frames. The regions with the brighter background are in the less ordered state (smectic $\mathrm{A}$ in this example) and darker regions in the higher ordered state (smectic $\mathrm{F}$ here). The dimensions are $45 \times 45 \mu \mathrm{m}^{2}$ for both images.

with sufficient precision. The two cases of axially and laterally inhomogeneous films will be discussed separetely.

\subsubsection{Laterally inhomogeneous films}

The laterally inhomogeneous films are invariably inequilibrium films and equilibrate to homogeneous films in the time scale of minutes to hours. The identification of the heterogeneity can simply be done with the help of the fluorescence microscope as regions with different phases display a different background signal as shown in figure 5.21. The real image on the left side only faintly gives an idea of the different background intensities but averaging over 45 frames on the right side reveals the structures defining different regions of the frame. As diffusing molecules are visibly faster in the brighter regions, those regions can be identified to belong to the less ordered state. The possible explanation for higher background in the more mobile regions is that the patches with material in the smectic A state gets surrounded by hexatic material [134] resulting in a larger film thickness of the regions with smectic A material (illustrated in figure 5.22). This larger film thickness causes a larger background signal in these regions. The inequilibrium property of the film is also manifested by the convection that the described patches always exhibit.

We will now consider a free-standing film of 90.4 consisting of ten layers with its transition from the smectic $\mathrm{F}$ to the smectic A phase. The general observation for these films is 


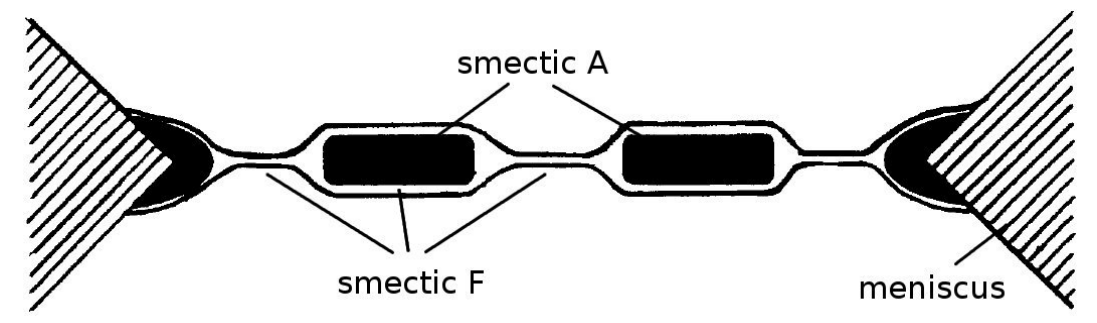

Figure 5.22: Schematic cross section of a laterally inhomogeneous film for the example of 90.4: The regions with smectic A material are sourrounded by material in the smectic F state. Image adapted from [134].

that the dye molecules rarely leave the respective region they are in. This means that the fast diffusing molecules in the smectic A state stay within the thin region shown in figure 5.21 with size of only around two microns in width. This can be visualized by overlaying an averaged brightness image with the found trajectory of the molecules as shown in figure 5.23. Contrary to the previous figure, in this case the averaging was done over 150 frames (corresponding to 3 seconds). During this time the smectic A patches are moving so that the bright regions smear out a bit and the contrast becomes smaller. This fluorescence image is overlaid with two trajectories identified during this time. One finds that the trajectories always stay in the brighter regions.

Apart from this visual observation of the molecules staying in one of the two phases one can also analyse the diffusion coefficients for this purpose, in this case ignoring the drift of the molecules. If the molecules changed their surrounding phase regularly, the diffusion coefficients would average over the short step length of the hexatic phase and the longer step length of the smectic A phase. The analysis of the single steps, in contrast, would show a separation of the shorter and larger steps due to the spatial separation of the two phases. Both, the distribution of the diffusion coefficients and the occurrence of the single steps are shown in figure 5.24. The inset in the distribution coefficient shows the corresponding distribution for a film at $3 \mathrm{~K}$ above the phase transition. No broadening of the distribution is found. For both curves the diffusion coefficient can be determined assuming independent diffusion in distinct regions. In case of the distribution the averages are obtained from the center of a fit using two Gaussians. For the step length probabilities the diffusion coefficient can be calculated by the decay lengths of a double exponential fit using equation (3.5). The calculated values for the slow diffusion $D_{\text {hex }}$ and the fast diffusion $D_{\text {smA }}$ for both methods are shown in table 5.3. Of 


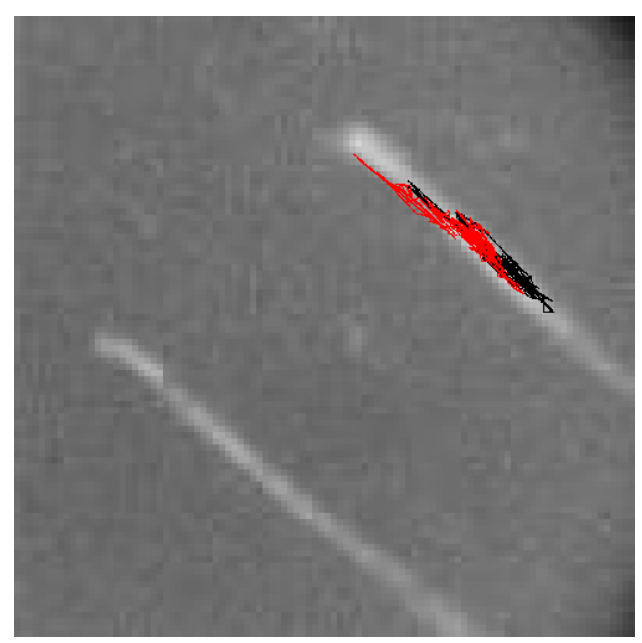

Figure 5.23: Image of the fluorescence microscope averaged over 150 frames overlaid by trajectories of two molecules (shown in black and red) diffusing only within the brighter smectic A phase. The image dimensions are $45 \times 45 \mu \mathrm{m}^{2}$.

\begin{tabular}{|c|c|c|}
\hline method & $D_{\text {hex }}\left(\mu \mathrm{m}^{2} / \mathrm{s}\right)$ & $D_{\text {smA }}\left(\mu \mathrm{m}^{2} / \mathrm{s}\right)$ \\
\hline \hline distribution & 0.8 & 6.2 \\
\hline step lengths & 1.1 & 5.4 \\
\hline
\end{tabular}

Table 5.3: Diffusion coefficients $D_{\text {hex }}$ and $D_{\text {smA }}$ for a laterally inhomogeneous film calculated either by the distribution of diffusion coefficients or by the probability of single step lengths.

course particularly because of the few slow reconstructed trajectories the value for the slow diffusion coefficient is not very reliable. However, one finds a general agreement between the two methods indicating that there is no major interconnection between the two phases and crossing of phase boundaries appears to be a rare event.

\subsubsection{Axially inhomogeneous films}

The axially inhomogeneous films are to some extent more interesting as it is a particular case of the hexatic phases that one can observe a layer by layer phase transition in the freestanding films [17]. This means that slightly above the bulk phase transition temperature the layers closer to the surface show phase transitions at higher temperatures than the layers in the middle of the film. For our tracking experiments, this could result in the possibilty to identify 

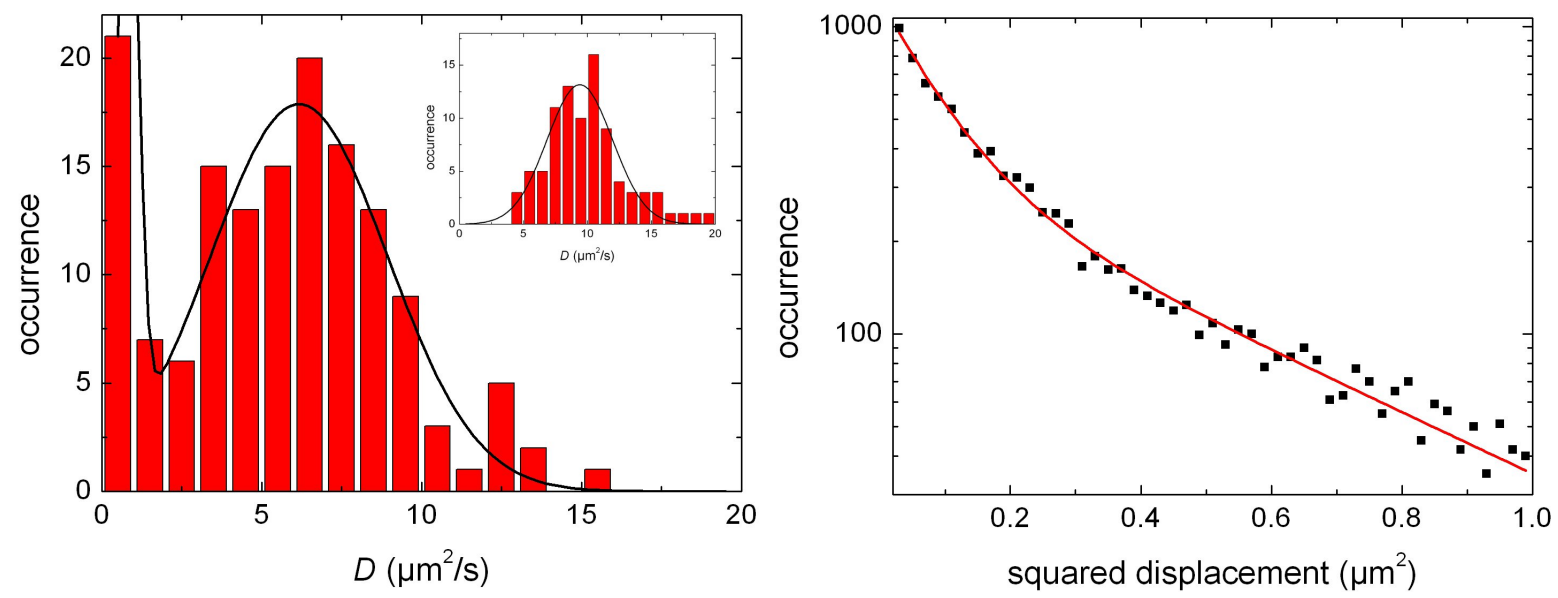

Figure 5.24: Diffusion in a laterally inhomogeneous film. Left: Distribution of diffusion coefficients with a fit for two Gaussian distributions. The inset shows the distribution for a film at a temperature $3 \mathrm{~K}$ above phase transition for comparison of the distribution width. Right: Probabilities for the step length with a double exponential fit.

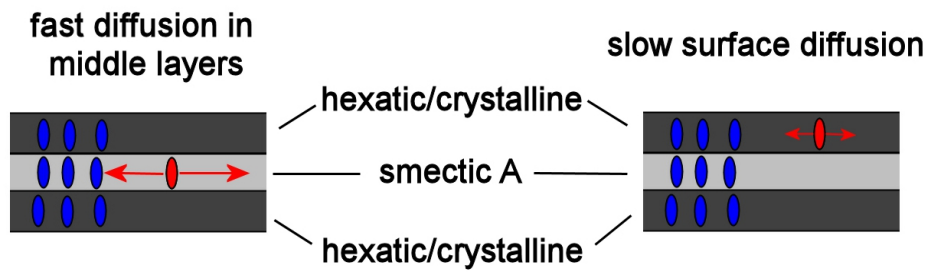

Figure 5.25: Layer dependent diffusion in a film close to a hexatic phase transition. The molecules in the center of the film are still in the smectic A phase and show fast diffusion while molecules in the surface layers show the slow diffusion of the hexatic phase.

the location of a molecule in a film purely because of their diffusive behaviour. The condition for this would be that the observed dye molecules would have to stay long enough in one phase. This is visualised in figure 5.25. In the surface layers the diffusion would be slowed down significantly while in the middle of the film the molecules would still show the faster diffusion of the smectic A phase.

We now consider a free-standing film of 90.4 consisting of seven smectic layers. The analysed trajectories describe molecules that were tracked over a time of at least 1 second. In this time the molecules travelled a distance of more than 5 microns when in the smectic A phase. The thin sheet in the smectic A phase, however, only has a thickness of a few 


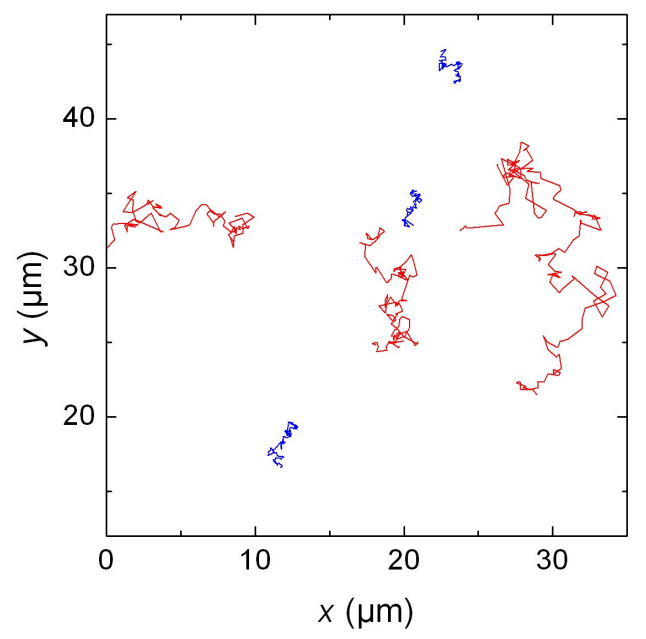

Figure 5.26: Three examples for both slow (blue) and fast (red) molecules in an equilibrium film of 90.4 cosisting of 7 layers.

nanometers. This means that if it is possible to find molecules that stay in the middle layer this will be a strong indication that molecules avoid crossing phase boundaries. The experimental observation is that it actually is possible to find both fast and slowly diffusing molecules in an equilibrium film. Examples for trajectories reconstructed for one film are shown in figure 5.26 .

For the axially inhomogeneous films it is not so easy to quantify the effect. The reason is that one usually finds that either the fast or the slow diffusion is very dominant. In order to get statistically more reliable information about the diffusion coefficients in the slower and the faster region, a total of six diffusion videos was analysed for one film. However, there was still no distribution showing two good Gaussian peaks that could be assigned to the two diffusion regimes. Therefore the step lengths were analysed. The occurrence of the different step lengths is shown in figure 5.27. One can clearly see that it is not a simple exponential decay for a random walk with one diffusion coefficient as described by equation (3.5). However the assumption of two different diffusion coefficients (leading to a double exponential decay of the probability of the step length) present in the film leads to a reasonable fit. The obtained diffusion coefficients are $0.9 \mu \mathrm{m}^{2} / \mathrm{s}$ for the slow diffusion and $3.4 \mu \mathrm{m}^{2} / \mathrm{s}$ for the fast diffusion. The fast diffusion coefficient is slightly decreased compared to the diffusion coefficient of 90.4 averaged over the whole film obtained in section 5.2. This is understandable as here only the inner layers of the film are measured. As the surface layers are expected to have a significantly 


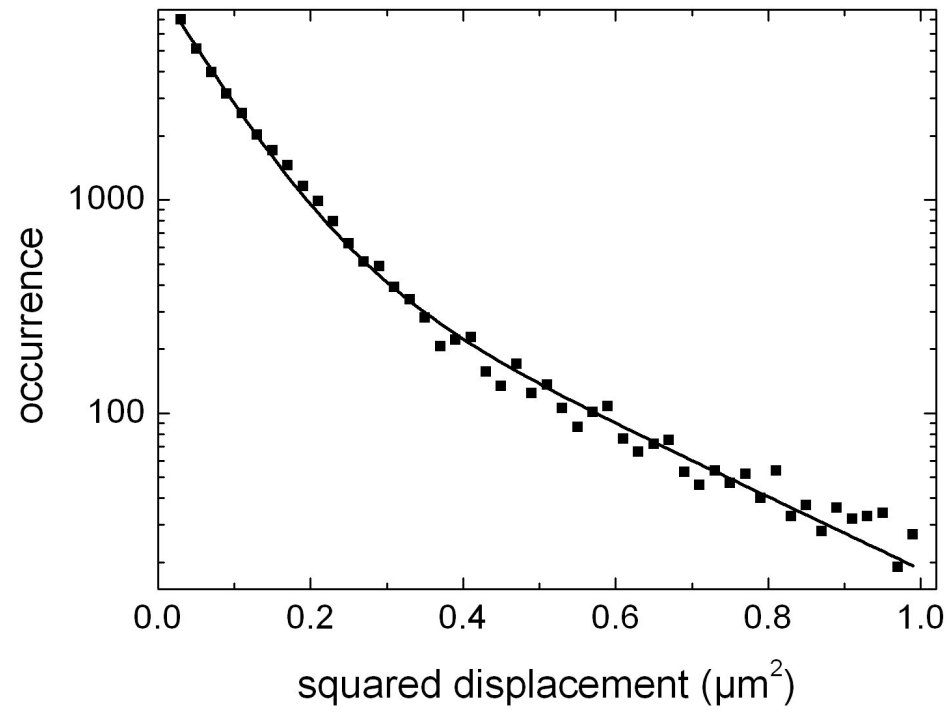

Figure 5.27: Step length distribution for an axially inhomogeneous film with a double exponential fit of the data.

higher diffusion coefficient than the middle ones (see section 5.1.1), the measurement of the inner layers has to lead to a slower measured diffusion.

On the contrary the slower diffusion appears slightly faster than obtained before on average for the hexatic phase. For this phase, one is dominantly measuring the diffusion close to the air interface which is faster than the average of the film. This explains why the difference observed in this axially inhomogeneous films is found to be a bit less than the one order of magnitude that was found for the transition of the whole free-standing film.

The evidence of two distinct diffusion coefficients in the film shows the strong tendency of the molecules to avoid phase boundaries. Already during one single step the molecules travel for several hundred nanometers. The fact that during this time the molecules do not explore the entire film with a thickness of less than 20 nanometers can hardly be explained by the normal anisotropy of the diffusion, as the usual difference between the two diffusion coefficients is not more than a factor of 2. More importantly, the observation of whole trajectories that appear to be within the same phase (shown in figure 5.26) shows a clear confirmation of the statistical facts. Together with the above explained finding that in laterally inhomogeneous films the molecules stay in their phase, the phase boundary appears to introduce a rather strict limitation on the movement of individual molecules. 



\section{Chapter 6}

\section{Ultrathin films of $8 \mathrm{CB}$ on silicon substrates}

The second chapter of the results deals with thin $8 \mathrm{CB}$ films on silicon substrates. As we will see, this system offers some outstanding features, combining mobility on molecular level with structural stability. It will be presented which kinds of structures are formed in the case of a spin-coated film and how the structures can be manipulated by using contact mode atomic force microscopy.

\subsection{Creation and properties of ultrathin smectic films on sil- icon substrates}

\subsubsection{AFM measurements of the surface structure}

If one deposits a thin film of $8 \mathrm{CB}$ on a silicon substrate, the film is subjected to an inner stress as it imposes conflicting boundary conditions: The substrate favours a planar alignment of the liquid crystal while on the free surface a homeotropic alignment is induced. The reaction of the film to that depends on the film thickness which means on the space that is available to fulfill the boundary conditions by changing the molecular orientation. For films with thicknesses, of around one micron the smectic layers bend (splay deformation of the director field) and hence tilt the orientation with increasing distance from the solid interface [30]. If the film is one order of magnitude thinner, there are hints that the orientation is changed with the help of an intermediate nematic layer close to the silicon [68, 135]. This is advantageous as the nematic 
state allows for bend deformations in the director field which enables larger gradients in the molecular orientation. For very thin films of only three molecular layers, the structure is found to be one planar monolayer on the surface and a homeotropic double layer at the air interface as shown in figure 2.9. For the region between $100 \mathrm{~nm}$ and three molecular layers $(4.1 \mathrm{~nm})$ not much is known about the inner structure of the film. One of the intentions of this work is to fill this gap that is still present.

The stability of thin $8 \mathrm{CB}$ films on silicon substrates has been under debate as already explained in chapter 2.5.1. However, we find the films to be stable regardless of the number of smectic layers (for $8 \mathrm{CB}$ one smectic layer corresponds to a molecular double layer) when it is spin-coated from a toluene solution. The concentration of $8 \mathrm{CB}$ in toluene was varied in a range between 0.2 and $5 \mathrm{mg} / \mathrm{ml}$. As the density of $8 \mathrm{CB}$ is around $1 \mathrm{~g} / \mathrm{cm}^{3}$ [80], this corresponds to roughly 0.02 to 0.5 volume percent. All the prepared films appeared homogeneous under an optical microscope and so the measurements had to be done using AFM. The first observation is that for the lowest concentrations smaller than $0.6 \mathrm{mg} / \mathrm{ml}$ there are no structures observed on the surface. For larger concentrations, regions with distinct height differences are found.

In the case of incomplete layers, two different kinds of surface structures can be distinguished. For a small coverage of the topmost layer it consists of isolated "islands" of the regions with the largest number of layers. In the case of a large portion of the topmost layer being covered, the formation of "pores" in the films is observed. Both kinds of structures are shown in figure 6.1

An outstanding property of all the observed structures is their stability in time. As the material is still liquid, one would expect a relaxation of the holes and islands to a circular shape in order to minimise the line tension of the edge and a merging of different regions. This kind of behaviour is observed for thin $8 \mathrm{CB}$ films on water interfaces [3] and for all freestanding films [40]. However, the structures presented here appear to be inherently stable in time. In contrast to the stability in our films, for the spreading of $8 \mathrm{CB}$ on silicon substrates it was found that the leading edge of the spreading droplet moves with a velocity of around two to three nanometers per second when connected to a material reservoir [37]. The only difference in our system is the absence of any directed motion. Apparently in this case the structures are stabilised by a surface memory effect [136]. It was also reported in literature that this surface memory effect might cause adsorption of the layers directly at the surfaces [137]. This, however, is not the case for our samples as will be shown later in section 6.3, as diffusion was found to be of the order of $1 \mu \mathrm{m}^{2} / \mathrm{s}$. 

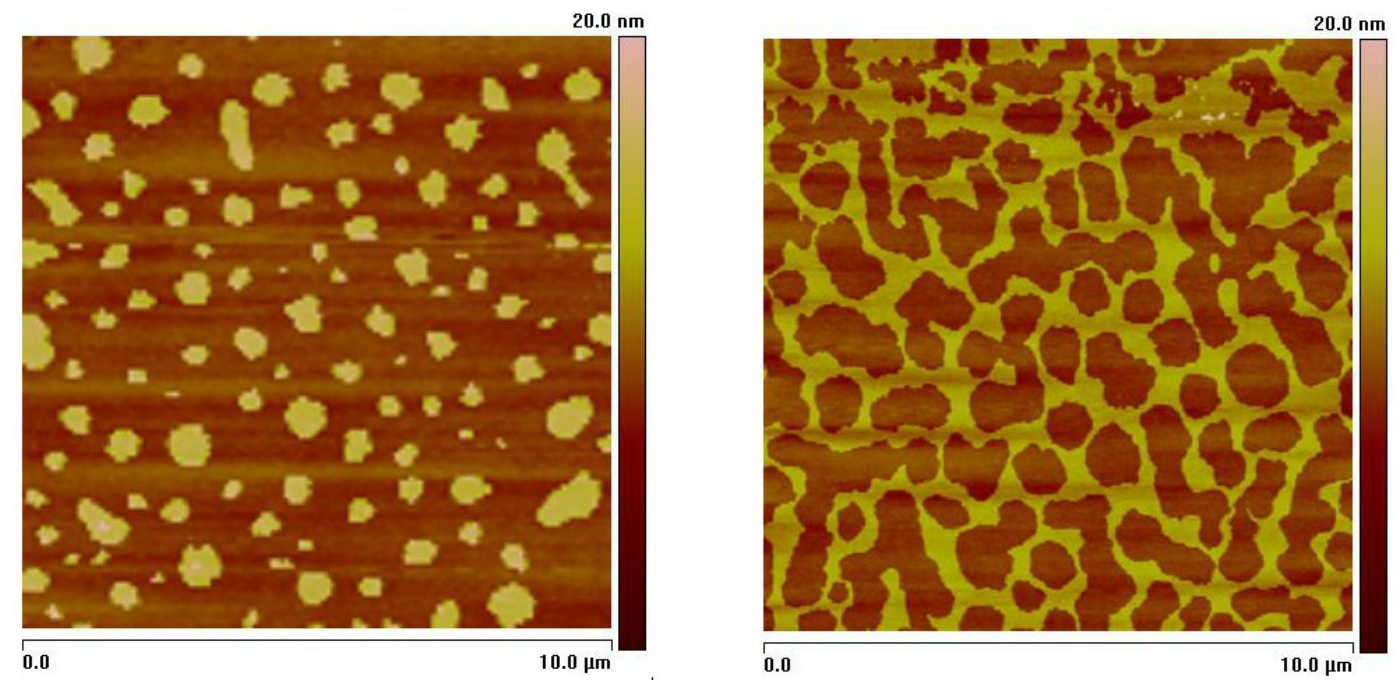

Figure 6.1: Examples for surface structures of $8 \mathrm{CB}$ with isolated islands on the left side (prepared with an $8 \mathrm{CB}$ concentration in the spin-coated solution of $0.8 \mathrm{mg} / \mathrm{ml}$ ) and porous structures on the right side $(c=1.1 \mathrm{mg} / \mathrm{ml})$. Both structures have a film thickness of three molecular layers in the higher regions as we will see later.

Owing to the stability, it is also possible to study the successive filling of the next layer on top of a completed one. It is then observed that the building up takes place nearly independent of the number of underlying layers. A sequence of samples showing the consecutive structures is shown in figure 6.2. First, for small coverages, separated islands appear. For larger amounts of deposited material the islands grow to elongated structures until they get connected. This gives rise to a structure that can be considered as a two dimensional porous structure. Upon further increasing the amount of deposited material, the holes in the structure (meaning the isolated regions where the film thickness is one layer less than in the rest) shrink until one gets the full coverage again. Then the same sequence is found for the next layer.

In order to characterise the film thickness more precisely, the coverage of the top layer can be determined by a simple binarisation of the AFM image which gives the portion of coverage. For this, a simple MATLAB code shown in the Appendix was used. Figure 6.3 gives the binarised images of the topography scans from figure 6.1 as examples. It is evident especially from the image on the right that the distinction between higher and lower regions is not perfect. The reason for this is that all the AFM images have to be smoothened as the measured hight differences are only a few nanometers over a length scale of 10 microns. This smoothening invariably leads to artifacts as the features size of the structures is not so much 


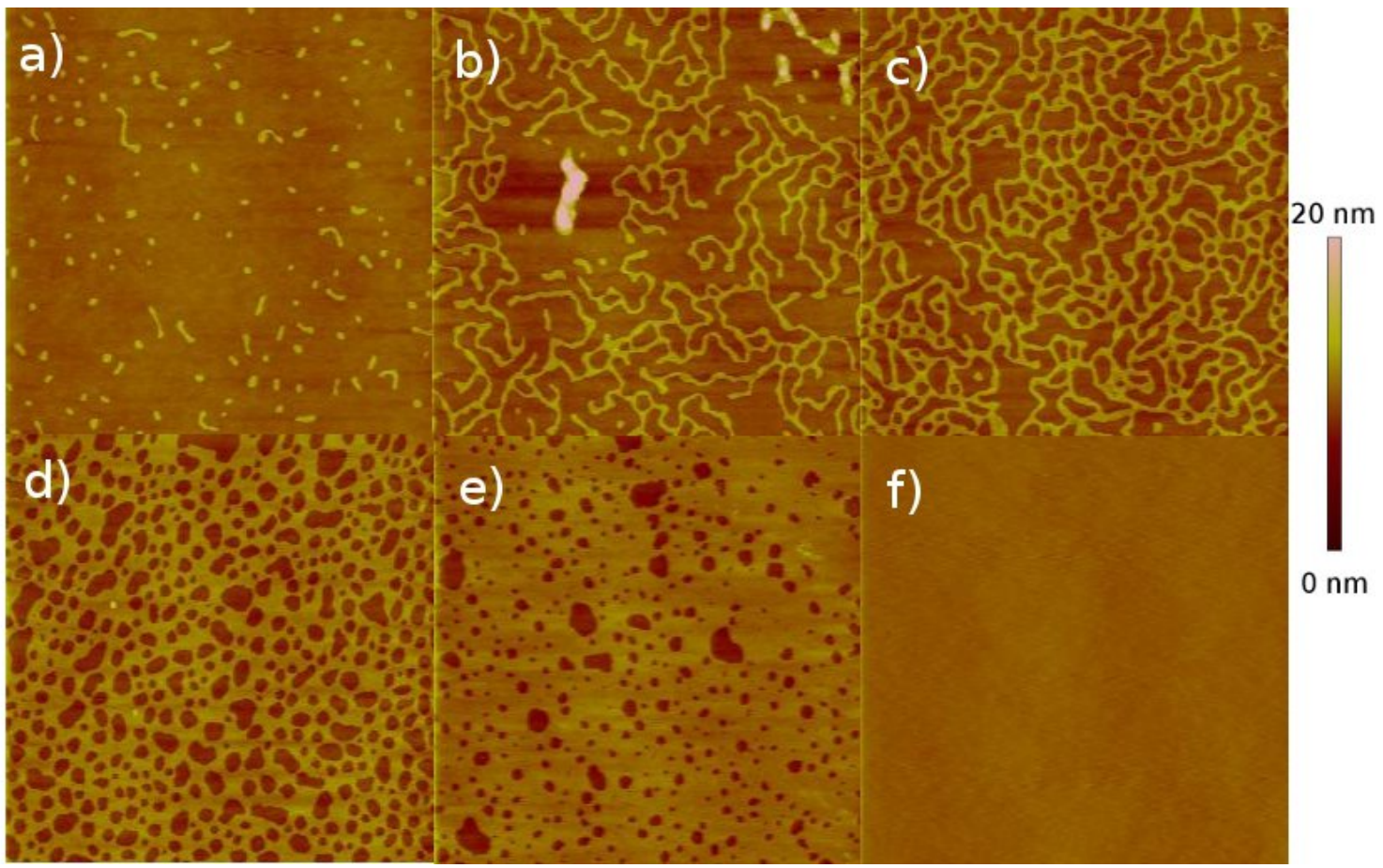

Figure 6.2: Six samples with different concentration in the spin coated solution show the consecutive steps in the creation of a new layer. The used concentration were (a) $2.8 \mathrm{mg} / \mathrm{ml}$, (b) $3.0 \mathrm{mg} / \mathrm{ml}$, (c) $3.2 \mathrm{mg} / \mathrm{ml}$, (d) $3.4 \mathrm{mg} / \mathrm{ml}$, (e) $3.6 \mathrm{mg} / \mathrm{ml}$, and (f) $3.8 \mathrm{mg} / \mathrm{ml}$. Each image shows an area of $20 \times 20 \mu \mathrm{m}^{2}$

smaller than the image size so that the real surface structure interferes with the unwanted drift that is supposed to be smoothened.

With the help of the binarised images it becomes possible to caracterise the number of layers more precisely than by a natural number. As the structures are subsequently build up, one always knows the number of underlying completed layers, although the individual image only measures height differences of the surface topography. Then the number of layers is given by the number of underlying layers plus the surface coverage. The resulting dependence of the absolute number of molecular layers of the film on the concentration of $8 \mathrm{CB}$ in the spin-coated solution is given in figure 6.4. The plot also shows vertical lines for the transition from the island structure to the porous structure and the completion of a layer respectively. The distinction between the structures is done simply by eye. One can now determine the threshold coverage necessary for the transition. It is found to be only slightly dependent on the number of underlying layers and is always at a coverage of around $35 \%$ of the top layer. 

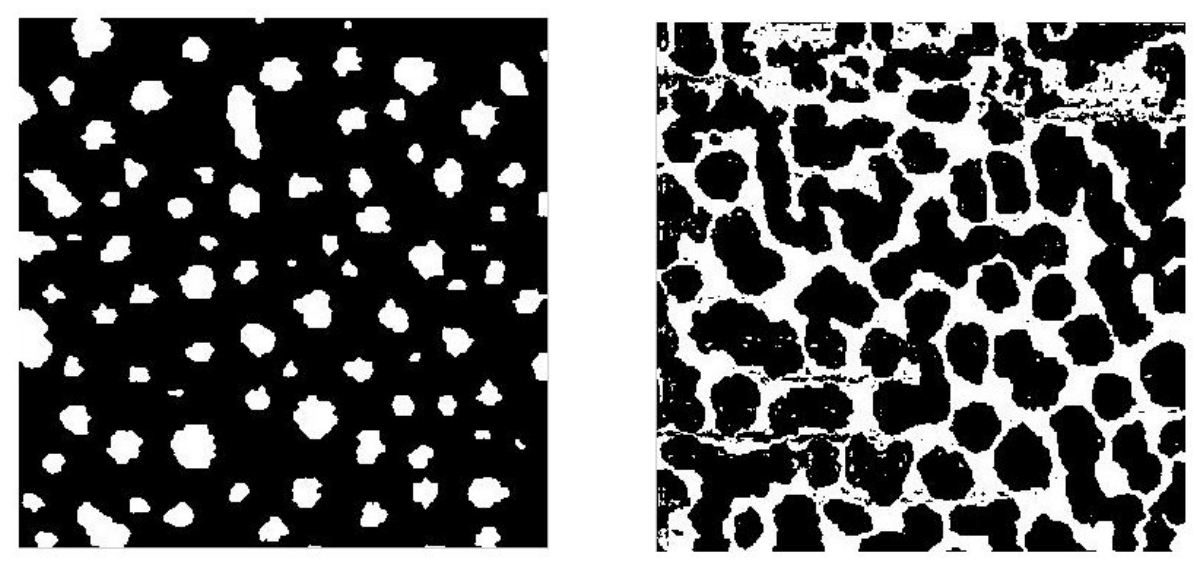

Figure 6.3: Examples for binarised images: For the surface structures shown in figure 6.1, the images were binarised to determine the coverage of the surface layer. In the left case a coverage of $16.5 \%$ and in the right case $35.7 \%$ was found.

It was tested how homogeneously the structures were formed. For this purpose the coverage was measured on different spots of the surface in order to see how big the fluctuations are. The maximum distance of the spots was around $4 \mathrm{~mm}$ (still staying in the central region of the sample of size $1 \mathrm{~cm}$ ). For a film prepared with a concentration of $3.6 \mathrm{mg} / \mathrm{ml}$, which corresponds to around 2.8 layers, 15 different AFM images were analysed. The standard deviation of the measurements was found to be only 0.022 layers. This means that the error one can estimate for the measurements of figure 6.4 mainly results from the error in the concentration of the spin-coated solution. Here the problem is that smectic liquid crystals cannot be handled with micropipettes easily because of their high viscosity. That is why they always need to be heated up for the preparation of the mixture. Because of the complications, the error is larger than the $1 \%$ from the supplier specification of the micropipettes and can be estimated to be around $5 \%$.

The existence of steps for the changes of the film thickness in a sample can be expected for a smectic liquid crystal where the inner structure consists of layers. To show that the film thickness differences really correspond to one smectic layer (i. e. one molecular double layer in case of $8 \mathrm{CB}$ ), the cross section has been measured to compare our step height with the thickness of $3.16 \pm 0.04 \mathrm{~nm}$ which is the layer spacing in bulk 8CB material [109]. However, AFM topography images actually give the height of constant amplitude which means for the sample the height of equal damping of the cantilever. As a consequence, for a sample with 


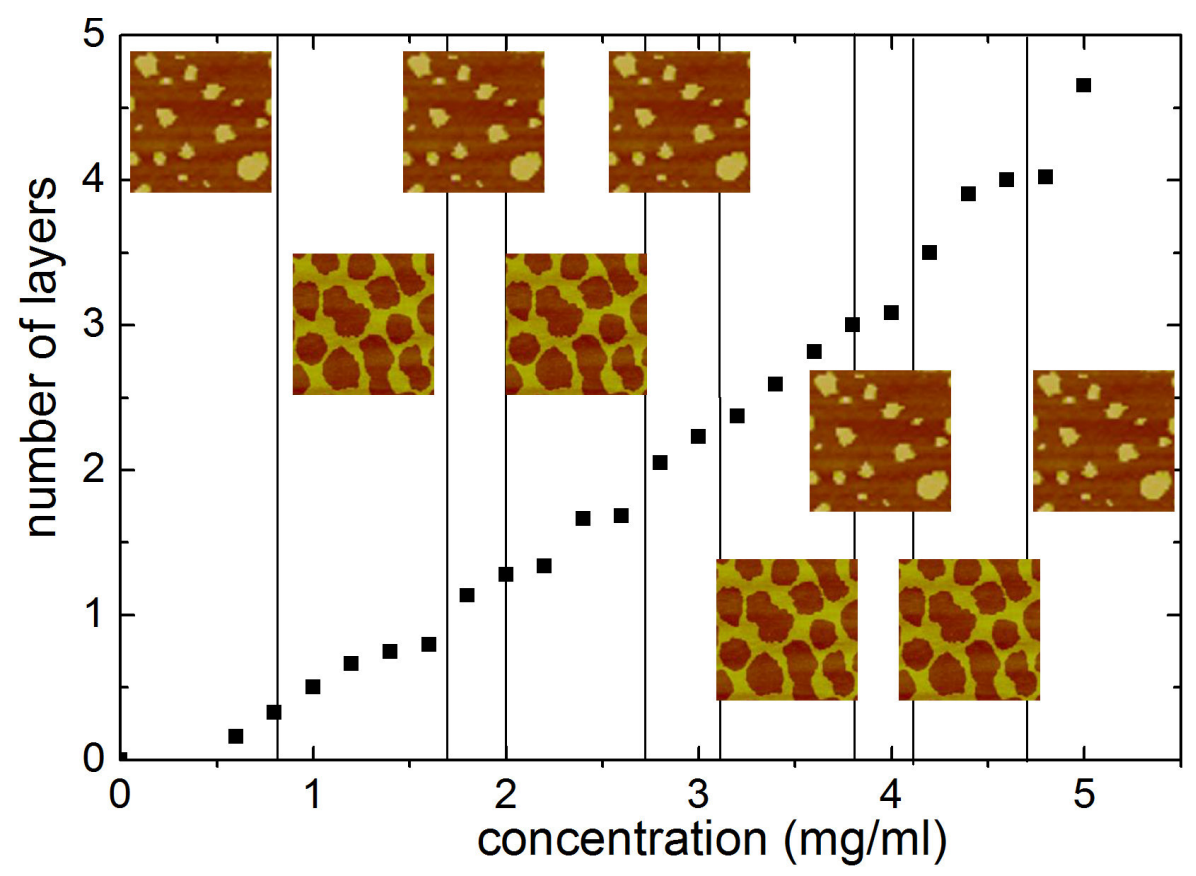

Figure 6.4: Number of deposited layers depending on the $8 \mathrm{CB}$ concentration in the spincoated solution. The vertical lines give the transition between the island structures and the porous structures.

heterogeneous surface properties one always gets a difference between the apparent height measured by AFM and the real feature height [138], depending also on the scan parameters used. This was also observed for the measurement of step heights of 8CB layers close to silicon surfaces in a similar way [91]. The effect can qualitatively be seen in figure 6.1 where both images are supposed to show the same height difference between the lower and the higher regions; however, the image with the islands visibly has a higher apparent step height than the image with the pores.

The difference in the surface properties should be the strongest for the thinnest films and becomes less pronounced the more smectic layers are under the scanned surface. Because of this, the step height was measured for the example of two completed layers (shown in figure 6.5). The step height was found to be $3.3 \pm 0.2 \mathrm{~nm}$ which agrees well with the mentioned bulk layer spacing. Hence, we can conclude that the observed steps correspond to one smectic layer as expected.

Smectic liquid crystals are known to show a strong surface stabilisation confirmed by a shift to higher phase transition temperatures both at the substrate surface [137] and at the free 


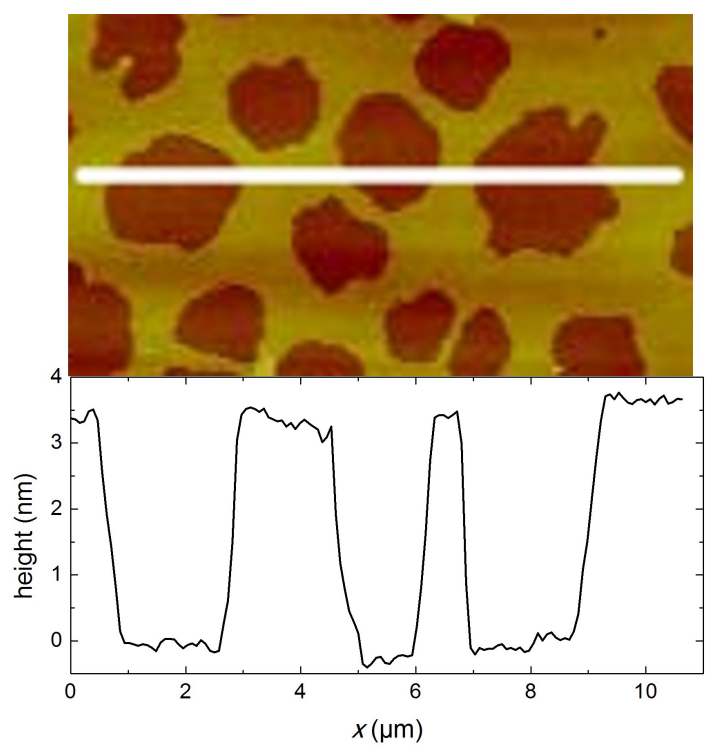

Figure 6.5: Cross-section along the white line in the image. The film was prepared with a concentration of $3.4 \mathrm{mg} / \mathrm{ml}$ and shows a step height of $3.3 \pm 0.2 \mathrm{~nm}$.

surface [48]. The same property can be observed for the described films. To decide whether a film is still in the smectic phase, the presence of the step-like changes in film thickness was used, as this is a typical smectic property. It is always observed that the disappearance of the smectic steps coincides with dewetting and the visible formation of droplets on the surface. For the film with a thickness of less than one complete layer, it was found that the film could be heated up to $58^{\circ} \mathrm{C}$ without noticeable structural changes (it should be reminded that the bulk phase transition temperature to the nematic phase is at $33.7^{\circ} \mathrm{C}$ and to the isotropic phase at $40.5^{\circ} \mathrm{C}$ ). For one completed underlying layer the phase transition was found to be around $38^{\circ} \mathrm{C}$ and for two underlying layers around $37^{\circ} \mathrm{C}$. However, the behaviour is not completely uniform across the whole sample. For example, it is found that for one underlying layer the first dewetting droplets appear at around $33^{\circ} \mathrm{C}$ while over most of the sample the surface structure remains unchanged for a time span of at least one day. The observed inhomogeneous dewetting can most probably be attributed to imperfections on the substrate surface that act like condensation nuclei. This means that the above stated temperatures are valid for a clean silicon surface. The behaviour of the droplets is considered separately in section 6.2 .

As usual for AFM tapping mode, the topography image was captured together with the phase image of the surface. The phase image supplies information about the surface properties of different regions of the sample, or more precisely of the energy dissipation of the surface 

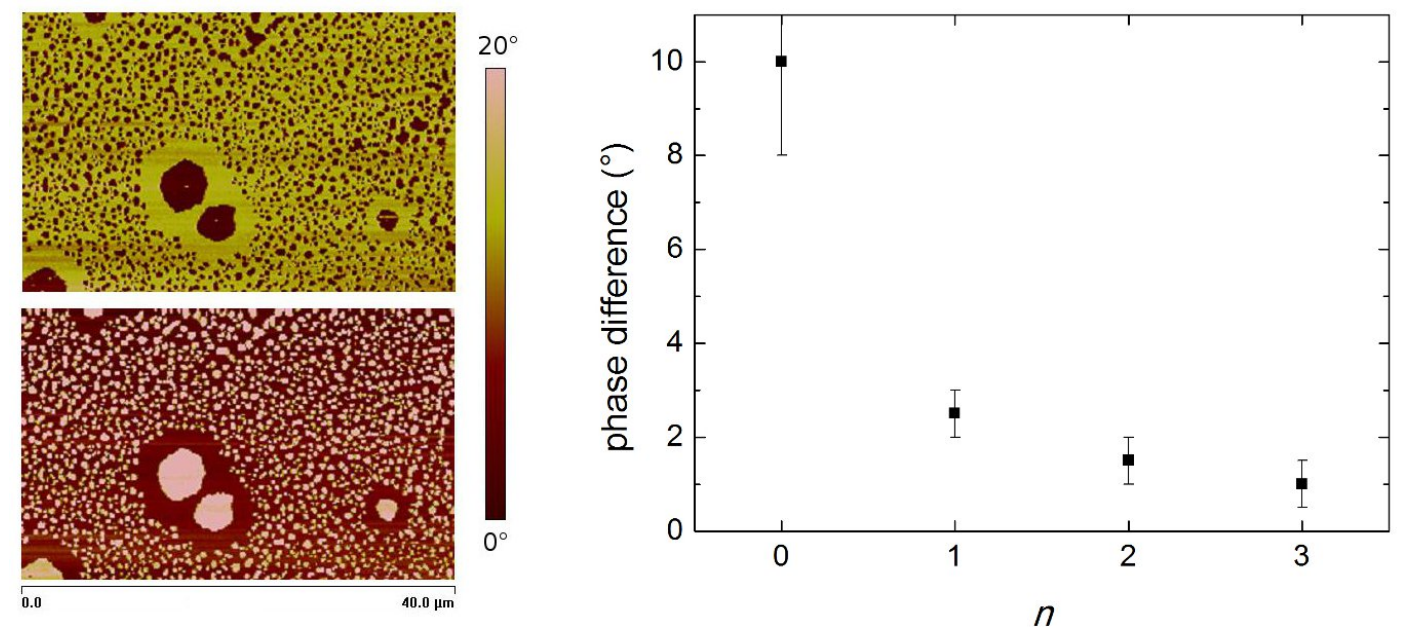

Figure 6.6: left: Example for the inversion of the phase contrast caused by a change in scanning parameters with weak tip-surface interaction on top (the "normal" scanning conditions) and strong interaction at the bottom. right: Phase difference between different molecular layers. $n$ is the number of underlying layers and the phase difference is measured compared to one layer below. The error bars represent the fluctuations found between different samples.

[139]. However, the absolute values of the phase images are very difficult to interpret as this requires precise control of the scanning parameters [140]. For example, it is even possible to invert the contrast of a phase image by drastically increasing the tip-surface interaction as shown in figure 6.6

For the measurement of the soft surface material of our sample, it was most crucial to achieve a proper imaging without disturbing the inherent features. The scan parameters primarily had to be adapted to this requirement. Hence, the absolute values of the phase images mainly serve for a qualitative comparison. Still the used scanning parameters were similar amongst each other in the way that the surface interaction was intended to be at minimum and the images were all taken in the attractive mode of the AFM (which simply means that the attractive forces between tip and surface outbalance the repulsive forces). The phase difference found between the higher and the lower regions of our samples depending on the number of layers are given in figure 6.6 .

Generally speaking, the phase image provides information about the softness of different regions of the sample. This allows for the imaging of changes in the surface porperties regardless of any existing height changes. In the field of liquid crystals, examples for possible applications of this technique are the imaging of different domains in liquid crystal polymers 

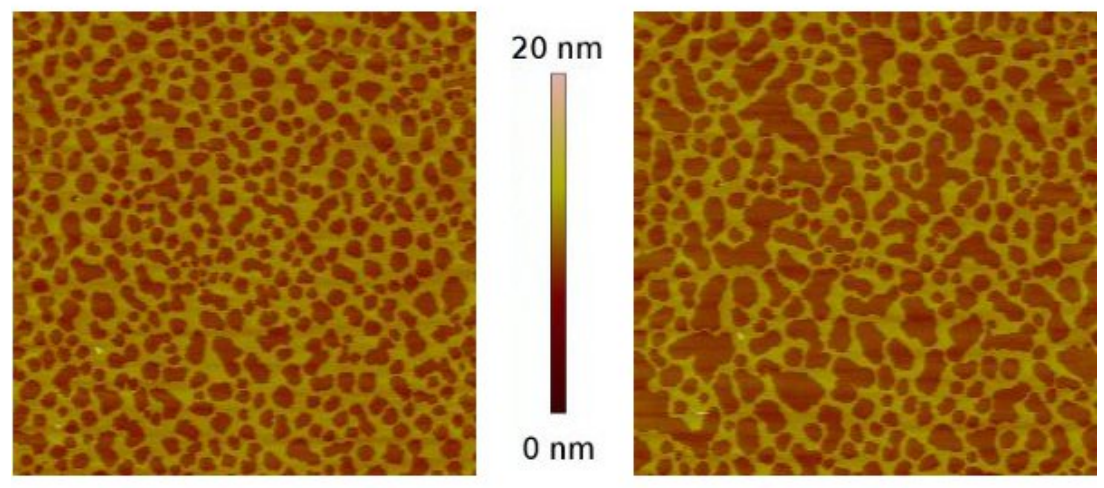

Figure 6.7: Changes in the surface structure after 5 days: The left image shows a film prepared with a concentration of $1.0 \mathrm{mg} / \mathrm{ml}$ directly after preparation. The right image shows the same spot after a waiting time of 5 days.

[141] or the identification of defects on the surface [142]. The general tendency is that harder material shows a brighter contrast. It was shown that the phase angle increases with increasing viscosity [140]. For Langmuir-Blodgett films on silicon substrates, the layers closer to the surface would appear brighter because of the surface stabilisation [143]. It is therefore surprising that we observe exactly the inverse contrast for scanning with low interaction. This means that in our films the layers closer to the surface turn out to be softer. This interpretation is also in agreement with the finding that the apparent step height is always larger than the real one, which also indicates that the lower regions are softer [138].

As more and more layers are added to the surface, the surface properties on both sides of the step should become more similar. It is therefore obvious that the phase difference has to strongly decrease on increasing the number of layers.

The stability of the surface structures in time for several hours has already been mentioned. Also it was tested to which extent the structures change over days. The difference of the topography after five days is shown in figure 6.7. There are no major structural changes. Some of the pores are found to have merged but no general relaxation of the structure can be seen. A general decrease of the amount of material can be observed. The calculated surface coverage is reduced from $52 \%$ to $41 \%$. As the film was not kept under closed atmosphere, this effect might probably be caused by evaporation of the liquid crystal. 
The reasons for the formation of the observed structures remain largely unclear. However, it is interesting to notice that structurally the porous films look very similar to the dewetting patterns observed for the liquid crystal $5 \mathrm{CB}$ when heated to the transition temperature between nematic and isotropic state [144]. In that case, the lateral and axial dimensions of the structures are around one order of magnitude larger than for our films. Still one might speculate that the observed structures could be caused by an instability during the evaporation process of the spin-coating. The changing concentration of $8 \mathrm{CB}$ will give rise to phase transitions that in turn could evoke the structures that are surface stabilised afterwards.

\subsubsection{Ellipsometry measurements of the film thickness}

Ellipsometry measurements were done to measure the absolute film thickness of the studied films. First of all, it is necessary to confirm that the used preparation method enables one to obtain films with an arbitrary number of layers, as the possibility to form a small number of $8 \mathrm{CB}$ layers was negated before [34, 35]. For this it is sufficient to measure the ellipsometric amplitude ratio $\tan \Psi$, as for film thicknesses that are a lot smaller than the used laser wavelength, the value of $\tan \Psi$ at the Brewster angle (this minimum will be called ellipticity coefficient $\bar{\rho}$ ) is proportional to the film thickness. The graphs for the values of $\tan \Psi$ for the first four layers are plotted in figure 6.8. Without any quantification it is directly visible that subsequent layers build up for the different samples.

Using the AFM images one can only speculate about the internal structure of the film as only the surface of the top layers is scanned. However, one would assume that the structure at the surface is always the one shown in figure 2.9 with a nearly planar monolayer on the substrate and on top of it smectic double layers with homeotropic molecular orientation. To check this expectation, ellipsometry measurements can be used, as this method is able to measure small film thicknesses with a precision below the nanometer scale. The model orientation described above leads to expected properties of the light reflected from the surface. These properties can be compared to the real properties measured by ellipsometry.

For the AFM measurements, the film thickness can be determined with the help of the coverage from figure 6.4, using the known film thickness of the first trilayer $(4.1 \mathrm{~nm})$ and the one of the double layers $(3.2 \mathrm{~nm})$ [37]. As already mentioned, no surface structure is found for concentrations below $0.6 \mathrm{mg} / \mathrm{ml}$. This hints to the fact that for smaller concentrations only a monolayer is partially present on the surface. For this surface layer no structure is found in consistence with both experiments [36] and modeling [145], suggesting a non- 


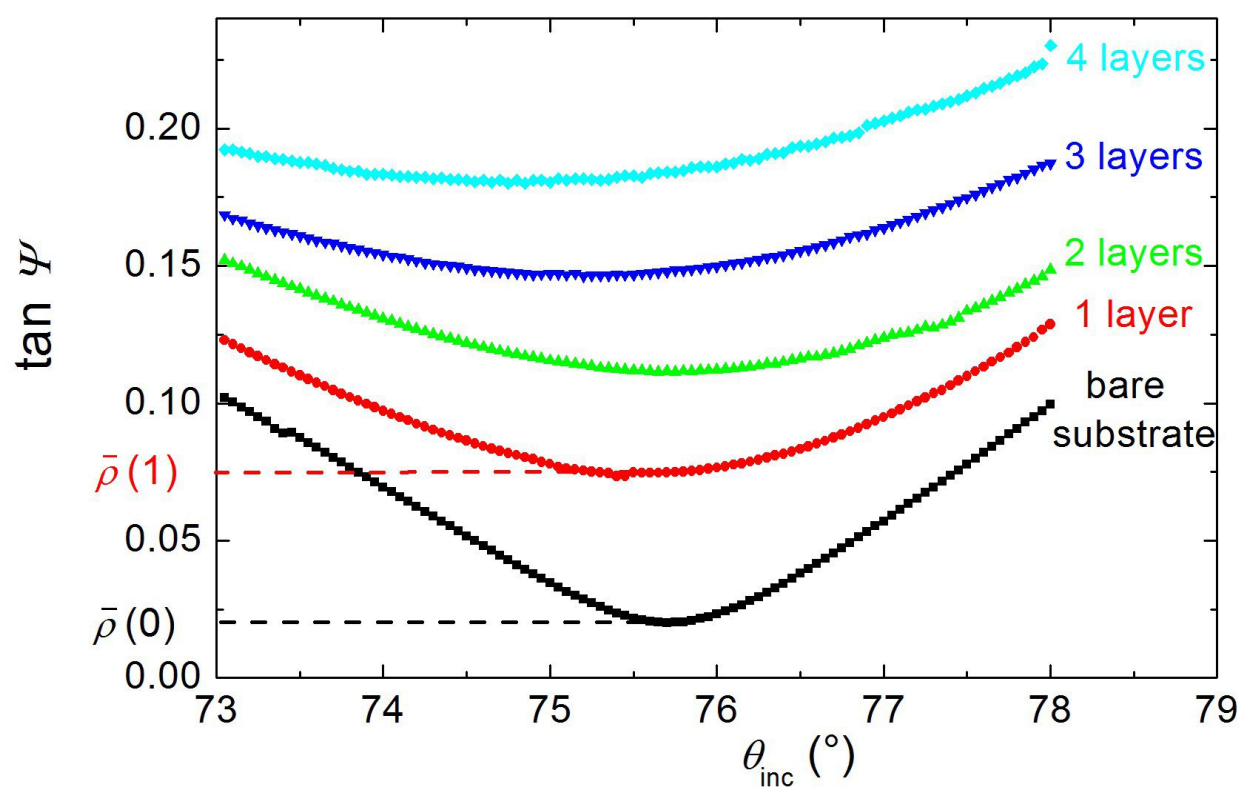

Figure 6.8: Amplitude $\tan \Psi$ for the first four completed layers judged from the AFM measurements. The data shows a clear build-up of the layers given the proportionality between the film thickness and $\bar{\rho}$ for small film thicknesses.

dense ("gaseous") first monolayer in case of insufficient material for a complete layer. This means that the first observed structures represent the first double layer formed on top of the monolayer.

The quantitative interpretation of the ellipsometry data is not as straightforward as for the AFM images. Instead, the obtained data has to be compared to a model system. For a thin film of an anisotropic medium on a smooth surface this can be done using the $4 \times 4$ matrix method [102]. The refractive index for the used wavelength of $633 \mathrm{~nm}$ for silicon was set to $n_{\mathrm{Si}}=4.05-0.028 \mathrm{i}$. For $8 \mathrm{CB}$ the extraordinary index was $n_{\mathrm{e}}=1.67$ and the ordinary index $n_{\mathrm{o}}=1.51$ [81]. Additionally, one always finds a thin layer of silicon oxide on top of the silicon for all silicon wafers kept in air. The effect of this can be seen in figure 6.8 for the curve of the bare substrate. For an ideal interface the ellipticity coefficient $\bar{\rho}$ would be zero. Using the finite value of $\rho=0.02$ and the refractive index of $n_{\mathrm{SiO}}=1.47$, the thickness of this layer could be determined to be $1.6 \mathrm{~nm}$. This now enables us to calculate the theoretical values of $\bar{\rho}$ for our model with homeotropic alignment of the liquid crystal which is shown in figure 6.9

The used model slightly simplifies the real situation. The first point is that actually the first monolayer with a height of $0.9 \mathrm{~nm}$ is not homeotropic but tilted. However, the introduction 


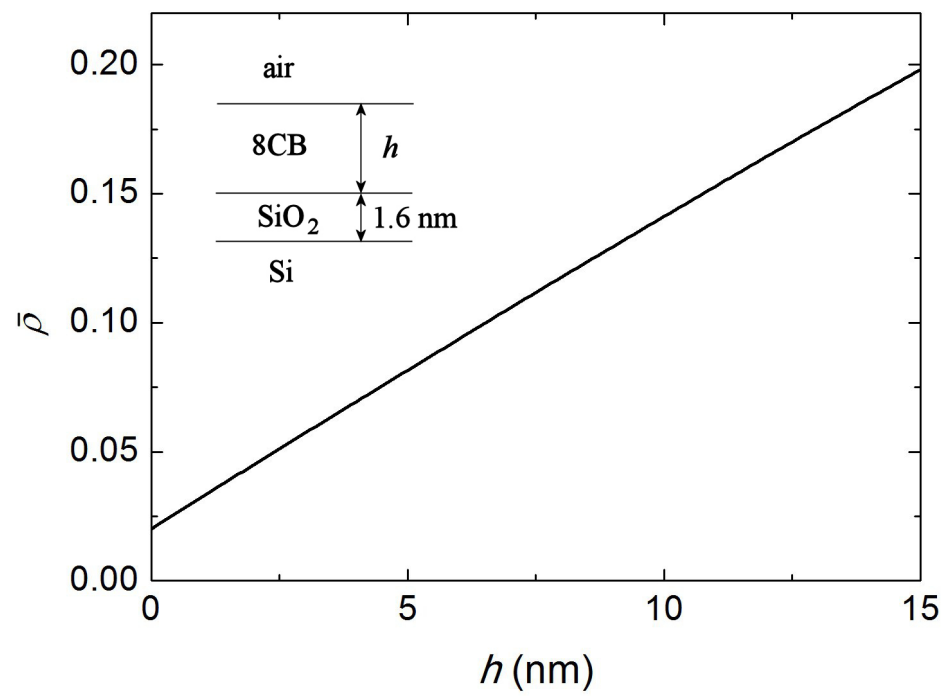

Figure 6.9: Calculated theoretical ellipticity coefficients $\bar{\rho}$ depending on the film thickness of the liquid crystal. The used model system includes homeotropic alignment of the $8 \mathrm{CB}$ molecules and a native silicon oxide layer of $1.6 \mathrm{~nm}$ thickness.

of such a layer into the model would change the ellipticity values by less than $1.5 \%$ which is less than the experimental accuracy. It can thus be neglected in our calculations. The second point is that we have an inhomogeneous surface with islands and pores and discrete changes in the film thickness instead of a continuous increase. But as for the ellipsometer an unfocused laser beam was used, the measurements average the signal over a region of around $1 \mathrm{~mm}^{2}$. The inhomogeneities are therefore not relevant for the obtained ellipticity coefficients. The discrete structure of the steps could be modelled by a smooth transition of the refractive index in the top layer, for example by a tanh function. However, also here the difference in the calculated $\bar{\rho}$ values is rather small below 0.005 , which corresponds to roughly $0.3 \mathrm{~nm}$, so that the simple model is reasonably precise for our system.

Now it becomes possible to directly compare the AFM data with the ellipsometry measurements. The calculated film thicknesses using the two methods are shown in figure 6.10. It can be seen that the two data sets agree very well. This means that the assumption of the structure, as consisting of homeotropic layers apart from the first monolayer, is strongly supported by the ellipsometry measurements. 


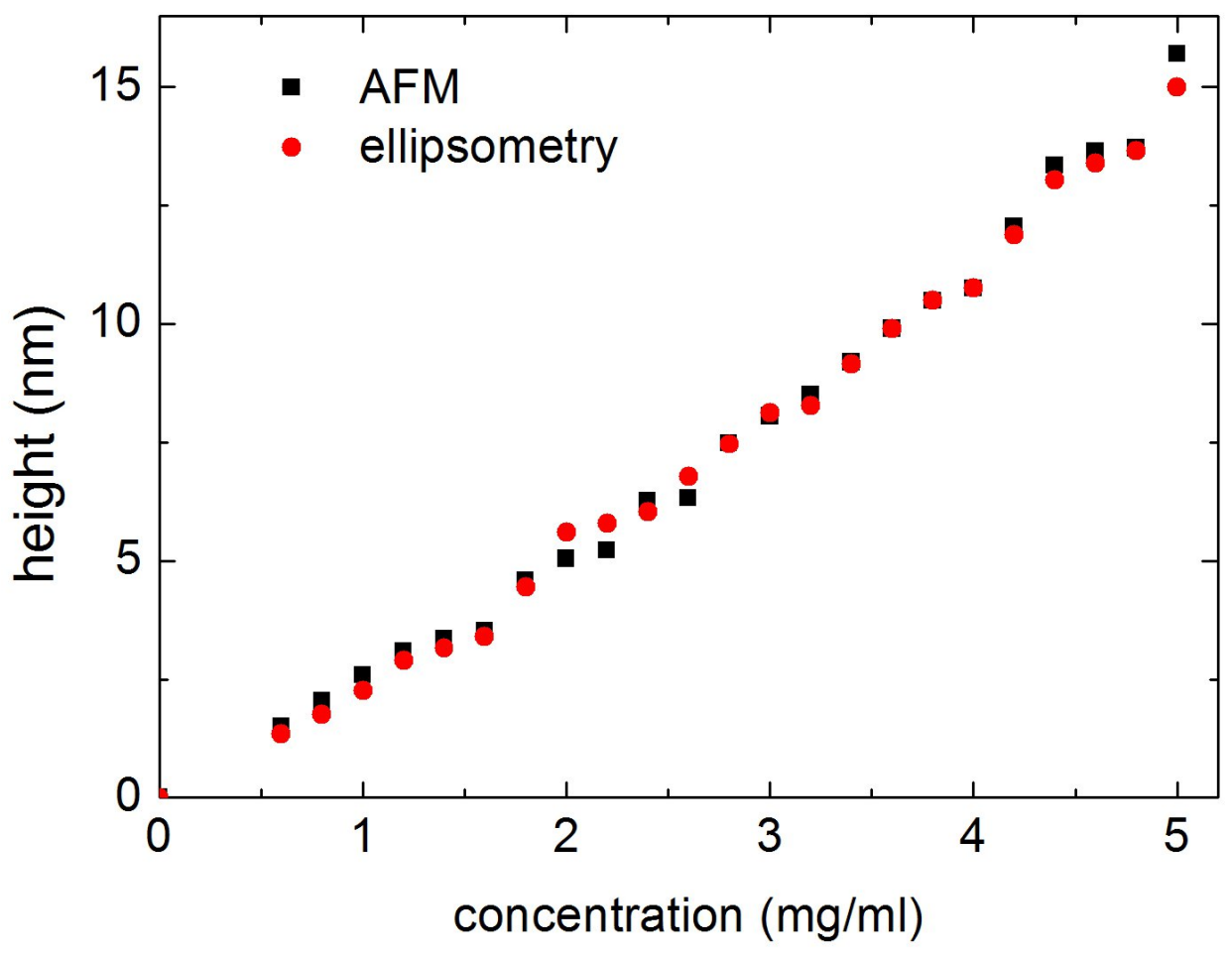

Figure 6.10: Comparison of measured film thicknesses with AFM and ellipsometry: The ellipsometry data was obtained using the $\bar{\rho}$ and the described model calculation. The AFM thickness results from the surface coverage.

\subsection{Spreading of femtolitre droplets}

As explained in section 6.1.1 the homogeneous films dewet above a certain temperature that is dependent on the original film thickness. This leads to the creation of isolated droplets with a typical volume of $10^{-15}$ to $10^{-14}$ litres. On cooling down into the smectic phase again, the droplets start to spread as the substrate offers complete wetting conditions for the smectic $8 \mathrm{CB}$. This allows us to study the spreading of a very small amount of material. When analysing this phenomenon it might be possible to bridge the gap between the spreading from a big reservoir and our stable homogeneous films. For the case of the reservoir, a spreading velocity of the leading edge of two nanometers per second is observed [37].

As the dewetting at elevated temperatures leads to a random distribution of droplet sizes, it is possible to study the spreading of drops with different numbers of layers. This is done again via AFM as this supplies the higher resolution compared to optical imaging. The minimum height of such a droplet was 8 layers while the maximum was around 64 . For all the 

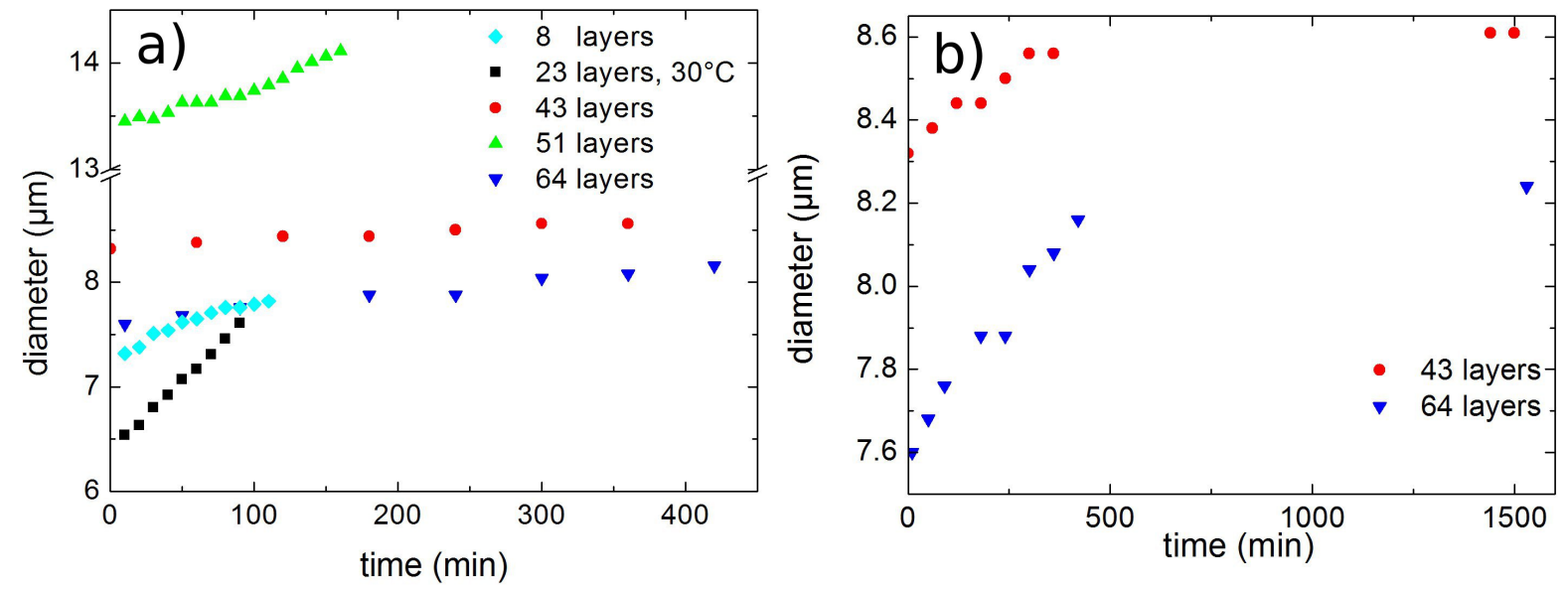

Figure 6.11: Spreading of smectic droplets with different heights. a) No apparent dependence on the droplet size can be found. Clearly faster spreading is observed for the case of the temperature being increased to $30^{\circ} \mathrm{C}$ (black squares). The number of layers is estimated from the droplet height and refers to the initial state. b) Size evolution after a longer resting time shows insignificant spreading when no scanning of the surface is carried out.

thicknesses it is found that the width of the drops increases linearly with time at the beginning as shown for several examples in figure 6.11a). The size of the droplet does not seem to affect the growth rate of the droplets. For the ones which where measured at room temperature, there is no correlation visible between the spreading speed and either the number of layers of the droplet or the width at the initial stage. Only the droplet that was spreading at $30^{\circ} \mathrm{C}$ increases its diameter significantly faster than the other ones.

The graph also shows a stronger gradient for the samples that were scanned more often in a shorter time span even for the case of 8 layers where the droplet should be surface stabilised the most. This indicates that the scanning actually influences the spreading behaviour of the droplets. This was tested by having a longer resting time of the sample and check if the previous trend is preserved or not. It is found that the size difference of a droplet between two measurements with longer waiting time is approximately the same as before for the short time between two measurements (figure 6.11 b). This would mean that at our usual temperatures of $23^{\circ} \mathrm{C}$ all the observed spreading would be caused by the interaction with the tip and there is no genuine spreading of the droplet on the time scales of hours. Also the observation that the volume is not conserved during the scanning hints to a too strong interaction of the tip and the surface which does not allow undisturbed measurements of the system. 

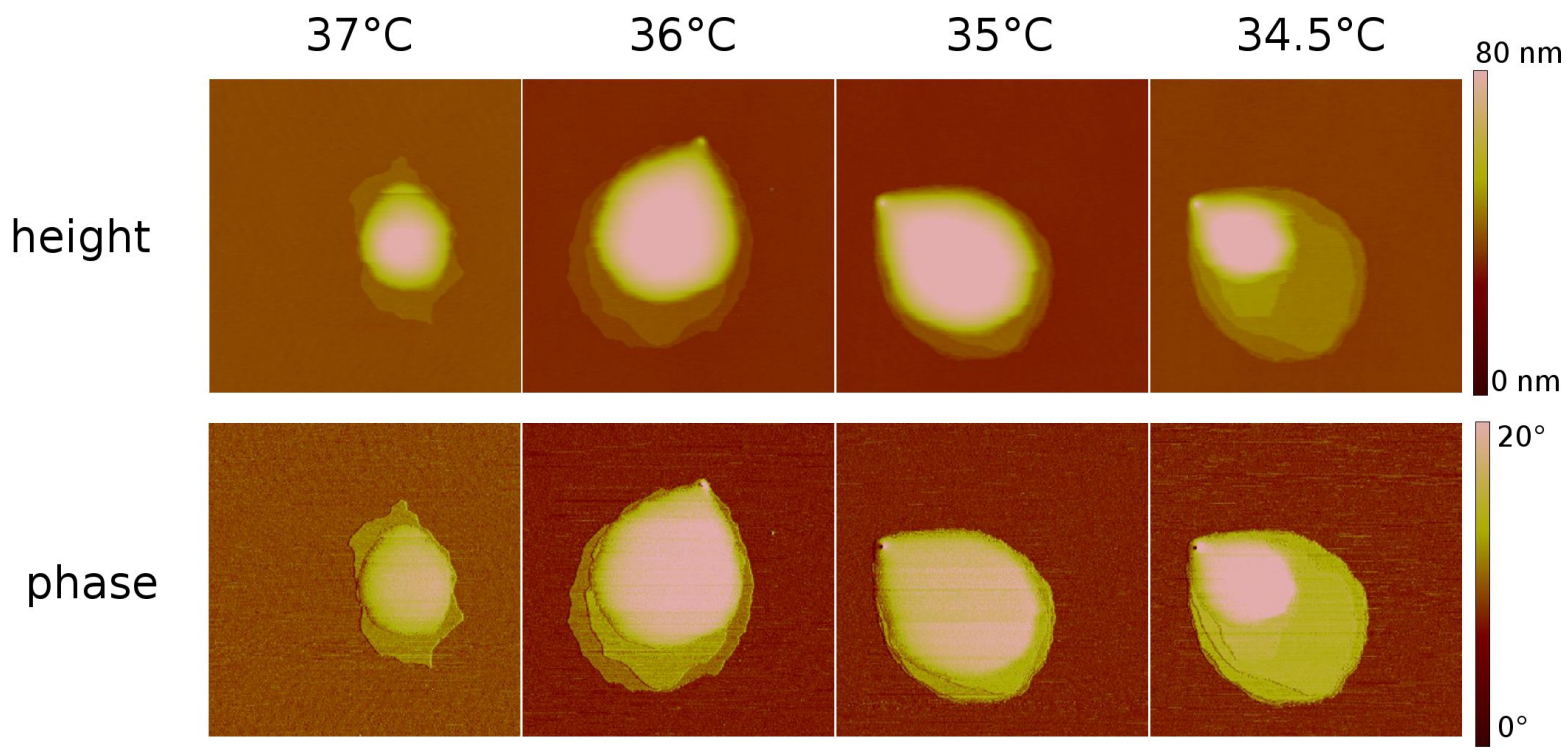

Figure 6.12: Cooling of a sample with a dewetted film. The images show the subsequent formation of the first four smectic layers close to the surface (not all the droplets are identical). Height and phase images of the samples are depicted for better identification of the steps. The color scale is valid for all the images. All images are $10 \times 10 \mu \mathrm{m}^{2}$ in size.

Additionally, it is possible to optically measure the spreading of the droplets. However, the edges in the optical images are not very sharp so that the measurement of the diameters would contain a high degree of arbitrariness.

Apart from the spreading velocity, one can also observe the smectic wetting of the surface when the temperature is slightly above the phase transition temperature from the smectic to the nematic phase $\left(33.7^{\circ} \mathrm{C}\right)$. When slowly cooling down from the isotropic phase, one finds the formation of smectic layers that spread out from the droplet. One can therefore observe the phase transition temperature for approximately the first seven layers. A series of droplets scanned upon cooling from the isotropic phase is shown in figure 6.12. In this regard it is not important that the images show different droplets as the wetting behaviour should not depend on the amount of nematic material on top of it. The images show how subsequently the smectic layers build up when cooling the sample. Usually such a smectic wetting of the surface is observed in the case of a homeotropic surface anchoring [146]. The reason is that in this case the surface induced ordering of the molecules close to the surface causes smectic layering, thereby reducing the phase transition temperature for the layers directly at the substrate surface. In our case the situation is similar despite the planar alignment of the 


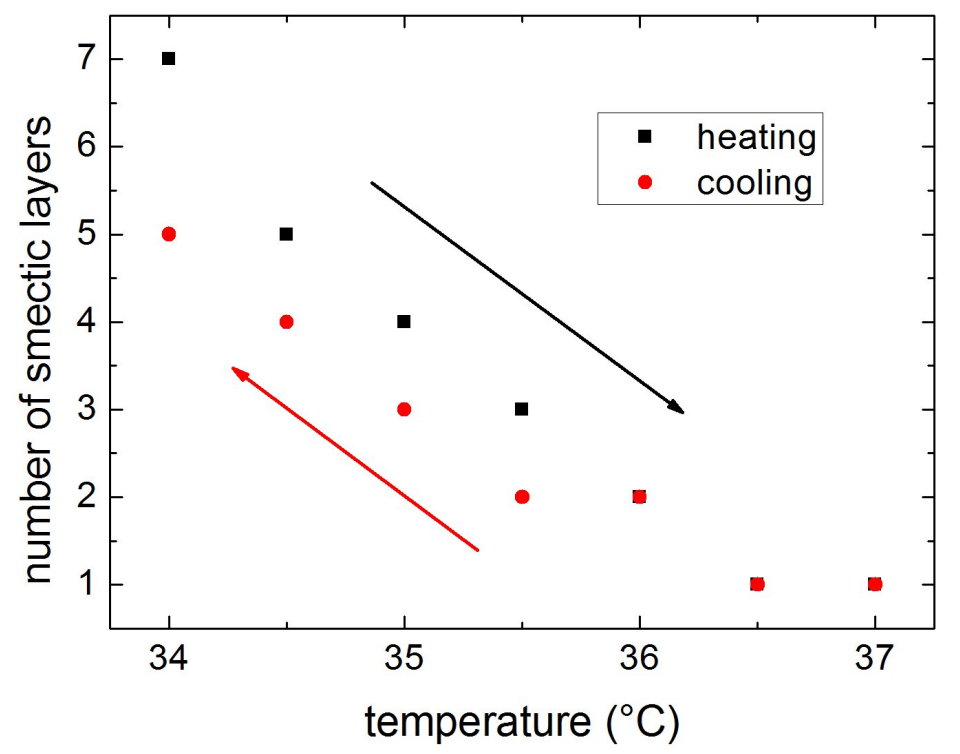

Figure 6.13: The number of visible layers depending on the temperature shows a pretransitional layering of the droplets for the cooling and the heating cycle. A small hysteresis of around $0.5 \mathrm{~K}$ is observed. For temperatures higher than $35.5^{\circ} \mathrm{C}$ the number of layers is identical for cooling and heating.

surface. The reason can be seen in the molecular arrangement close to the substrate from figure 2.9. Already the first smectic layer on top of the monolayer exhibits homeotropic orientation. It was shown in section 6.1.2 that this orientation also holds in the case of more than one layer.

After reaching the bulk smectic temperature range one can again increase the temperature, studying the gradual disappearance of the smectic layers. However, the temperatures for the appearance of the layers upon cooling and for the disappearance upon heating are not completely identical as shown in figure 6.13. Such hysteretic behaviour is also observed for other kinds of surface induced transitions [101]. However, we cannot completely exclude the possibility that the droplets are not yet in equilibrium.

\subsection{Single molecule diffusion in thin films on substrates}

For the experiments studying the diffusion of dye molecules in thin films of $8 \mathrm{CB}$, the silicon substrates with native oxide cannot be used. As mentioned before, in close proximity of the silicon the fluorescence gets quenched and the tracer molecules become invisible. For this reason silicon with a $100 \mathrm{~nm}$ thick layer of silicon oxide is used. The disadvantage of using 


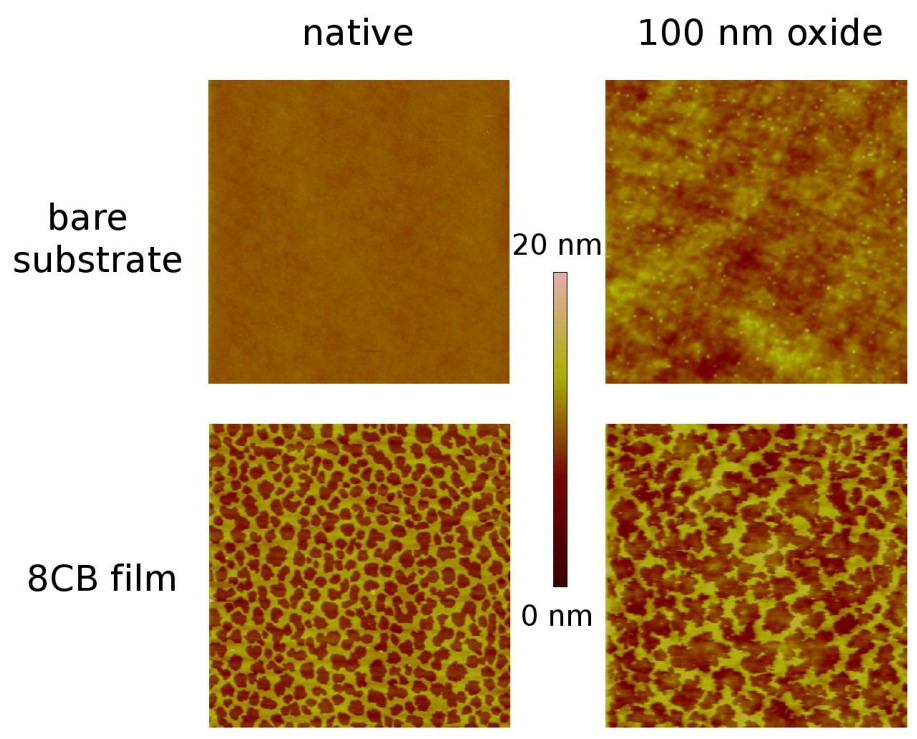

Figure 6.14: Comparison of silicon substrates with native oxide layer and $100 \mathrm{~nm}$ thermally grown oxide. The substrates with the native oxide appear a lot smoother than the ones with thick oxide layer. The structure of the $8 \mathrm{CB}$ film is more rounded compared to the frayed structures on substrates with thermal oxide. All images have a size of $20 \times 20 \mu \mathrm{m}^{2}$

such substrates is the increase of the surface roughness of such substrates caused by the process of thermal growth of the oxide layer. This results in the formation of structures that appear deformed compared to the ones found on the flat native oxide from figure 6.1. Nevertheless, all the other properties of the structures concerning temporal stability and reproducibility are the same. The comparison of the structures together with images of the bare substrates are shown in figure 6.14. The optical discrepancy of the surface can be quantified as simplest approach by the root mean squared (RMS) value. For the native oxide one finds $0.40 \mathrm{~nm}$ and for the thermal oxide a value of $1.14 \mathrm{~nm}$ is obtained.

One of the initial intentions was to use the porous films with less than one smectic layer as model systems for two-dimensional diffusion in a porous medium. It is generally assumed that the first monolayer is attached to the surface by hydrogen bonds between the silanol group of the substrate surface and the cyano group of the $8 \mathrm{CB}$ molecules. This would mean that the diffusion cannot take place in this solid-like layer but only in the regions which are covered by one smectic layer. Such a diffusion is of fundamental interest as it would be a model system for a two-dimensional porous medium with porosities of up to $65 \%$ (with the usual 
definition of porosity as being the ratio between the void regions to the total surface). The idea was to use single molecule tracking to study the properties of the diffusion in such an environment. As a first approach for this it should be possible to obtain a "diffusional map" of the surface topography. As the localisation accurracy is a lot better than the typical pore size of one micron, one should see the "holes" in the diffusional map as the regions that are never visited by the diffusing molecules. This would correspond to the holes in the smectic layer. However, it was never possible to identify regions that were avoided by the diffusing molecules. A reason might be that sometimes a slow diffusion is found in the surface layer despite the strong hydrogen bonds [147]. This might also be the case for our first monolayer as it will be explained in the next paragraph.

Because of the observations described above, we have to restrict ourselves to the analysis of the diffusion in the completed layers. The analysis of films on substrates is a bit more complicated than in free standing films as for substrates the frequent attachment of the tracer molecules to the surface is observed. For this reason, one has to distinguish between the situation where the molecules are attached to the surface and the diffusion state. The simplest approach is to neglect those steps of the movie where the determined squared displacement of the molecule is smaller than a threshold value between two frames of the movie. This was set to $0.008 \mu \mathrm{m}^{2}$ as this is about our resolution limit. When considering the mobile frames of each molecule only, one obtains average diffusion coefficients as shown in figure 6.15. A sizeable increase by a factor of two between the case of a two and a six layer film can be observed. There is also a visible movement of the molecules for the case of only the monolayer on the substrate. However, with our method of single molecule tracking this is hard to quantify. The usual procedure is to first bleach the fluorescent molecules attached to the surface. Such molecules are usually found on silicon substrates as it is impossible to clean a surface up to molecular purity. In the used setup the regions observed with the camera is smaller than the illuminated region on the sample. Therefore, the diffusing molecules have to cross a certain illuminated region in order to get into the field of view. Therefore, in order to allow the measurement of a diffusing molecule, it needs to have a minimum diffusion coefficient to reach the observed area before getting bleached. This was not the case so that only the fact, that movement of the molecules was observed, can be stated. This also is the reason why the previously mentioned "diffusion map" could not be measured.

For the quantitative comparison of the diffusion in films of different layers it is disturbing that the error bars are large compared to the case of the free-standing films. As introduced in chapter 5.1.1, the error bars show the standard deviation of the mean of all the obtained values. 


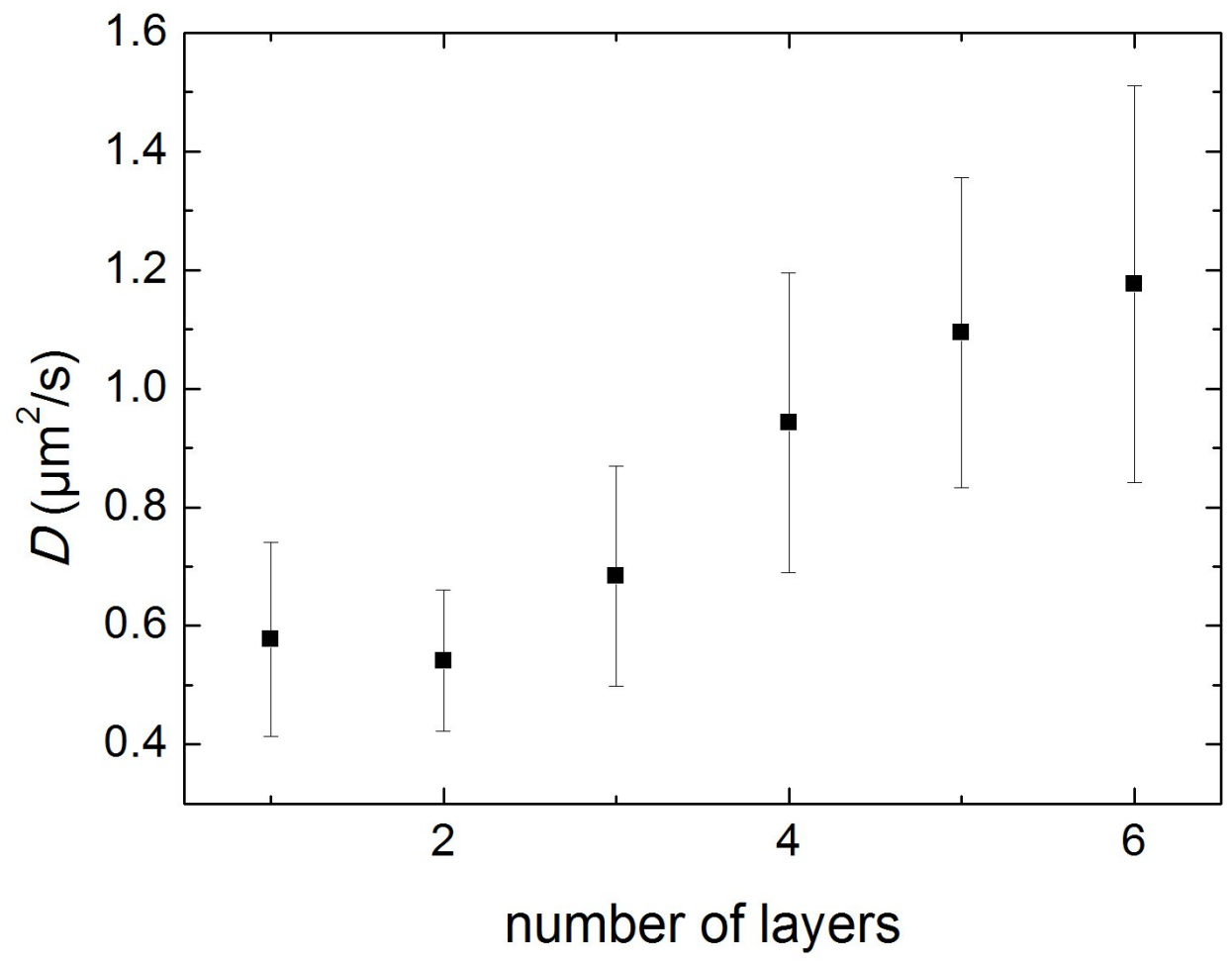

Figure 6.15: Average diffusion coefficients in thin $8 \mathrm{CB}$ films on substrates for the first six layers.

The larger spread of the diffusion coefficients in this case might be caused by binding events to the surface that are shorter than our exposure time of $20 \mathrm{~ms}$. This leads to an underestimation of the real diffusion coefficient as well as to a wider distribution. The distribution of the diffusion coefficients for the example of a five layer film is depicted in figure 6.16 .

It is interesting to note that the calculated diffusion coefficient for the film with one layer is slightly higher than the one found for the two layer film. We have seen in section 6.1.1 that the first double layer appears to be softer than the subsequent ones. This could hint to a lower density in this layer giving rise to a faster diffusion compared to the case of several layers as the free volume for each molecule is increased [59]. This might be a reason why no difference is found for the case of one or two layers. Usually one would expect a significant difference to the two layer films. The slowdown should be enhanced on further reducing the film thickness in classical theories of diffusion [148]. However, due to the big errors of those measurements, no conclusion can be made for the comparison between the films with one and two layers. 


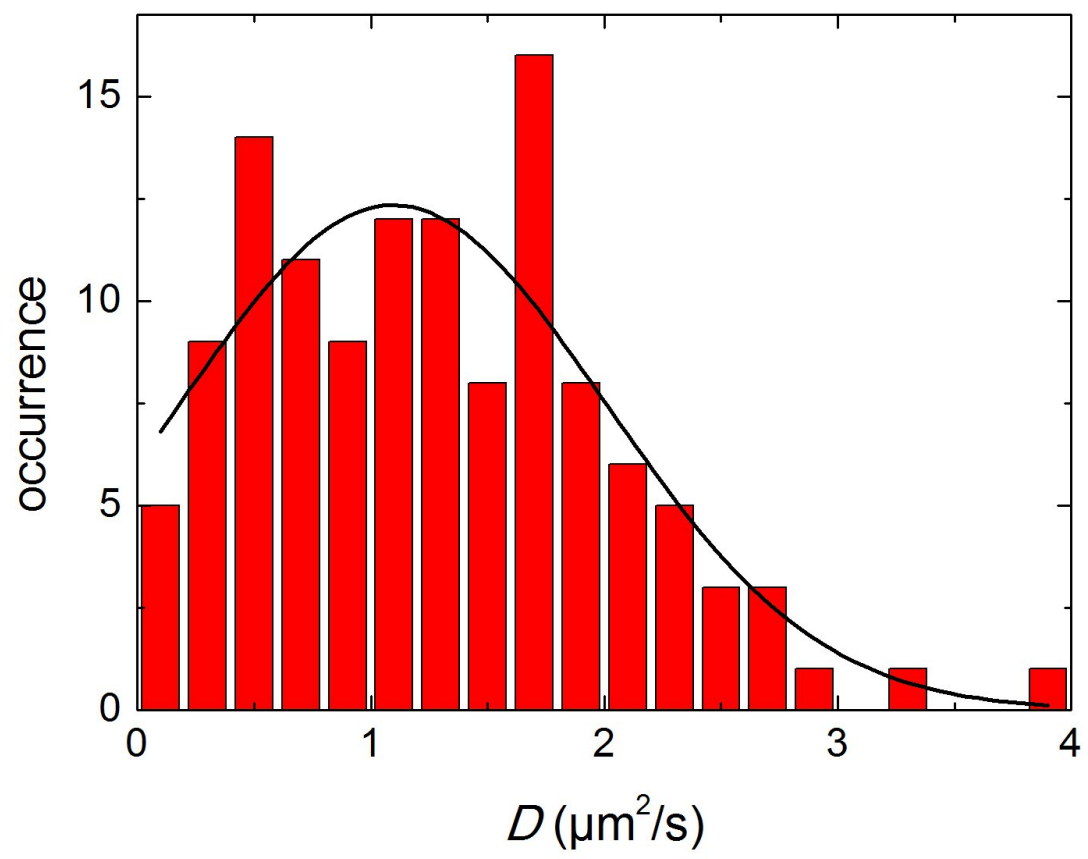

Figure 6.16: Distribution of diffusion coefficients for the example of a film consisting of five smectic layers.

\subsection{Writing of soft structures on the nanoscale}

It was shown in section 6.1.1 that it is possible to reliably prepare films with a well defined thickness using a simple technique. More interesting than this would be to selectively write and erase material from the surface. In this section the question shall be addressed, to which extent the homogeneous thin films can be used as base material for the creation of small, well-defined structures.

For this goal we use the AFM in contact mode. This mode cannot be employed to scan the inherent surfaces of soft materials as it tends to drag the material along. However, this effect can be interpreted as a feature more than as a disadvantage of contact mode scans as this makes it possible to manipulate the surface topography. The general observation when using contact mode AFM on the plane films is that the tip leaves behind a trace in the regions of scanning for all prepared films. However, there is a fundamental difference between the behaviour of films with a maximum thickness of one layer and the thicker films. Those two cases shall be treated separately in the following. 


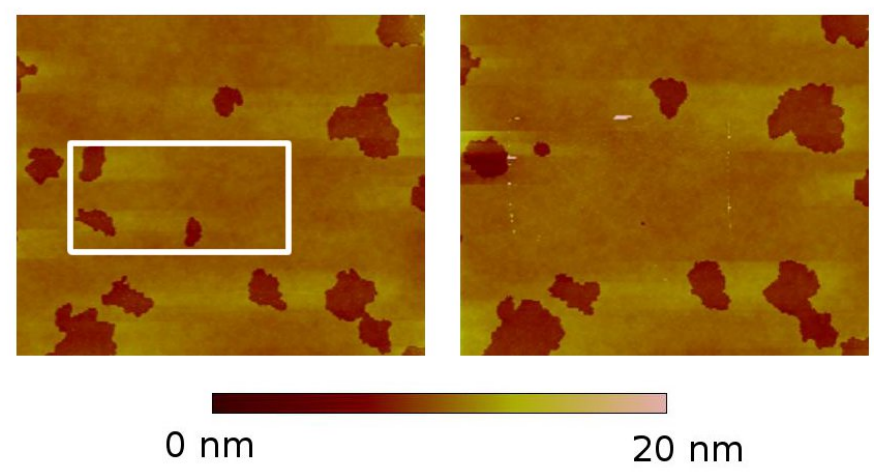

Figure 6.17: Contact mode scanning over a region of $10 \times 5 \mu \mathrm{m}^{2}$, indicated by the white rectangle on the left image. The pores in the scanned regions get filled up with material as visible on the right. The image width is 20 microns.

For the case of less than one smectic layer film thickness, one finds that contact mode AFM fills up the top layer. This means that in the scanned region one obtains a homogeneous coverage independent of the initial surface structure of the film. Also scanning over the surface several times does not change the surface topography anymore. The example where simply some of the pores in the film get filled up with material is shown in figure 6.17. A tip velocity of 2 microns per second was used for this procedure, which was a typical writing speed in our experiments.

As the material is soft and gets erased easily when the interaction force gets too large during tapping mode, it is also possible to create isolated structures by "wiping away" the material around the written structures. This is shown in figure 6.18. First a rectangle of size $10 \times 5 \mu \mathrm{m}^{2}$ is written, then one of the same size below it. Thereafter, caused by the tapping mode scan, the porous structures in the surrounding regions are erased so that finally only an isolated structure is obtained. It should be mentioned that the erasure of the structure around the written parts was unintended. When using a tip in contact mode it usually becomes unusable for the tapping mode scan. However, in this case a scan was still possible although one can see that the quality of the scans is reduced with progressing surface treatment. In most of the cases the tip needs to be replaced after the contact mode scan. It is interesting to note that the written structures appear to be more stable than the original structures despite their identical molecular structure. The reason might be that all the rounded structures of the pores are always points where an acting force is most effective. 

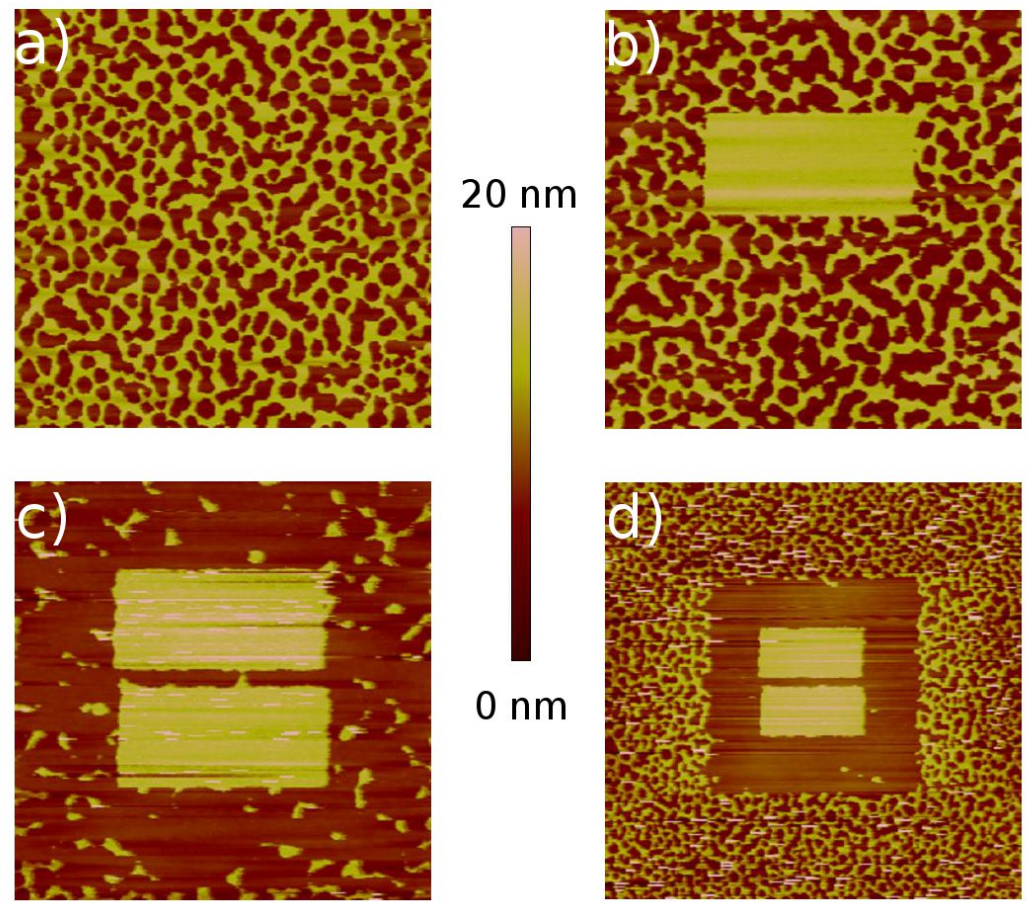

Figure 6.18: Creation of an isolated structure of one layer thickness: a) Porous structure with one smectic layer in the thicker regions after spin-coating, b) Filling of the top layer in a rectangular area of size $10 \times 5 \mu \mathrm{m}^{2}$, c) Writing a second rectangle of the same size below the original one and subsequent removal of the porous film around the structure by tapping mode, and d) Isolated structure seen in a smaller magnification. The colour scale is valid for all the images. The length scale is $20 \times 20 \mu \mathrm{m}^{2}$ for the images a) to c) and $40 \times 40 \mu \mathrm{m}^{2}$ for image d).

Generally, the removal of structure is the easier part as often this happens unwanted. In order to scan the surface without erasure, due care has to be taken to keep the interaction energy between the tip and the sample as small as possible. A certain effect is found in most of the cases, for example also for figure 6.17, where clearly the remaining pores in the film become larger.

The spatial resolution of such a scan is tested by doing a contact mode scan of only one single line which leads to a resolvable trace on the sample as shown in figure 6.19. One gets an upper bound for the line width that was produced given by the resolution of the imaging scan afterwards. The written line was determined to have a maximum width of $100 \mathrm{~nm}$. Again 

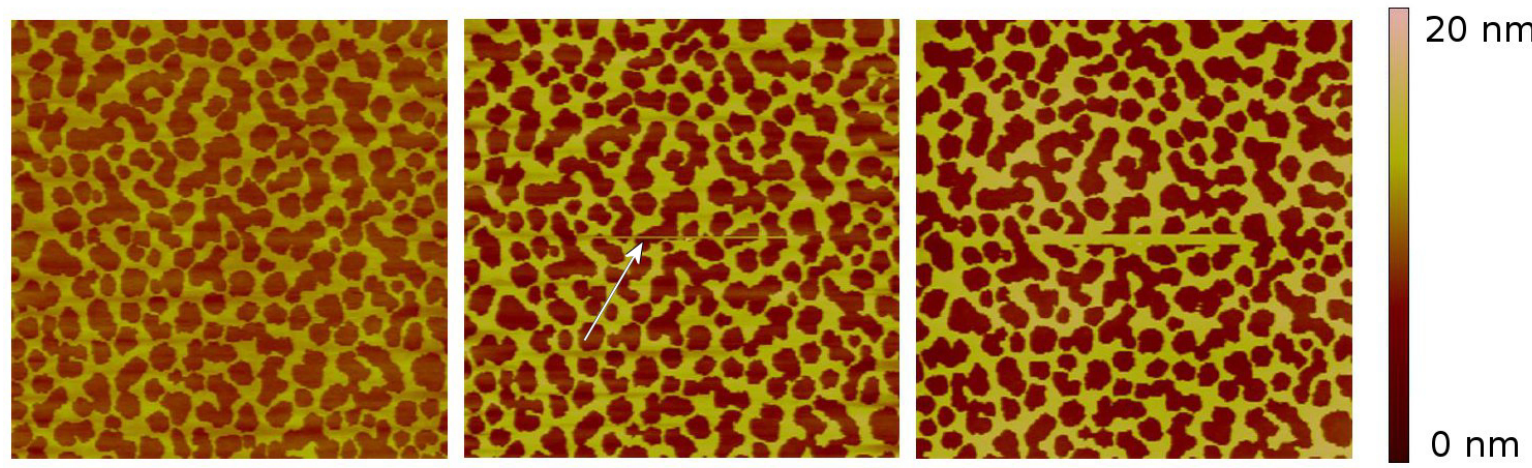

Figure 6.19: Test of the resolution of the writing process: The original surface (left) was scanned once in contact mode giving rise to a thin line (indicated by the white arrow) of around $100 \mathrm{~nm}$ width (middle). The structure can easily be extended by writing additional structures, in this case for example a bigger region of size $10 \times 0.5 \mu \mathrm{m}^{2}$ (right).

scanning over a bigger region including the same area used for the first scan only increases the written structure as also visible in figure 6.19 .

The situation is slightly different if a film consisting of more than one smectic layer is scanned in contact mode. Here, instead of just completing the topmost layer, material is added to the highest regions of the film. One finds the creation of a homogeneous film thickness regardless of the initial porous structure that was present on the film after the spin-coating. The film thickness in the scanned area is one smectic layer larger than the thickness of the higher regions of the originally prepared film. This also gives the possibility to write structures on top of each other.

However, the precision of the manipulations for the structures written on top of the existing layers appears to be smaller than the one for the filling of the first layer. An example is shown in figure 6.20 a) where a pyramidal structure was intended to be obtained (one with a huge length to height ratio, though). The regions for the scans were squares of size 10 microns, 5 microns, and 2 microns. The resulting structures appear smeared out and the original dimensions are reproduced only scarcely. Generally it is found that the edges of the structures are not as sharp as the ones with only one layer. The writing precision of $100 \mathrm{~nm}$ was not achieved for either of the higher structures. 

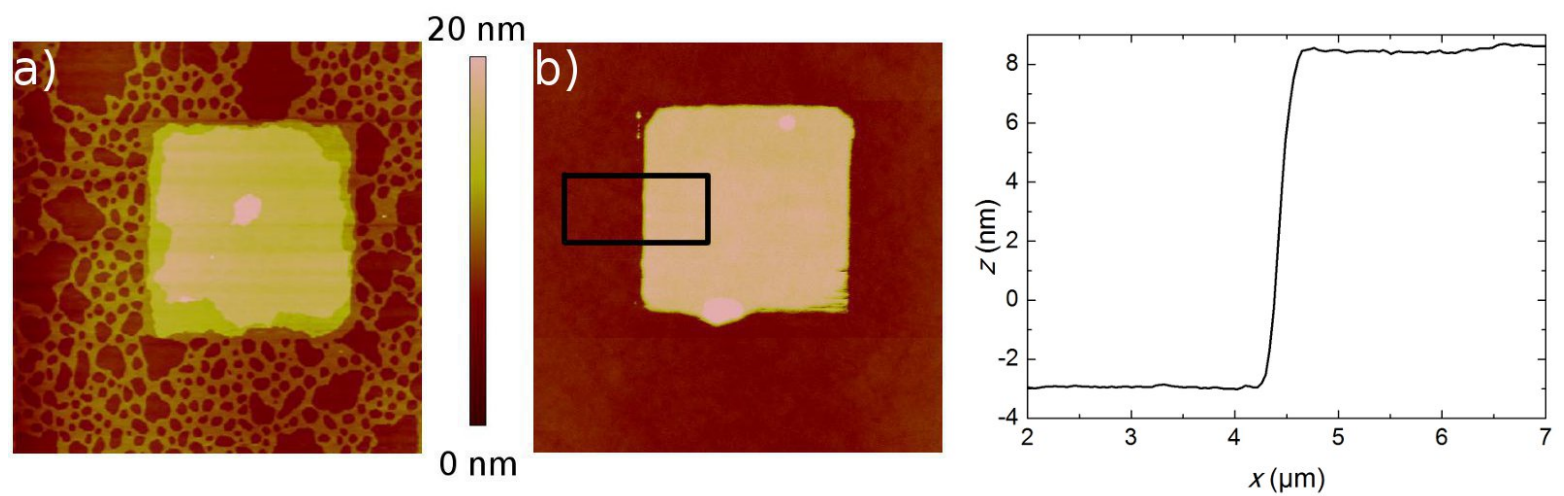

Figure 6.20: Writing of several layers on top of each other: a) Writing of three squares of size 10 microns, 5 microns, and 2 microns. b) Writing squares of edge length 10 microns leads to an isolated structure. The cross section on the right shows the step height of structure b) averaged over the region marked with the black rectangle. The total height can be estimated to be 3 layers (see text). The size of both images is $20 \times 20 \mu \mathrm{m}^{2}$.

It is also possible to write structures of identical feature size on top of each other. Subsequently structures with an arbitrary lateral height can be written. An example is shown in figure 6.20 $\mathrm{p}$ ) where twice the same region was scanned in contact mode. The structure in the surroundings disappeared and one obtains an isolated "pillar". When trying to measure the step height of the structure, a value of $11.5 \mathrm{~nm}$ is found. This value is surprising as this is not a multiple of the smectic layer spacing of $3.16 \mathrm{~nm}$. However, as already explained in section 6.1.1, the different surface properties of different regions lead to an overestimation of the real step height. Thus it can be assumed that the shown structure has a height of 3 smectic layers.

It is worthwhile mentioning that the interaction strength between tip and surface in contact mode does not play a role for the writing process (contrary to the imaging in tapping mode where the interaction strenght is crucial for proper results). Identical results were obtained when increasing the interaction by a factor of five. However, the writing process can be influenced by the writing speed of the tip. When writing faster than the mentioned two microns per second it becomes possible to also fill up the top layer for the case of thicker films in the same way as for the films with a maximum of one smectic layer. An example is shown in figure 6.21. First, a part of the big central pore was filled using a tip speed of 8 microns per second. Afterwards a rectangle with higher film thickness was written with the usual speed to show that is was not an artifact caused by specific features of the tip. 

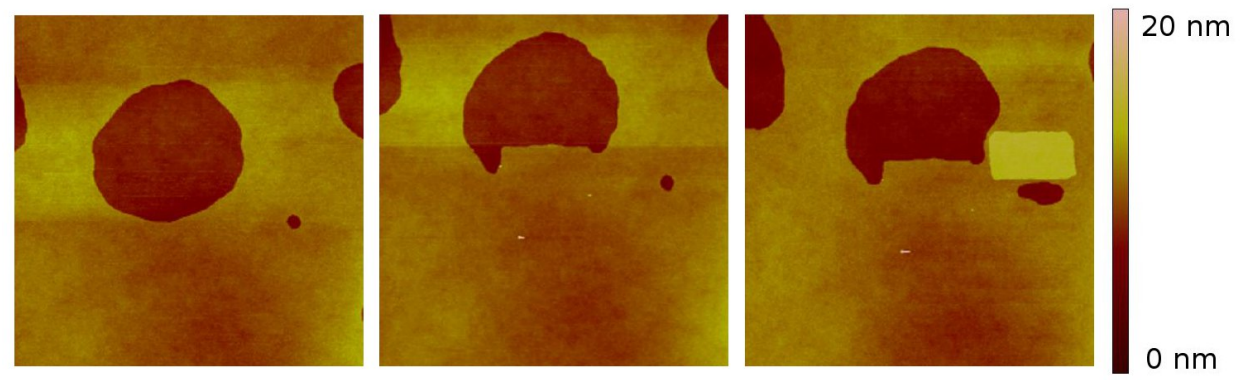

Figure 6.21: After the preparation of a film with a thickness of three layers in the higher areas, two kinds of processes are shown: From left to the middle the filling of the pores in the lower region of the central hole. Secondly, from the middle to the right a rectangle of size $10 \times 5 \mu \mathrm{m}^{2}$ was written. The image size is $40 \times 40 \mu \mathrm{m}^{2}$.

Using the properties of the writing described in the last paragraph, we can suggest the physical process responsible for the writing. The fact that the writing procedure does not seem to depend on the interaction strength of the tip with the surface suggests that there is no molecular force that is causing the attraction of the additional material. A possible explanation would be that there is a permanent meniscus between the tip and the liquid crystal film which in case of one layer fills the holes in the films and which in case of more than one layer leaves behind a trace in form of an additional molecular layer as depicted in figure 6.22. This concept is closely related to the mechanism of dip-pen nanolithography (DPN) [149]. However, in our samples the material that is deposited on the film is directly supplied by the film itself and does not come as "ink" loaded on the AFM tip. As our samples combine the surface stabilization of the structures with the mobility on molecular level, a material transport to the place of writing is possible as we are working with low tip speeds. This means that the deposited material is not necessarily removed from the direct proximity but taken from the film as a whole (an example can be seen in figure 6.20a), where substantial material was added to the central region but no material disappeared from the surroundings). Thus, comparing the size of the entire film being in the range of centimeters with the micrometer size of the written structures, a huge reservoir of "ink" is available. This explanation by a meniscus is supported by the finding that in case of a 4 times faster scanning there is no additional layer added in the thicker films but only the gaps are filled in case of a film which has pores as it has been shown in figure 6.21. When the tip is moved faster, the meniscus would be smaller for which reason the trace of the tip is smaller in height and only the filling up of the topmost layer is observed. Also the different 


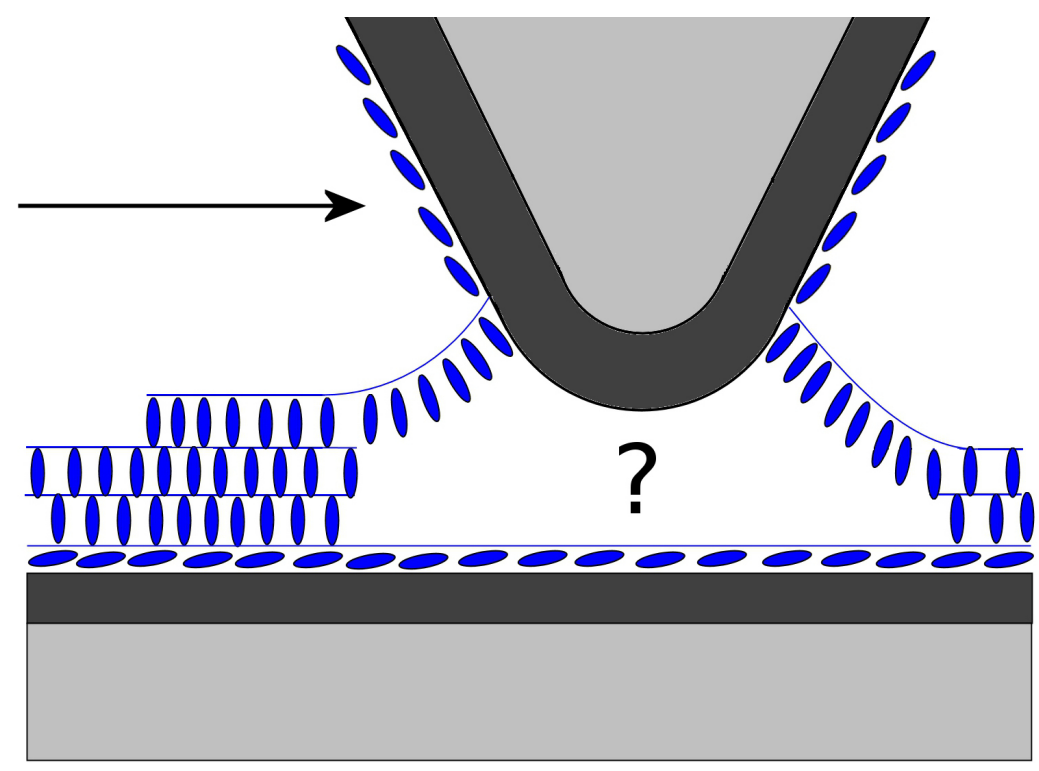

Figure 6.22: Sketch for the suggested mechanism of writing a new molecular layer: In contact mode a permanent meniscus between the tip (diameter of $9 \mathrm{~nm}$ according to the supplier specifications) and the liquid crystal is formed. This leaves behind a trace in form of a films with a thickness of one molecular layer. The arrow gives the direction of scanning. The molecular structure directly below the tip is unclear and most probably full of defects due to contradictory constraints from all sides. light grey: silicon, dark grey: native silicon oxide.

behaviour of the film with one layer is understandable in this sense as the smaller availability of material might lead to a smaller meniscus that is not creating an additional layer.

It shall now be considered if it is realistic that simply by diffusion enough material for the writing can be transported to the spot where the tip meets the film. For this purpose, a rough estimate will be done on how big the flow of material has to be and if this matches with the diffusion coefficients measured in section 6.3 . When writing one layer on top of the existing layers with the standard procedure, it took around 45 minutes to write a larger squared structure of size $10 \times 10 \mu \mathrm{m}^{2}$. As the height of the layer is around $3 \mathrm{~nm}$, the necessary material transport in the direction of the writing spot has to be of the order of $10^{-22} \mathrm{~m}^{3} / \mathrm{s}$. Using the molar volume of the $8 \mathrm{CB}$ molecules of $0.29 \mathrm{l} / \mathrm{mol}$, 1 one obtains a necessary a molar flux of $3 \cdot 10^{-19} \mathrm{~mol} / \mathrm{s}$. This material has to have entered into the written region through the cross

\footnotetext{
${ }^{1}$ The molar volume was determined using the software ACD/ChemSketch Version 12.01.
} 
section of the square. As the writing of new structures only takes place at a minimum film thickness of two layers, it has to enter through a cross section of at least $6 \mathrm{~nm} \times 40 \mu \mathrm{m}$. Thus, the diffusion flux is of the order of

$$
j=10^{-6} \frac{\mathrm{mol}}{\mathrm{m}^{2} \mathrm{~s}} .
$$

This value can be used for Fick's law from equation 3.1 together with the diffusion coefficient of around $10^{-12} \mathrm{~m}^{2} / \mathrm{s}$ measured in section 6.3 . The obtained radial concentration gradient is around

$$
\frac{\partial c}{\partial r}=\left|\frac{j}{D}\right|=10^{6} \frac{\mathrm{mol} / \mathrm{m}^{3}}{\mathrm{~m}}
$$

at the border of the written square. Approximating the directed motion as being directed towards the center of the written region, the gradient has to decrease inversely proportional to the distance $r$ from this center:

$$
\frac{\partial c}{\partial r}=\frac{A}{r}
$$

The constant $A$ is found with the known value at $r=5 \mu \mathrm{m}$ from equation 6.2 as $A=5 \frac{\mathrm{mol}}{\mathrm{m}^{3}}$. The total difference in the concentration is finally found as the integral from the edge of the written structure $\left(r_{1}=5 \mu \mathrm{m}\right)$ to the end of the zone of influence from where the material would be taken away. This shall be assumed to be $r_{2}=1 \mathrm{~mm}$ somewhat arbitrarily. The obtained difference in concentration is

$$
\Delta c=A \cdot \ln \frac{r_{2}}{r_{1}}=26 \frac{\mathrm{mol}}{\mathrm{m}^{3}} .
$$

This can be compared to the equilibrium concentration (which is the inverse of the mentioned molar volume) of $c_{0}=3450 \mathrm{~mol} / \mathrm{m}^{3}$. One finds that the change in concentration is around $0.8 \%$. A density change of this order would also be obtained for a change in temperature of $7 \mathrm{~K}$ within the smectic phase [80]. One can thus conclude that the material transport necessary for supplying enough liquid crystal for the new structure can be explained by diffusional transport inside the film under the assumption of a reasonable concentration gradient.

The general physical process seems to be understood. In most of the cases the behaviour consistently shows exactly the properties that were described in this section. However, occasionally a deviating behaviour is found. This suggests that the procedure is not completely robust and apparently has a critical dependence on the features of the tip. The shape of a real AFM tip is rarely an ideally rounded object as it is depicted in figure 6.22. Such changes might have a strong impact on the processes involved in the formation of the surface structures. As a consequence, it is sometimes found that for example more than one layer is written at once as shown in figure 6.23 a). This can also be considered as a possibility to write higher structures 
a)
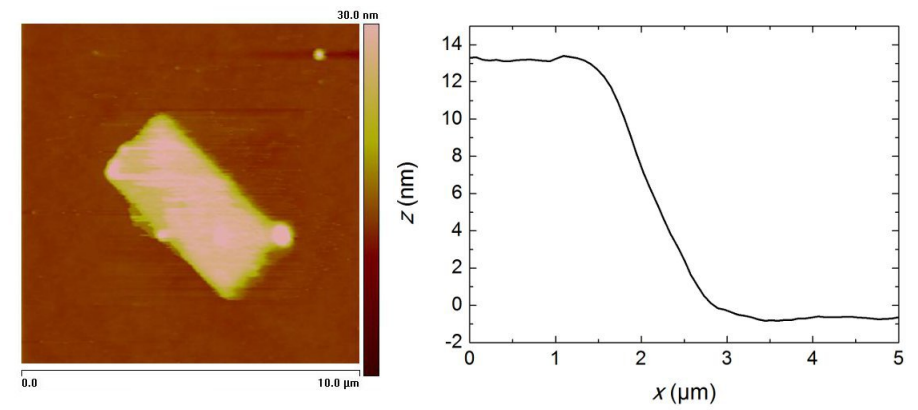

b)
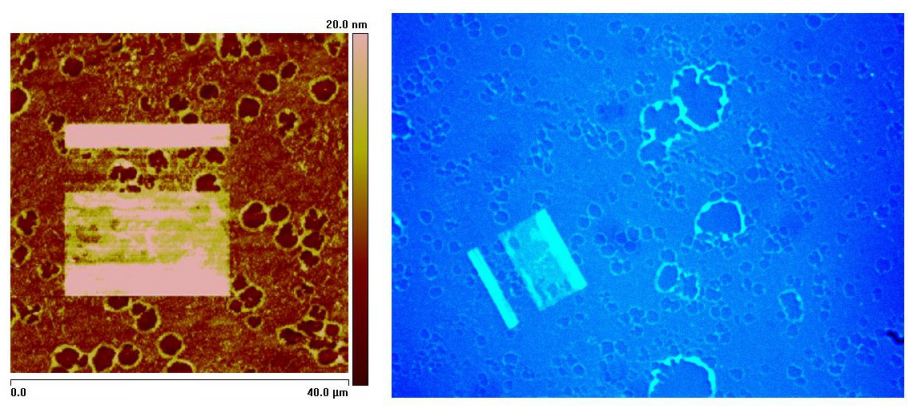

Figure 6.23: Examples for more than one smectic layer written during one scan: a) one contact mode scan leads to a feature height of 4 smectic layers, b) Writing in tapping mode with a broken tip creates a film with around 5 layers of maximum thickness that does not appear dense. This effect can also be seen optically (for the optical image the written structure gives the scale with its edge length of 20 microns).

in a shorter time if it is possible to control the tip properties more precisely.

For another sample it was found that scanning a surface in tapping mode using a broken tip also leaft behind a structure as shown in figure 6.23 $\mathrm{b}$ ). This can probably be assigned to a temporary meniscus of liquid crystal material between the tip and the film. Hence, when trying to actually image the surface a structure was found to be written in a similar way as when using the contact mode. As this effect was found for the case of structures on a silicon substrate with $100 \mathrm{~nm}$ oxide, it was also possible to image the structures optically as in this case the contrast is strongly enhanced compared to substrates with native oxide [150]. During this writing in tapping mode the scan parameters were varied. One can see that in this case the interaction parameters of the tip play an important role as partially the original structure of the porous film is still visible both in the AFM image and in the optical image. This means that the higher regions do not form a complete film in this case but create a thickness fluctuation on the scale of one hundred nanometers. No change in these tiny structures was observed 

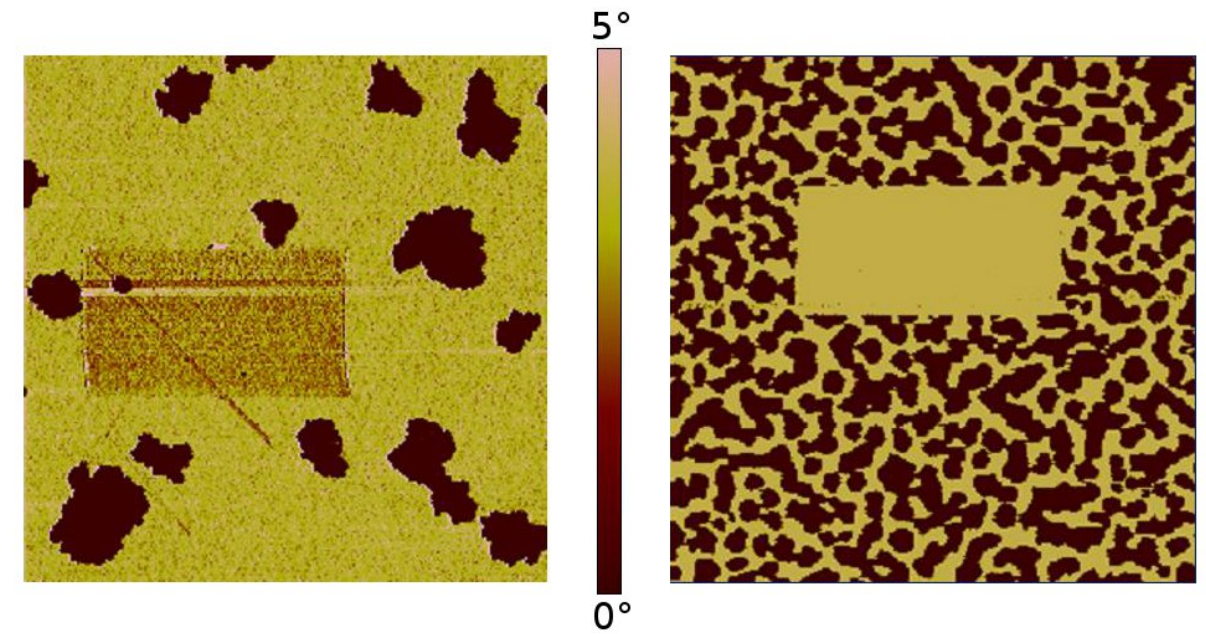

Figure 6.24: Variable phase properties of the first written layer. The left image shows the phase image of figure 6.17 and the right image the phase image of figure 6.18b). In both cases the structure height is identical with the the one of the surrounding structures. However, the left image shows a phase contrast while the right one does not.

over hours. Hence, using appropriate parameters one can create surface structures that are not dense but patterned on a small scale. The tip velocity for the described experiment was 8 microns per second. Thus, in this case a structure that can be estimated to have a thickness of 5 smectic layers has been written with a 4 times faster writing speed compared to the normal protocol for the contact mode. These observations show that the tip properties play a major role in the formation of the written surface structures. It is obvious that this applies to both the tip material and the tip geometry.

An open question is, in which way the surface properties can be changed by the contact mode scan. It is sometimes found that despite having an identical layer height, the surface properties differ. Figure 6.24 shows two phase images of the completion of the first layer by contact mode scanning. However, in the right of the two images there is no difference between the regions that from the beginning had a thickness of one smectic layer and the scanned region, whereas in the left image the rectangular region is very well visible. It is not obvious what can cause the difference in the surface properties revealed by the contrast in the phase image. As the orientation is defined by the alignment at the air interface this cannot differ for the two regions. Also the identical film thickness can only be achieved by an identical internal structure. As in a molecularly mobile system the density also should not differ in 
the equilibrium state, there are no obvious candidates for an explanation of the difference in surface properties. Of course it would be desirable to first find out the decisive parameter that changes the surface properties and then to controllably generate different properties in different regions.

In summary it was shown that contact mode AFM can be used to manipulate the film thickness with an axial resolution of one smectic layer. The lateral resolution is dependent on the height of the original film and is in best case below $100 \mathrm{~nm}$. The inherent mobility in the film is able to supply the necessary material in order to transport enough "ink" to the spot of writing, which is a key advantage compared to other methods of soft matter writing [149]. Also the experiments suggest that the tip is a main control parameter for the writing of the structure. Further experiments will have to reveal to which extent the use of different tips can still improve different writing parameters like the resolution, the writing speed, or the size of the structures written in one contact mode scan. 


\section{Chapter 7}

\section{Summary and outlook}

The work presented in this thesis studies a variety of phenomena observable in thin films of smectic liquid crystals. For the two different cases of the presence or the absence of an underlying substrate, the dynamic and structural properties of the films were addressed. For the films without substrate (free-standing films), the diffusional behaviour was analysed concerning the two aspects of strong geometrical confinement and behaviour in the vicinity of different kinds of phase transitions. The used method was single molecule tracking meaning that dye molecules are dissolved in a very dilute concentration which makes it possible to follow the movement of individual molecules. The films on substrates were studied with respect to the structures formed on the surface. First, it was observed how the material self-organises after spin-coating from a toluene solution. Then, it was studied how the surface structure can be influenced using atomic force microscopy in contact mode.

In the first part of the thesis, the focus was put on free-standing film with a thickness of only few molecular layers. The inherent translational molecular dynamics was studied. The particularity of those films arises from the absence of a supporting substrate. Therefore, compared to other studies on thin films, the dynamics does not mainly represent the influence of a solid interface but the one of the free surface. In the smectic A phase, a considerably faster diffusion was observed when the film thickness was decreased down to two smectic layers. The general behaviour and the functional dependence appears to be similar for all different compounds regardless of the molecular properties. However, the magnitude of the effect varies with the material. Depending on the mesogen, the difference between the extrapolated bulk diffusion coefficient and the value for two layers is ranging from a factor of 2.5 to a factor of 5. This effect can not be explained by the classical argument of a faster diffusion at the 
free surface caused by the low friction. The results were confirmed by molecular dynamics simulation concerning the functional dependence. Still the small data base of simulations existing so far restricts the possibilities for quantitative comparisons. In the future, simulations will be able to show if the absolute changes of the diffusion coefficient can be correlated with molecular properties as for example the aspect ratio of the molecule or its dipole moment.

For free-standing films that are close to phase transitions, the temperature dependence of the diffusion turned out to vary with the respective type of phase transition. First, staying within the smectic A phase, the example of the mesogen $8 \mathrm{CB}$ was studied. There was no sign of any impact of the geometrical confinement on the diffusion. The temperature dependence of the diffusion coefficient was found to obey the classical Arrhenius behaviour. This is in agreement with the behaviour observed for the bulk material. Contrary to that, for materials with a transition to a phase with in-plane ordering, the functional dependence was shown to resemble a glass transition. In this respect, the two examples of a transition from smectic $\mathrm{C}$ to smectic $\mathrm{G}$ and from smectic $\mathrm{A}$ to smectic $\mathrm{F}$ showed a similar behaviour. For the transition from the smectic $\mathrm{A}$ to smectic $\mathrm{C}$ phase, there is a visible difference between a first order and a second order transition. In case of a second order transition, the temperature dependence is monotonous and smooth. In case the transition is first order, a dip in the diffusion coefficient in the vicinity of the phase transition was observed. This slowdown of the diffusion can be explained by the density fluctuations around the phase transition that also give rise to the first order character of the phase transition.

It was found that the scaling of the diffusion with temperature does not change with changing film thickness. The properties observed for one material for a certain film thickness were found for all the other film thicknesses as well. This is not a trivial statement as it was already mentioned that the film thickness strongly influences the absolute value of the diffusion. The unknown mechanisms responsible for this could have as well changed the temperature dependence. At least for the material presented here, this is not the case.

However, owing to the limited number of compounds studied in this work, it was by far not possible to give a full classification of the behaviour at the different phase transitions in free-standing films. Partially this is also due to the lack of available materials as for example it is a rare case that the transition from smectic $\mathrm{A}$ to smectic $\mathrm{C}$ is first order. Also, the large number of possible smectic phases - that all can be stabilised in free-standing films - makes it difficult to give a generalised picture of the behaviour at phase transitions.

In some cases, spatially inhomogeneous films possessing phase boundaries could be prepared. For these films, it was shown that the molecules have a strong tendency to stay in their 
phase. This was observed both for the case of laterally and axially inhomogeneous films. Particularly for the case of axially inhomogeneous films (meaning different smectic layers are in different phases), the movement at the molecular level turned out to be substantially confined. Molecules were found to travel tens of micrometres in the plane of the smectic layers without moving even ten nanometres perpendicular to it. This difference is by far larger than the normal anisotropy in the diffusion of smectic liquid crystals where the difference in the diffusion coefficient is usually not larger than a factor of two.

The second part of the thesis is concerned with smectic films on silicon substrates. It was shown that the creation of film thicknesses with a precision far better than one smectic layer can be achieved using a simple spin-coating technique. The inner structure of the film could be concluded with the help of ellipsometry measurements as being a planar monolayer on the substrate surface followed by homeotropic smectic layers. The smectic layer at the air interface is usually found to be incomplete. Only for discrete values of the concentration used for the spin-coating, a full coverage was found. Structures that were found on the surface are isolated island for a small coverage of the top layer; for larger coverages starting from $35 \%$, the observed suface structures connect to a two dimensional porous structure. The structures of the incomplete top layer appeared to be independent of the amount of underlying material for a small number of total layers. The feature size of both observed structures is of the order of one micron.

The films were found to remain in the smectic phase above the bulk phase transition to the nematic phase. As for the nematic phase a dewetting was observed, the temperature of dewetting could be used to estimate the change in the phase transition temperature. The droplets that form in the nematic phase also offer another way to quantify the surface induced formation of smectic layers more precisely. When they are cooled to temperatures slightly above the phase transition to the smectic phase again, the formation of smectic steps at the edge of the drops is observed. The number of layers observable depending on the temperature showed the layer-by-layer transition of the material close to the substrate surface. With the help of this method, up to seven smectic layers could be identified in the presence of a nematic main part of the droplet.

It is astonishing that all the created structures turned out to be stable for days despite the mobility at molecular level. The measurement of the diffusion in those films showed a slowdown in the diffusion by a factor of two when going from six smectic layers to one 
smectic layer. This change alone cannot explain the structural stability. It is evident that the presence of the solid interface stabilises the structure but the physical process behind this is not clear. Because of the limited number of molecules involved in the process, this system might also be a good candidate for molecular dynamics simulations. This could give more insight into the necessary interactions between liquid crystal and substrate that render the structures stable.

It was shown that the thin films can be manipulated on the nanoscale. Using the standard contact mode AFM, it is possible to write structures onto the existing film. It was shown that for only one layer thickness the writing results in the filling of the top layer. The lateral writing precision is better than $100 \mathrm{~nm}$. For larger film thicknesses, there are structures added on top of the existing ones. Here, the precision in the height of the written layers is given by the molecular layer spacing, which means around 3 nanometers. The physical mechanism behind the writing is similar to the established technique of dip-pen nanolithography. It also has the property that the interaction strength during the contact mode scan does not influence the written structure. This is one of the main criteria for a possible parallelisation of the described procedure.

For all kinds of manipulations using AFM, the exact properties of the AFM tip influence the properties of the writing process. This is also the case for our method. The possibility to increase the writing speed and the amount of deposited material were found to be attainable. Also, the writing of a non-dense layer with a film thickness varying on length scales in the order of less than hundred nanometers was achieved. However, a lot of work is still to be done to create such structures in a controllable way. The shape of the the tip as well as the tip material is expected to have a strong impact on the process of writing. Further studies in this direction seem indispensable in order to fully understand this effect that can be seen as an interesting step in the direction of a flexible tool for soft matter nanolithography. 


\section{Appendix}

\section{Binarisation of AFM images}

For the binarisation of the AFM images of the film on silicon substrates the software MATLAB was using. The used code is as follows:

$\operatorname{Img}=$ imread('***input_file***');

$\operatorname{Img} 2=\operatorname{Img}(25: 386,7: 368,1)$;

grauwert $=$ graythresh $(\operatorname{Img} 2)$;

Imgbw=im2bw(Img2, grauwert);

imshow(Imgbw);

$\mathrm{w}=\operatorname{sum}(\operatorname{sum}(\operatorname{Imgbw}))$;

totalpixels $=\operatorname{size}(\operatorname{Imgbw}, 1) * \operatorname{size}(\operatorname{Imgbw}, 2)$;

$\operatorname{disp}($ num2str(w/totalpixels));

Ispeichern $=$ getframe $(\mathrm{gcf})$;

imwrite(Ispeichern.cdata, '***output_file***')); 



\section{References}

[1] H. Schüring and R. Stannarius, "Surfaces and interfaces of free-standing smectic films," Molecules in interactions with surfaces and interfaces. Lecture notes on physics, vol. 634, pp. 337-381, 2004.

[2] B. Jérôme, "Surface effects and anchoring in liquid crystals," Reports on Progress in Physics, vol. 54, pp. 391-451, 1991.

[3] L. Zou, J. Wang, P. Basnet, and E. K. Mann, "Line tension and structure of smectic liquid-crystal multilayers at the air-water interface," Physical Review E, vol. 76, p. 031602(6), 2007.

[4] R. Garcia, E. Subashi, and M. Fukuto, "Thin-thick coexistence behavior of 8CB liquid crystalline films on silicon," Physical Review Letters, vol. 100, p. 197801(4), 2008.

[5] W. Guo and C. Bahr, "Influence of anchoring strength on focal conic domains in smectic films," Physical Review E, vol. 79, p. 011707(5), 2009.

[6] J.-P. Michel, E. Lacaze, M. Goldmann, M. Gailhanou, M. de Boissieu, and M. Alba, "Structure of smectic defect cores: X-ray study of 8CB liquid crystal ultrathin films," Physical Review Letters, vol. 96, p. 027803(4), 2006.

[7] A. V. Kachynski, A. N. Kuzmin, P. N. Prasad, and I. I. Smalyukh, "Coherent antiStokes Raman scattering polarized microscopy of three-dimensional director structures in liquid crystals," Applied Physics Letters, vol. 91, p. 151905(3), 2007. 
[8] I. Smalyukh, R. Pratibha, N. Madhusudana, and O. Lavrentovich, "Selective imaging of 3D director fields and study of defects in biaxial smectic A liquid crystals," The European Physical Journal, vol. 16, pp. 179-191, 2005.

[9] D. K. Yoon, M. C. Choi, Y. H. Kim, M. W. Kim, O. D. Lavrentovich, and H.-T. Jung, "Internal structure visualization and lithographic use of periodic toroidal holes in liquid crystals," Nature Materials, vol. 6, pp. 866-870, 2007.

[10] Y. H. Kim, D. K. Yoon, H. S. Jeong, J. H. Kim, E. K. Yoon, and H.-T. Jung, "Fabrication of a superhydrophobic surface from a smectic liquid-crystal defect array," Advanced Functional Materials, vol. 19, pp. 3008-3013, 2009.

[11] H. Ringsdorf, B. Schlarb, and J. Venzmer, "Molekulare Architektur und Funktion von polymeren orientierten Systemen - Modelle für das Studium von Organisation, Oberflächenerkennung und Dynamik bei Biomembranen," Angewandte Chemie, vol. 100, pp. 117-162, 1988.

[12] S. Chandrasekhar and G. S. Ranganath, "Discotic liquid crystals," Reports on Progress in Physics, vol. 53, pp. 57-84, 1990.

[13] M. Lehmann, S.-W. Kang, C. Köhn, S. Haseloh, U. Kolb, D. Schollmeyer, Q. B. Wang, and S. Kumar, "Shape-persistent V-shaped mesogens - formation of nematic phases with biaxial order," Journal of Materials Chemistry, vol. 16, pp. 4326-4334, 2006.

[14] G. W. Gray and J. W. Goodby, Smectic Liquid Crystals. Leonard Hill, 1984.

[15] P. G. de Gennes and J. Prost, The Physics of Liquid Crystals. Oxford University Press, 1993.

[16] R. Pindak, D. E. Moncton, S. C. Davey, and J. W. Goodby, "X-ray observation of a stacked hexatic liquid-crystal B phase," Physical Review Letters, vol. 46, pp. 1135$1138,1981$.

[17] C.-Y. Chao, C.-F. Chou, J. T. Ho, S. W. Hui, A. Jin, and C. C. Huang, "Nature of layer-by-layer freezing in free-standing 40.8 films," Physical Review Letters, vol. 77, pp. 2750-2753, 1996. 
[18] C. Bahr, T. Dorfmüller, H. Haberland, G. Heppke, S. Hess, H. Jockusch, K. Lüders, J. Seidel, R. Thull, H. Tschesche, and B. Wende, Vielteilchen-Systeme. Walter de Gruyter, 1992.

[19] S. Ishihara, "How far has the molecular alignment of liquid crystals been elucidated?," Journal of Display Technology, vol. 1, pp. 30-40, 2005.

[20] S. Chandrasekhar, Liquid Crystals. Cambridge University Press, 1992.

[21] M. Kléman and O. D. Lavrentovich, Soft Matter Physics: An Introduction. SpringerVerlag, 2003.

[22] W. Maier and A. Saupe, "Eine einfache molekulare Theorie des nematischen kristallinflüssigen Zustandes," Zeitschrift für Naturforschung, vol. 13a, pp. 564-566, 1958.

[23] W. Maier and A. Saupe, "Eine einfache molekular-statistische Theorie der nematischen kristallinflüssigen Phase. Teil i,” Zeitschrift für Naturforschung, vol. 14a, p. 882, 1959.

[24] W. Maier and A. Saupe, "Eine einfache molekular-statistische Theorie der nematischen kristallinflüssigen Phase. Teil ii,” Zeitschrift für Naturforschung, vol. 15a, p. 287, 1960.

[25] G. R. Luckhurst and C. Zannoni, "Why is the Maier-Saupe theory of nematic liquid crystals so successful?," Nature, vol. 267, pp. 412-414, 1977.

[26] P. G. de Gennes, "An analogy between superconductors and smectics A," Solid State Communications, vol. 11, pp. 753-756, 1972.

[27] P. G. de Gennes, "Sur la transition smectique A - smectique C," Comptes Rendus, Hebdomadaires des Seances de l'Academie des Sciences, Serie B, vol. 274, pp. 758$760,1972$.

[28] G. Friedel and F. Grandjean, "Observation geometriques sur les liquidesa coniques focales," Bulletin de la Société Française de Minéralogie et de Crystallographie, vol. 33, p. 409, 1910.

[29] J. B. Fournier, I. Dozov, and G. Durand, "Surface frustration and texture instability in smectic-A liquid crystals," Physical Review A, vol. 4, pp. 2252-2255, 1990. 
[30] V. Designolle, S. Herminghaus, T. Pfohl, and C. Bahr, "AFM study of defect-induced depressions of the smectic-A/air interface," Langmuir, vol. 22, pp. 363-368, 2006.

[31] B. Zappone, E. Lacaze, H. Hayeb, M. Goldmann, N. Boudet, P. Barois, and M. Alba, "Self-ordered arrays of linear defects and virtual singularities in thin smectic-A films," Soft Matter, vol. 7, pp. 1161-1167, 2011.

[32] J.-P. Michel, E. Lacaze, M. Alba, M. de Boissieu, M. Gailhanou, and M. Goldmann, "Optical gratings formed in thin smectic films frustrated on a single crystalline substrate," Physical Review E, vol. 70, p. 011709(12), 2004.

[33] M. P. Valignat, S. Villette, J. Li, R. Barberi, R. Bartolino, E. Dubois-Violette, and A. M. Cazabat, "Wetting and anchoring of a nematic liquid crystal on a rough surface," Physical Review Letters, vol. 77, pp. 1994-1997, 1996.

[34] I. Drevensek Olenik, K. Kocevar, I. Musevic, and T. Rasing, "Structure and polarity of 8CB films evaporated onto solid substrates," The European Physical Journal E, vol. 11, p. 169-175, 2003.

[35] O. Bénichou, M. Cachile, A. M. Cazabat, C. Poulard, M. P. Valignat, F. Vandenbrouck, and D. V. Effenterre, "Thin films in wetting and spreading," Advances in Colloid and Interface Science, vol. 100-102, pp. 381-398, 2003.

[36] S. Bardon, R. Ober, M. P. Valignat, F. Vandenbrouck, A. M. Cazabat, and J. Daillant, "Organization of cyanobiphenyl liquid crystal molecules in prewetting films spreading on silicon wafers," Physical Review E, vol. 59, pp. 6808-6818, 1999.

[37] L. Xu, M. Salmeron, and S. Bardon, "Wetting and molecular orientation of 8CB on silicon substrates," Physical Review Letters, vol. 84, pp. 1519-1522, 2000.

[38] J. Xue, C. S. Jung, and M. W. Kim, "Phase transitions of liquid-crystal films on an air-water interface," Physical Review Letters, vol. 69, pp. 474-477, 1992.

[39] P. Pieranski, L. Beliard, J.-P. Tournellec, X. Leoncini, C. Furtlehner, H. Dumoulin, E. Riou, B. Jouvin, J.-P. Fknerol, P. Palaric, J. Heuving, B. Cartier, and I. Kraus, "Physics of smectic membranes," Physica A, vol. 194, pp. 364-389, 1993.

[40] W. H. de Jeu, B. I. Ostrovskii, and A. N. Shalaginov, "Structure and fluctuations of smectic membranes," Reviews of Modern Physics, vol. 75, pp. 181-235, 2003. 
[41] J.-C. Géminard, R. Holyst, and P. Oswald, "Meniscus and dislocations in free-standing films of smectic-A liquid crystals," Physical Review Letters, vol. 78, pp. 1924-1927, 1997.

[42] F. Picano, P. Oswald, and E. Kats, "Disjoining pressure and thinning transitions in smectic-A liquid crystal films," Physical Review E, vol. 63, p. 021705(9), 2001.

[43] J. Bechhoefer, J.-C. Géminard, L. Bocquet, and P. Oswald, "Experiments on tracer diffusion in thin free-standing liquid-crystal films," Physical Review Letters, vol. 79, pp. 4922-4925, 1997.

[44] F. Heslot, N. Fraysse, and A. M. Cazabat, "Molecular layering in the spreading of wetting liquid drops," Nature, vol. 338, pp. 640-642, 1989.

[45] M. L. Forcada and M. Mate, "Molecular layering during evaporation of ultrathin liquid films," Nature, vol. 363, pp. 527-529, 1993.

[46] C.-J. Yu, A. G. Richter, A. Datta, M. K. Durbin, and P. Dutta, "Observation of molecular layering in thin liquid films using X-ray reflectivity," Physical Review Letters, vol. 82, pp. 2326-2329, 1999.

[47] P. S. Pershan, A. Braslau, A. H. Weiss, and J. Als-Nielsen, "Smectic layering at the free surface of liquid crystals in the nematic phase: X-ray reflectivity," Physical Review A, vol. 35, pp. 4800-4813, 1987.

[48] R. Lucht, C. Bahr, and G. Heppke, "Layering transitions at the free surface of a smectic liquid crystal," Journal of Physical Chemistry B, vol. 102, pp. 6861-6864, 1998.

[49] S. Heinekamp, R. A. Pelcovits, E. Fontes, E. Y. Chen, R. Pindak, and R. B. Meyer, "Smectic-C* to smectic-A transition in variable-thickness liquid-crystal films: Orderparameter measurements and theory," Physical Review Letters, vol. 52, pp. 1017-1020, 1984.

[50] D. Schlauf, C. Bahr, M. Glogarova, M. Kaspar, and V. Hamplova, "Temperature difference between bulk and surface transition in freely suspended smectic films," Physical Review E, vol. 59, pp. 6188-6191, 1999. 
[51] A. J. Jin, T. Stoebe, and C. C. Huang, "Nature of the layer-by-layer transition associated with the smectic-A-crystal-B transition in free-standing liquid-crystal films," Physical Review E, vol. 49, pp. R4791-R4794, 1994.

[52] T. Stoebe and C. C. Huang, "Physical properties of thin substrate-free liquid-crystal films," International Journal of Modern Physics B, vol. 9, pp. 2285-2319, 1995.

[53] R. Geer, T. Stoebe, C. C. Huang, R. Pindak, G. Srajer, J. W. Goodby, M. Cheng, J. T. Ho, and S. W. Hui, "Hexatic and crystal phase transitions in thin free-standing liquidcrystal films," Physical Review Letters, vol. 66, pp. 1322-1325, 1991.

[54] R. Geer, T. Stoebe, C. C. Huang, R. Pindak, J. W. Goodby, M. Cheng, J. T. Ho, and S. W. Hui, "Liquid-hexatic phase transitions in single molecular layers of liquid-crystal films," Nature, vol. 355, pp. 152-154, 1992.

[55] T. Stoebe, P. Mach, and C. Huang, "Unusual layer-thinning transition observed near the smectic-A-isotropic transition in free-standing liquid-crystal films," Physical Review Letters, vol. 73, pp. 1384-1387, 1994.

[56] A. Einstein, "Über die von der molekularkinetischen Theorie der Wärme geforderte Bewegung von in ruhenden Flüssigkeiten suspendierten Teilchen," Annalen der Physik, vol. 17, pp. 549-560, 1905.

[57] P. Atkins and J. de Paula, Atkins' Physical Chemistry. Oxford University Press, 2002.

[58] S. Chandrasekhar, "Stochastic problems in physics and astronomy," Reviews of Modern Physics, vol. 15, pp. 1-89, 1943.

[59] M. Cohen and D. Turnbull, "Molecular transport in liquids and glasses," The Journal of Chemical Physics, vol. 31, pp. 1164-1169, 1959.

[60] J. G. Krüger, "Diffusion in thermotropic liquid crystals," Review Section of Physics Letters, vol. 4, pp. 229-269, 1982.

[61] F. Volino and A. Dianoux, "Neutron incoherent scattering law for diffusion in a cosine potential in one dimension: Application to self-diffusion in smectic phases," Molecular Physics, vol. 36, pp. 389-399, 1978. 
[62] F. Volino, A. Dianoux, and A. Heidemann, "Self-diffusion coefficients of TBBA: comparison between neutron and NMR results," Journal de Physique Lettres, vol. 40, pp. 583-586, 1979.

[63] J. Töpler, B. Alefeld, and T. Springer, "Quasielastic neutron scattering to determine self-diffusion constants in liquid p-azoxyanisol," Molecular Crystals and Liquid Crystals, vol. 26, pp. 297-303, 1974.

[64] A. J. Dianoux, F. Volino, A. Heidemann, and H. Hervet, "Neutron quasi-elastic scattering study of translational motions in the smectic H, C and A phases of TBBA," Journal de Physique Lettres, vol. 36, pp. 275-277, 1975.

[65] C. S. Johnson Jr., "Diffusion ordered nuclear magnetic resonance spectroscopy: principles and applications," Progress in Nuclear Magnetic Resonance Spectroscopy, vol. 34, pp. 203-256, 1999.

[66] T. Kawai, A. Kubota, K. Kawamura, H. Tsumatori, and T. Nakashima, "Single molecule fluorescence autocorrelation measurement on anisotropic molecular diffusion in nematic liquid crystal," Thin Solid Films, vol. 516, pp. 2666-2669, 2008.

[67] B. Schulz, D. Täuber, F. Friedriszik, H. Graaf, J. Schuster, and C. von Borczyskowski, "Optical detection of heterogeneous single molecule diffusion in thin liquid crystal films," Physical Chemistry Chemical Physics, vol. 12, pp. 11555-11564, 2010.

[68] B. Schulz, D. Täuber, J. Schuster, T. Baumgärtel, and C. von Borczyskowski, "Influence of mesoscopic structures on single molecule dynamics in thin smectic liquid crystal films," Soft Matter, vol. 7, pp. 7431-7440, 2011.

[69] M. Pumpa and F. Cichos, "Slow single-molecule diffusion in liquid crystals," The Journal of Physical Chemistry B, vol. 116, pp. 14487-14493, 2012.

[70] J. Kirstein, B. Platschek, C. Jung, R. Brown, T. Bein, and C. Bräuchle, "Exploration of nanostructured channel systems with single-molecule probes," Nature Materials, vol. 6, pp. 303-310, 2007.

[71] Y. Sako, S. Minoguchi, and T. Yanagida, "Single-molecule imaging of EGFR signalling on the surface of living cells," Nature Cell Biology, vol. 2, pp. 168-172, 2000.

[72] B. Valeur, Molecular Fluorescence: Principles and Applications. WILEY-VHC, 2001. 
[73] J. R. Lakowicz, Principles of Fluorescence Spectroscopy. Springer, 2006.

[74] E. Brooks Shera, N. K. Seitzinger, L. M. Davis, R. A. Keller, and S. A. Soper, "Detection of single fluorescent molecules," Chemical Physics Letters, vol. 174, pp. 553-557, 1990.

[75] E. Hecht, Optics. Addison-Wesley, 2001.

[76] A. Yildiz, J. N. Forkey, S. A. McKinney, T. Ha, Y. E. Goldman, and P. R. Selvin, "Myosin V walks hand-over-hand: Single fluorophore imaging with 1.5-nm localization," Science, vol. 300, pp. 2061 - 2065, 2003.

[77] U. Kubitscheck, O. Kückmann, T. Kues, and R. Peters, "Imaging and tracking of single GFP molecules in solution,” Biophysical Journal, vol. 78, pp. 2170-2179, 2000.

[78] H. Qian, M. Sheetz, and E. Elson, "Single particle tracking. Analysis of diffusion and flow in two-dimensional systems," Biophysical Journal, vol. 60, pp. 910-921, 1991.

[79] M. J. Saxton, "Single-particle tracking: the distribution of diffusion coefficients," Biophysical Journal, vol. 72, pp. 1744-1753, 1997.

[80] A. J. Leadbetter, J. L. A. Durrant, and M. Rugman, "The density of 4 n-octyl-4-cyanobiphenyl (8CB)," Molecular Crystals and Liquid Crystals, vol. 34, pp. 231-235, 1977.

[81] D. A. Dunmur, M. R. Manterfield, W. H. Miller, and J. K. Dunleavy, "The dielectric and optical properties of the homologous series of cyano-alkyl-biphenyl liquid crystals," Molecular Crystals and Liquid Crystals, vol. 45, pp. 127-144, 1978.

[82] H.-R. Dübal, C. Escher, W. Hemmerling, I. Müller, D. Ohlendorf, and R. Wingen, "Use of liquid-crystal 5-phenylpyrimidine derivates as components of smectic liquid-crystal mixtures," 1990.

[83] B. R. Ratna, R. Shashidhar, G. G. Nair, S. Krishna Prasad, C. Bahr, and G. Heppke, "Evidence of a first-order smectic-A-smectic-C* transition and its approach to tricritical behavior," Physical Review A, vol. 37, pp. 1824-1826, 1988.

[84] J. Thoen, H. Marynissen, and W. V. Dael, "Temperature dependence of the enthalpy and the heat capacity of the liquid-crystal octylcyanobiphenyl (8CB)," Physical Review A, vol. 26, pp. 2886-2905, 1982. 
[85] F. Hardouin, M. F. Achard, G. Sigaud, and H. Gasparoux, "Magnetic anisotropy and polymorphism in liquid crystals," Molecular Crystals and Liquid Crystals, vol. 39, pp. 241-257, 1977.

[86] J. W. Goodby and G. W. Gray, "Smectic F phases exhibited by the N-(4n-alkoxybenzylidene)-4'-n-alkylanilines," Molecular Crystals and Liquid Crystals, vol. 56, pp. 43-49, 1979.

[87] C. Bahr and D. Fliegner, "Behavior of a first-order smectic-A-smectic-C transition in free-standing liquid-crystal films," Physical Review A, vol. 46, pp. 7657-7663, 1992.

[88] H. Tajalli, A. G. Gilani, M. S. Zakerhamidi, and P. Tajalli, "The photophysical properties of Nile red and Nile blue in ordered anisotropic media," Dyes and Pigments, vol. 78, pp. 15-24, 2008.

[89] N. Ghoneim, "Photophysics of Nile red in solution: Steady state spectroscopy," Spectrochimica Acta Part A, vol. 56, p. 1003-1010, 2000.

[90] K. Norrman, A. Ghanbari-Siahkali, and N. B. Larsen, "Studies of spin-coated polymer films," Annual Reports on Progress in Chemistry, vol. 101, pp. 174-201, 2005.

[91] S. Bardon, M. P. Valignat, A. M. Cazabat, W. Stocker, and J. P. Rabe, "Study of liquid crystal prewetting films by atomic force microscopy in tapping mode," Langmuir, vol. 14, pp. 2916-2924, 1998.

[92] K. J. Seu, A. P. Pandey, F. Haque, E. A. Proctor, A. E. Ribbe, and J. S. Hovis, "Effect of surface treatment on diffusion and domain formation in supported lipid bilayers," Biophysical Journal, vol. 92, p. 2445-2450, 2007.

[93] L. Danos, R. Greef, and T. Markvart, "Efficient fluorescence quenching near crystalline silicon from Langmuir-Blodgett dye films," Thin Solid Films, vol. 516, pp. 7251-7255, 2008 .

[94] A. Zywocinski, F. Picano, P. Oswald, and J. C. Géminard, "Edge dislocation in a vertical smectic-A film: Line tension versus temperature and film thickness near the nematic phase," Physical Review E, vol. 62, pp. 8133-8140, 2000.

[95] G. Binnig, C. F. Quate, and C. Gerber, “Atomic force microscope," Physical Review Letters, vol. 56, p. 930-933, 1986. 
[96] R. Garcia and R. Perez, "Dynamic atomic force microscopy methods," Surface Science Reports, vol. 47, pp. 197-301, 2002.

[97] Q. Zhong, D. Inniss, K. Kjoller, and V. B. Elings, "Fractured polymer/silica fiber surface studied by tapping mode atomic force microscopy," Surface Science Letters, vol. 290, pp. L688-L692, 1993.

[98] T. Pompe, A. Fery, and S. Herminghaus, "Imaging liquid structures on inhomogeneous surfaces by scanning force microscopy," Langmuir, vol. 14, p. 2585-2588, 1998.

[99] W. Guo, S. Herminghaus, and C. Bahr, "Controlling smectic focal conic domains by substrate patterning," Langmuir, vol. 24, pp. 8174-8180, 2008.

[100] R. Lucht, C. Bahr, and G. Heppke, "Wetting behavior above the liquid-crystal-isotropic transition in a homologous series," Physical Review E, vol. 62, pp. 2324-2333, 2000.

[101] C. Bahr, "Surface triple points and multiple-layer transitions observed by tuning the surface field at smectic liquid-crystal-water interfaces," Physical Review Letters, vol. 99, p. 057801(4), 2007.

[102] D. W. Berreman, "Optics in stratified and anisotropic media: 4 x 4-matrix formulation," Journal of the Optical Society of America, vol. 62, pp. 502-510, 1972.

[103] S. N. Jasperson and S. E. Schnatterly, "An improved method for high reflectivity ellipsometry based on a new polarization modulation technique," Review of Scientific Instruments, vol. 40, pp. 761-767, 1969.

[104] F. Cichos, C. von Borczyskowski, and M. Orrit, "Power-law intermittency of single emitters," Current Opinion in Colloid and Interface Science, vol. 12, pp. 272-284, 2007.

[105] M. Heidernätsch, "Development of a computer program for simulation and analysis of particle movement in thin liquid films," Master's thesis, TU Chemnitz, 2009.

[106] M. Heidernätsch, M. Bauer, D. Täuber, G. Radons, and C. von Borcyskowski , “An advanced method of tracking temporarily invisible particles in video imaging," diffusionfundamentals.org, vol. 11, p. 111(2), 2009. 
[107] J. Schuster, F. Cichos, and C. von Borczyskowski, "Diffusion in ultrathin liquid films," European Polymer Journal, vol. 40, pp. 993-999, 2004.

[108] I. Kraus, P. Pieranski, E. Demikhov, H. Stegemeyer, and J. Goodby, "Destruction of a first-order smectic-A-smectic-C* phase transition by dimensional crossover in freestanding films," Physical Review E, vol. 48, pp. 1916-1923, 1993.

[109] A. J. Leadbetter, J. C. Frost, J. P. Gaughan, G. W. Gray, and A. Mosley, “The structure of smectic A phases of compounds with cyano end groups," Journal de Physique, vol. 40, pp. 375-380, 1979.

[110] D. Montiel, H. Cang, and H. Yang, "Quantitative characterization of changes in dynamical behavior for single-particle tracking studies," The Journal of Physical Chemistry B, vol. 110, pp. 19763-19770, 2006.

[111] F. Schneider, "Measurement of the viscosity coefficient 3 in free-standing smectic films," Physical Review E, vol. 74, p. 021709(8), 2006.

[112] E. Lauga and T. M. Squires, "Brownian motion near a partial-slip boundary: A local probe of the no-slip condition," Physics of Fluids, vol. 17, p. 103102(16), 2005.

[113] M. Gitterman, "Mean first passage time for anomalous diffusion," Physical Review E, vol. 62, pp. 6065-6070, 2000.

[114] P. G. Saffman and M. Delbrück, "Brownian motion in biological membranes," Proceedings of the National Academy of Sciences, vol. 72, pp. 3111-3113, 1975.

[115] A. Eremin, S. Baumgarten, K. Harth, and R. Stannarius, "Two-dimensional microrheology of freely suspended liquid crystal films," Physical Review Letters, vol. 107, p. 268301(4), 2011.

[116] L. Bocquet and J.-L. Barrat, "Diffusive motion in confined fluids: Mode-coupling results and molecular-dynamics calculations," Europhysics Letters, vol. 31, pp. 455-460, 1995.

[117] Y. Alméras, J.-L. Barrat, and L. Bocquet, "Influence of wetting properties on diffusion in a confined fluid," Journal de Physique IV, vol. 10, pp. 7-27-7-31, 2000. 
[118] B. Martinez-Haya, A. Cuetos, S. Lago, and L. F. Rull, "A novel orientation-dependent potential model for prolate mesogens," The Journal of Chemical Physics, vol. 122, p. 024908(8), 2005.

[119] T. Bellini, N. A. Clark, C. D. Muzny, L. Wu, C. Garland, D. W. Schaefer, and B. J. Oliver, "Phase behavior of the liquid crystal 8CB in a silica aerogel," Physical Review Letters, vol. 69, pp. 788-791, 1992.

[120] G. B. Kasting, C. W. Garland, and K. J. Lushington, "Critical heat capacity of octylcyanobiphenyl (8CB) near the nematic-smectic A transition," Journal de Physique, vol. 41, pp. 879-884, 1980.

[121] R. Guégan, D. Morineau, R. Lefor, W. Béziel, M. Guendouz, L. Noirez, A. Henschel, and P. Huber, "Rich polymorphism of a rod-like liquid crystal (8CB) confined in two types of unidirectional nanopores," The European Physical Journal E, vol. 26, pp. 261273, 2008.

[122] H. Takezoe, M. Hara, S. Ichikawa, and A. Fukuda, "Binary mass diffusion measurements in nematic and smectic liquid crystals by forced Rayleigh scattering," Molecular Crystals and Liquid Crystals, vol. 122, pp. 169-175, 1985.

[123] S. V. Dvinskikh, I. Furo, H. Zimmermann, and A. Maliniak, "Anisotropic self-diffusion in thermotropic liquid crystals studied by ${ }^{1} \mathrm{H}$ and ${ }^{2} \mathrm{H}$ pulse-field-gradient spin-echo NMR," Physical Review E, vol. 65, p. 061701(9), 2002.

[124] H. Kneppe, F. Schneider, and N. K. Sharma, "A comparative study of the viscosity coefficients of some nematic liquid crystals," Berichte der Bunsengesellschaft für physikalische Chemie, vol. 85, pp. 784-789, 1981.

[125] J. A. Forrest and K. Dalnoki-Veress, "The glass transition in thin polymer films," Advances in Colloid and Interface Science, vol. 94, pp. 167-196, 2001.

[126] Y. Pu, H. White, M. H. Rafailovich, J. Sokolov, A. Patel, C. White, W.-L. Wu, V. Zaitsev, and S. A. Schwarz, "Probe diffusion in thin PS free-standing films," Macromolecules, vol. 34, pp. 8518-8522, 2001.

[127] E. K. Hobbie and C. C. Huang, "Thermal-conductivity studies near the smecticA-smectic-C transition in a liquid-crystal compound," Physical Review A, vol. 36, pp. 5459-5462, 1987. 
[128] K. Lammertsma and B. V. Prasad, "Imine - Enamine tautomerism," Journal of the American Chemical Society, vol. 116, pp. 642-650, 1994.

[129] T. Tanaka, E. Sato, Y. Hirokawa, S. Hirotsu, and J. Peetermans, "Critical kinetics of volume phase transition of gels," Physical Review Letters, vol. 55, pp. 2455-2458, 1985.

[130] A. G. Chmielewski and E. Lepakiewicz, "Rheological properties of some biphenyl liquid crystals," Rheologica Acta, vol. 23, pp. 207-210, 1984.

[131] Q. He, W. Yu, Y. Wu, and C. Zhou, "Shear induced phase inversion of dilute smectic liquid crystal/polymer blends," Soft Matter, vol. 8, pp. 2992-3001, 2012.

[132] M. Miesowicz, "The three coefficients of viscosity of anisotropic liquids," Nature, vol. 158, p. $27,1946$.

[133] W. Helfrich, "Conduction-induced alignment of nematic liquid crystals: Basic model and stability considerations," The Journal of Chemical Physics, vol. 51, pp. 4092-4105, 1969.

[134] E. Demikhov, "Surface reconstruction and finite-size effects in smectic free standing films," Molecular Crystals and Liquid Crystals, vol. 265, pp. 403-434, 1995.

[135] E. Lacaze, J. P. Michel, M. Goldmann, M. Gailhanou, M. de Boissieu, and M. Alba, "Bistable nematic and smectic anchoring in the liquid crystal octylcyanobiphenyl (8CB) adsorbed on a $\mathrm{MoS}_{2}$ single crystal," Physical Review E, vol. 69, p. 041705(8), 2004.

[136] N. A. Clark, "Surface memory effects in liquid crystals: Influence of surface composition," Physical Review Letters, vol. 55, pp. 292-296, 1985.

[137] Y. Shi, B. Cull, and S. Kumar, "Morphology and ordering in thin liquid-crystal films on flat glass substrates," Physical Review Letters, vol. 71, pp. 2773-2776, 1993.

[138] J. Tamayo and R. Garcia, "Deformation, contact time, and phase contrast in tapping mode scanning force microscopy," Langmuir, vol. 12, pp. 4430-4435, 1996.

[139] J. P. Cleveland, B. Anczykowski, A. E. Schmid, and V. B. Elings, "Energy dissipation in tapping-mode atomic force microscopy," Applied Physics Letters, vol. 72, pp. 2613$2615,1998$. 
[140] W. W. Scott and B. Bhushan, "Use of phase imaging in atomic force microscopy for measurement of viscoelastic contrast in polymer nanocomposites and molecularly thick lubricant films," Ultramicroscopy, vol. 97, pp. 151-169, 2003.

[141] N. Zhao, G. A. Botton, S. Zhu, and A. Duft, "Microscopic studies on liquid crystal poly(3,3"'-dialkylquaterthiophene) semiconductor," Macromolecules, vol. 37, pp. 8307-9812, 2004.

[142] K. Harth, B. Schulz, C. Bahr, and R. Stannarius, "Atomic force microscopy of menisci of free-standing smectic films," Soft Matter, vol. 7, pp. 7103-7111, 2011.

[143] R. Garcia, J. Tamayo, and A. San Paulo, "Phase contrast and surface energy hysteresis in tapping mode scanning force microsopy," Surface and Interface Analysis, vol. 27, pp. 321-316, 1999.

[144] F. Vandenbrouck, M. P. Valignat, and A. M. Cazabat, "Thin nematic films: Metastability and spinodal dewetting," Physical Review Letters, vol. 82, pp. 2693-2696, 1999.

[145] D. Ausserré and J.-L. Buraud, "Late stage spreading of stratified liquids: Theory," The Journal of Chemical Physics, vol. 134, p. 114706(8), 2011.

[146] T. Jin, G. P. Crawford, R. J. Crawford, S. Zumer, and D. Finotello, "Surface ordering transitions at a liquid crystal-solid interface above the isotropic smectic-A transition," Physical Review Letters, vol. 90, p. 015504(4), 2003.

[147] A. Honciuc, A. W. Harant, and D. K. Schwartz, "Single-molecule observations of surfactant diffusion at the solution-solid interface," Langmuir, vol. 24, pp. 6562-6566, 2008.

[148] B. Lin, J. Yu, and S. A. Rice, "Direct measurements of constrained Brownian motion of an isolated sphere between two walls," Physical Review E, vol. 62, pp. 3909-3919, 2000 .

[149] R. D. Piner, J. Zhu, F. Xu, S. Hong, and C. A. Mirki, “'Dip-pen” nanolithography," Science, vol. 283, pp. 661-663, 1999.

[150] D. Ausserre and M.-P. Valignat, "Wide-field optical imaging of surface nanostructures," Nano Letters, vol. 6, pp. 1384-1388, 2006. 


\section{Publications}

(1) B.Schulz and Ch. Bahr, "Surface structure of ultrathin smectic films on silicon substrates: Pores and islands," Physical Review E, vol. 83, pp. 041710(4), 2011

(2) K. Harth, B. Schulz, Ch. Bahr, and R. Stannarius, "Atomic force microscopy of menisci of free-standing smectic films", Soft Matter, vol. 7, pp. 7103-7111, 2011

(3) A. Sengupta, B. Schulz, E. Ouskova, and Ch. Bahr, "Functionalization of microfluidic devices for investigation of liquid crystal flows", Microfluidics and Nanofluidics, vol. 13, pp. 941-955, 2012

(4) B. Schulz, P. Steffen, and Ch. Bahr "A novel viscoplastic effect and its application in nanolithography" (in preparation) 



\section{Acknowledgments}

$\mathrm{A} \mathrm{PhD}$ thesis would be incomplete without mentioning the people that directly or indirectly supported the successful completion of the work.

First of all, I would particularly like to thank my supervisor Christian Bahr for his guidance through the PhD times. The knowledgeable support and the ever availability for scientific discussion were of greatest help.

My thanks go to Stephan Herminghaus, for giving me the chance to work in his group. The unique creative atmosphere that he establishes together with the depth of thoughts and scientific approaches left a strong impression on me.

I want to thank Jörg Enderlein for being a member of my thesis committee.

I am grateful to have worked with wonderful colleagues from the group of Dynamics of Complex Fluids (here particularly the group of Liquid Crystals) and the group of Droplets, Membranes and Interfaces. It was a great time inside and outside the lab that I will not forget.

The administrative and technical support in the background should never be understated. I want to thank Monica Teuteberg for her great organisational talent and her ever helpfulness in case of problems. For the support in setting up the experiments, I want to thank Udo Krafft and Wolf Keiderling. For keeping the computers running, I owe thanks to Thomas Eggers and Kristian Hantke.

I want to thank Kirsten Harth and Ralf Stannarius for the great and efficient collaboration.

For doing the tedious and indispensable job of proof reading, I want to thank Christian Bahr, Thomas Schulz, and Anupam Sengupta.

It should never be forgotten to thank the people that supplied distraction from science, thereby refreshing the mind, which is essential for finishing a thesis. Not necessarily in order of importance I am thinking of: Anupam, Quentin, Paul, Birte, Marta, Kris, Ciro, Zrinka, Antoine, Martin, Eric, Jeff, and Anne. 
I also want to thank my family for their mental (and financial) support throughout my entire studies. It is fantastic to have people that would always back you up in your life.

Finally, I want to express my gratitude to have Iki at my side. I am happy to be with someone who is supporting me in every single way and makes the sun shine brighter.

"They fly toward grace." Thomas Pynchon 


\section{Curriculum Vitae}

\section{Personal Details}

Name : $\quad$ Benjamin Schulz

Date of birth : $\quad 22.04 .1984$

Place of birth : Karl-Marx-Stadt, Germany

Address : $\quad$ Posthof 2, 37081 Göttingen

Nationality : German

\section{Education}

09/2009 - 03/2013 PhD thesis at the Max Planck Institute for Dynamics and Self-Organization, Göttingen, Germany

Supervisors: Dr. Christian Bahr and Prof. Stephan Herminghaus

$2008-2009$

Diploma thesis, Technical University of Chemnitz, Germany

Supervisors: Dr. Jörg Schuster and Prof. Christian von Borczyskowski

$2007-2008$

ERASMUS studies at the Université Bordeaux 1, France

$2003-2009$

Studies of physics at the Technical University of Chemnitz, Germany 


\section{Erklärung}

Hiermit erkläre ich, dass ich die vorliegende Arbeit selbstständig angefertigt, nicht anderweitig zu Prüfungszwecken vorgelegt und keine anderen als die angegebenen Hilfsmittel verwendet habe. Sämtliche wissentlich verwendete Textausschnitte, Zitate oder Inhalte anderer Verfasser wurden ausdrücklich als solche gekennzeichnet.

Göttingen, im März 2013

Benjamin Schulz 